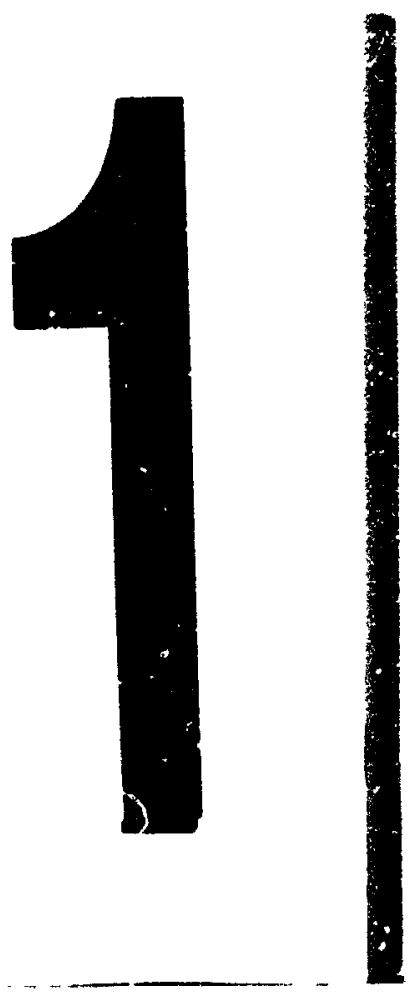

PM-1 31/2" $X 4$ " PHOTOGRAPHIC MICAOCOPY TARGET NBS 1010 ANSL/180 H2 EOUNALENT

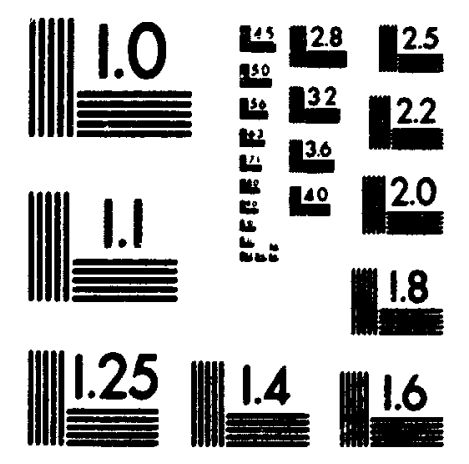

PRECISIONEW RESOLUTON TARGETS

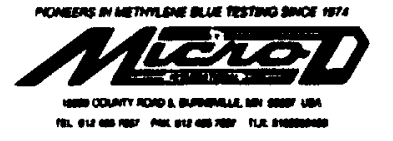


National Library

of Canada

Acquisitions and

Bibliographic Services Branch

395 Wellinglon Streel

Ottawa, Ontario

KIA ON4
Bibliotheque nationale

du Carada

Direction des acquisitions et

des services bibliographiques

395, ne Wellington

Otawa (Ontano)
Vour the Vithe roterence

Our the Notre renterencico

NCTICE

AVIS

The quality of this microform is heavily dependent upon the quality of the original thesis submitted for microfilming. Every effort has been made to ensurz the highest quality of reproduction possible.

If pages are missing, contact the university which granted the degree.

Some pages may have indistinct print especially if the original pages were typed with a poor typewriter ribbon or if the university sent us an inferior photocopy.

Reproduction in fill or in part of this microform is governed by the Canadian Copyright Act, R.S.C. 1970, c. C-30, and subsequent amendments.
La qualité de cette microforme dépend grandement de la qualité de la thèse soumise au microfilmage. Nous avons tout fait pour assurer une qualité supérieure de reproduction.

S'il manque des pages, veuillez communiquer avec l'université qui a conféré le grade.

La qualité d'impression de certaines pages peut laisser à désirer, surtout si les pages originales ont été dactylographiées à l'aide d'un ruban usé ou si l'univarsité nous a fait parvenir une photocopie de qualité inférieure.

La reproduction, même partielle, de cette microforme est soumise à la Loi canadienne sur le droit d'auteur, SRC 1970, c. C-30, et ses amendements subséquents. 


\title{
GATE LEVEL TRANSISTOR SIZING BY NONLINEAR OPTIMIZATION
}

\author{
by
}

\author{
Raymond W. L., Chow, B. Sc.
}

\author{
A thesis submitted to \\ the Faculty of Graduate Studies and Research \\ in partial fulfilment of \\ the requirements for the degree of \\ Master of Science \\ Information and System Science \\ Department of Mathematics and Statistics \\ Carleton University \\ Ottawa, Canada. \\ June 15, 1992 \\ (C) copyright
}

1992, Raymond W. L. Chow 
National Library

of Canada

Acquisitions and

Bibliographic Services Branch

395 Wellington Street

Ollawa. Ontario

K1A ON4
Bıbliotheque nationale

Ju Canada

Direction des acquisitions et

des services bibliographiques

395. rue Wellinglon

Otlawa (Ontario)

KIA ON4

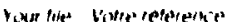

The author has granted an irrevocable non-exclusive licence allowing the National Library of Canada to reproduce, loan, distribute or sell copies of his/her thesis by any means and in any form or format, making this thesis available to interested persons.
L'auteur a accordé une licence irrévocable et non exclusive permettant à la Bibliothèque nationale du Canada de reproduire, prêter, distribuer ou vendre des copies de sa thèse de quelque manière et sous quelque forme que ce soit pour mettre des exemplaires de cette thèse à la disposition des personnes intéressées.

L'auteur conserve la propriété du droit d'auteur qui protège sa thèse. Ni la thèse ni des e'traits substantiels de celle-ci ne doivent être imprimés ou autrement reproduits sans son autorisation. 
The undersigned hereby recommend to the Faculty of Graduate Studies and Research acceptance of the thesis,

"GATE LEVEL TRANSISTOR SIZING

BY

NONLINEAR OPTIMIZATION"

submitted by

Raymond W. L. Chow, B. Sc.

in partial fulfilment of the requirements

for the degree of Master of Science

\section{Joixom}

Chair, Department of Mathematics and Statistics

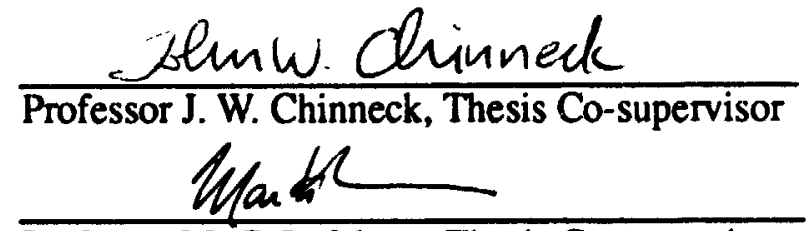

Professor M. C. Lefebvre, Thesis Co-supervisor

Carleton University

June 15, 1992 


\begin{abstract}
Transistor sizing is one of the major steps in designing high performance digital VLSI circuits. In this thesis, a transistor sizing problem is mathematically formulated into a nonlinear optimization problem to study the trade-off between a circuit's delay and the total circuit area. New delay models are used in the experimental set up. New area models are developed based on the information from standard cells and target gates. These new area models are also applied to the experiments to examine their efficiency and their accuracy. A software prototype is developed for the experiments and is tested with several examples covering different structures and sizes of the circuits. With ihis software prototype, a study of the interaction between the time and the area is presented. The study shows that the new delay and area models yield better results than the compared approach, especially for larger circuits.
\end{abstract}




\section{Acknowledgements}

The success of this study is only possible when a healthy environment of resources are available. These resources include a large number of individuals, state of the art equipment, and financial participation from several sources.

First of all, I would like to thank my supervisors, Professor John Chinneck and Professor Martin Lefebvre, who have guided me throughout the project, and I am especially thankful to Professor Martin Lefebvre for introducing the subject to me. Their dedication to my progress has been highly motivational.

I would also like to thank the faculty, staff, and my fellow graduate students of the Department of Electronics and the Department of Mathematics and Statistics for enduring my endless problems. In particular, Richard Griffith, David Skoll, and Roger Shum have been especially helpful.

Finally, I wisli to acknowledge the following sources of financial support: the Department of Electronics and the Department of Mathematics and Statistics, Carleton University, and the Natural Sciences and Engineering Research Council Strategic Grant, under the special Microelectronics Fund. The software implementation and experimentation were run on a network of SUN workstations provided by the Canadian Microelectronics Corporation (C.M.C.). 


\section{Table of Contents}

Acceptance Sheet $\ldots \ldots \ldots \ldots \ldots \ldots \ldots \ldots \ldots \ldots \ldots \ldots$ ii

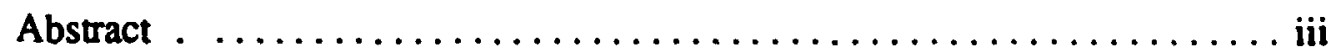

Acknowledgements $\ldots \ldots \ldots \ldots \ldots \ldots \ldots \ldots \ldots \ldots \ldots$ iv

Table of Contents . . ............................ v

List of Tables $\ldots \ldots \ldots \ldots \ldots \ldots \ldots \ldots \ldots \ldots \ldots \ldots$ viii

List of Figures $\ldots \ldots \ldots \ldots \ldots \ldots \ldots \ldots \ldots \ldots \ldots \ldots \ldots \ldots$

List of Algorithms $\ldots \ldots \ldots \ldots \ldots \ldots \ldots \ldots \ldots \ldots \ldots \ldots \ldots \ldots$

Chapter 1: Introduction

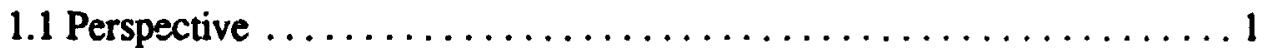

1.2 The General Transistor Sizing Problem ................

1.2.1 The General Nonlinear Optimization problem ......... 2

1.2.2 The Transistor Sizing problem ................

1.2.3 Fixed Cell Library approach $\ldots \ldots \ldots \ldots \ldots \ldots \ldots \ldots \ldots$

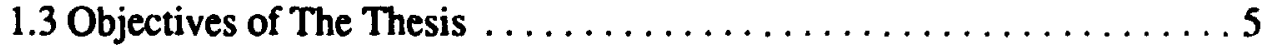

1.4 Organization of The Thesis $\ldots \ldots \ldots \ldots \ldots \ldots \ldots \ldots \ldots$

\section{Chapter 2: Related Work}

2.1 Delay Modeling prior to optimization $\ldots \ldots \ldots \ldots \ldots \ldots 7$

2.1.1 RC Delay Models $\ldots \ldots \ldots \ldots \ldots \ldots \ldots \ldots \ldots \ldots$

2.1.1.1 Direct Mapping Technique $\ldots \ldots \ldots \ldots \ldots \ldots \ldots$

2.1.1.2 The Equivalent Inverter Mapping Technique $\ldots \ldots \ldots 12$

2.1.1.3 Device Equatioris with Waveshape Effects . . . . . . . 15

2.1.2 Piecewise-Linear Timing Delay Model . . . . . . . . . . . . 22

2.1.3 Stepwise Equivalent Conductance Delay model . . . . . . . 24 
2.2 Optimization algorithm chosen prior to delay modeling $\ldots \ldots \ldots 27$

2.2.1 SUMT with Augmented Lagrangian algorithm . . . . . . . . 27

2.2.2 Simplex algorithm . . . . . . . . . . . . . . . . 28

2.2.3 The Simulated Annealing algorithm ............. 28

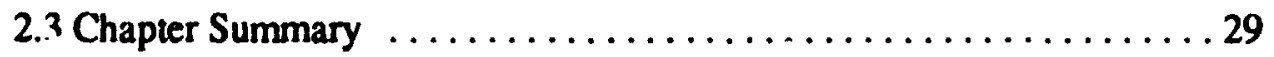

Chapter 3: The Gate Level Transistor Sizing Problem

3.1 Modeling Delay and Area $\ldots \ldots \ldots \ldots \ldots \ldots \ldots \ldots \ldots \ldots \ldots \ldots$

3.1.1 Delay Models . . . . . . . . . . . . . . . . . . 32

3.1 .2 Area Models ............................ 33

3.2 Variable and Parameter Declarations $\ldots \ldots \ldots \ldots \ldots \ldots$

3.3 Problem Statement . . . . . . . . . . . . . . . . . . . 39

3.4 Choosing an Optimization Algorithm $\ldots \ldots \ldots \ldots \ldots \ldots \ldots \ldots \ldots \ldots$

Chapter 4: New Gate Level Transistor Sizing Algorithm

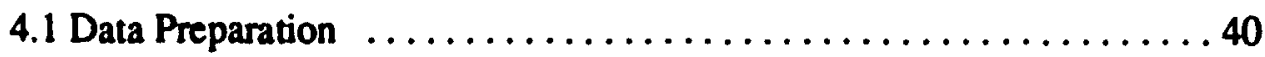

4.1.1 Graph Construction $\ldots \ldots \ldots \ldots \ldots \ldots \ldots \ldots \ldots \ldots \ldots \ldots \ldots \ldots$

4.1.2 Consuructing Delay Equations $\ldots \ldots \ldots \ldots \ldots \ldots \ldots \ldots \ldots 4$

4.2 Critical Path Analysis $\ldots \ldots \ldots \ldots \ldots \ldots \ldots \ldots \ldots \ldots \ldots$

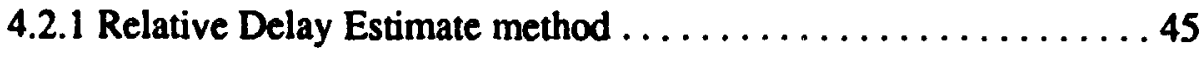

4.2.2 Critical Path Method . . . . . . . . . . . . . . . . . 46

4.3 Gate Size Estimation $\ldots \ldots \ldots \ldots \ldots \ldots \ldots \ldots \ldots \ldots \ldots \ldots \ldots$

4.4 Piecewise Linear and Cubic Approximation of the Area ........50

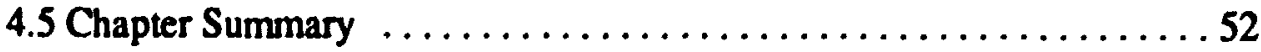

Chapter 5: Optimization

5.1 Formulation of the Nonlinear Programming Problem . . . . . . . 57

5.1 .1 Timing Constraints $\ldots \ldots \ldots \ldots \ldots \ldots \ldots \ldots \ldots \ldots$

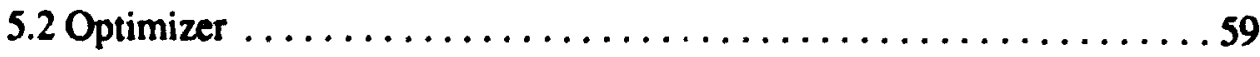


5.2.1 Generalized Reduced Gradient method ............660

5.2 .2 Advantages of the GRG method . . . . . . . . . . . 62

Chapter 6: Implementation and Experimental Results

6.1 The Software Prototype .......................... 63

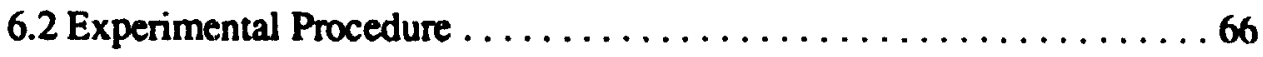

6.2 .1 Experimental Setup ........................66

6.2.2 Optimization process $\ldots \ldots \ldots \ldots \ldots \ldots \ldots \ldots \ldots \ldots .67$

6.2.3 Optimization Results .......................6. 69

6.3 Design Space Exploration \& Fixed Cell Library Approach ........ 73

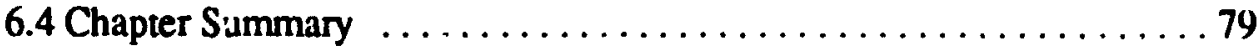

Chapter 7: Conclusions

7.1 Conclusion $\ldots \ldots \ldots \ldots \ldots \ldots \ldots \ldots \ldots \ldots \ldots \ldots \ldots \ldots \ldots \ldots$.

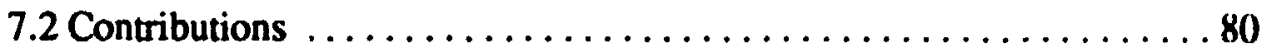

7.3 Recommendations for Future Work $\ldots \ldots \ldots \ldots \ldots \ldots \ldots \ldots 1$

Appendix A. Glossary of Terms and Symbols .................83

Appendix B. User Manual . . . . . . . . . . . . . . . . . 85

Appendix C. Circuit files for the Test Problems and their results .........90)

Appendix D. Sample Fortran subroutines codes ............... 100

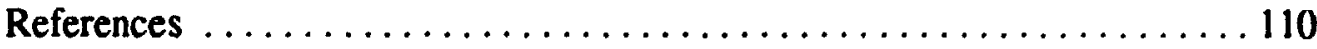




\section{List of Tables}

Table 3.1 The Staircase Area model $\ldots \ldots \ldots \ldots \ldots \ldots \ldots \ldots \ldots$

Table 4.1 Estimate the relative delay for each type of gate $\ldots \ldots \ldots \ldots 47$

Table 4.2 Calculation of event time for Example1 . . . . . . . . . 48

Table 6.1 Values used for calculating the actual area of gates $\ldots \ldots \ldots 67$

Table 6.2 Optimization Results: Gates Information $\ldots \ldots \ldots \ldots \ldots$

Table 6.3 Optimization Results: Nodal Information . . . . . . . . 72 


\section{List of Figures}

Fig.2.1 RC delay models (a) MOS transistor (b) distributed RC delay model. 8

Fig.2.2 Geometrical interpretation of generalized gradient. . . . . . . 10

Fig.2.3 Geometrical calculation of $\operatorname{Nr}\left(\partial_{\varepsilon} \varphi(x)\right) \ldots \ldots \ldots \ldots \ldots \ldots$

Fig.2.4 (a) NAND with $\mathrm{N}$ inputs, (b) Equivalent Inverter Circuit. . . . . 12

Fig.2.5 (a) RC model for Fig.2.4a, (b) RC model for Fig.2.4b. . . . . . 13

Fig.2.6 Delay mapping as amplifier and resistor. $\ldots \ldots \ldots \ldots \ldots \ldots$

Fig.2.7 Circuit for determining drive curves. $\ldots \ldots \ldots \ldots \ldots \ldots \ldots$

Fig.2.8 Drive curves for an inverter model. . . . . . . . . . . 17

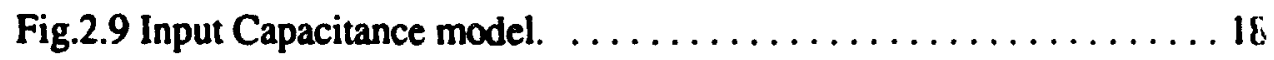

Fig. 2.10 Expected input capacitance. . . . . . . . . . . . 19

Fig.2.11 Inner loop minimization. $\ldots \ldots \ldots \ldots \ldots \ldots \ldots \ldots \ldots \ldots$ 20

Fig.2.12 Outer loop maximization. $\ldots \ldots \ldots \ldots \ldots \ldots \ldots \ldots \ldots \ldots \ldots$

Fig.2.13 Two-segment piecewise linear approximation. . . . . . . . 23

Fig.2.14 Equivalent Conductance of a MOS transistor and

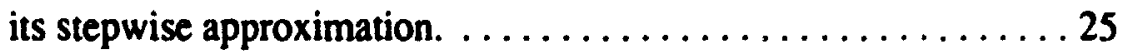

Fig.2.15 Electrical model for a MOS transistor... . . . . . . . . 26

Fig.2.16 Construction of electrical model from a network. . . . . . . 26

Fig.3.1 Effect on area by increasing the size of an inverter

in standard cell design. $\ldots \ldots \ldots \ldots \ldots \ldots \ldots \ldots \ldots \ldots$

Fig.3.2 The staircase area model. $\ldots \ldots \ldots \ldots \ldots \ldots \ldots \ldots \ldots \ldots$

Fig. $3.3 \mathrm{~A}$ linear area model. $\ldots \ldots \ldots \ldots \ldots \ldots \ldots \ldots \ldots \ldots \ldots$

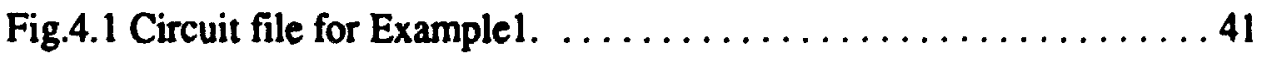

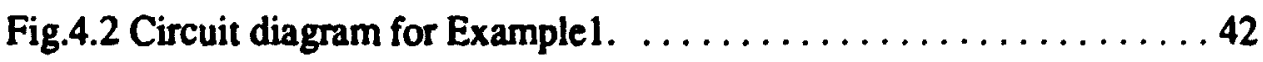

Fig.4.3 DAG - Network model for Example1. . . . . . . . . . 42

Fig.4.4 Table of information for Examplel. . . . . . . . . . . 44 
Fig.4.5 AOA network for Examplel showing the relative delay estimates. . 48 Fig.4.6 Activity slack calculation for the network of Example1. . . . . 50 Fig.4.7 Estimates of the gate sizes are shown on edges for Example1. . . . 52 Fig.4.8 Piecewise Approximation of an Inverter. . . . . . . . . . 54 Fig.4.9 A diagram to explain the approximation of the staircase function. . 55 Fig.5.1 Illustration of setting up network constraints. . . . . . . . 58

Fig.5.2 Set of constraints for Examplel. . . . . . . . . . . . . 59

Fig.6.1 Flow Chart for the Transistor Sizing software prototype. . . . . . . 65 Fig.6.2 Network diagram for Example 1 with gate and node information. . 70 Fig. 6.3 Desired trade off between area and speed. . . . . . . . . . 74 Fig.6.4 Trade-off between the total area and the speed for Example 1. . . 75 Fig.6.5 Trade-off between the total area and the speed for Example 2. . . . 76 Fig.6.6 Trade-off between the total area and the speed for Example 3. . . . 77 Fig.6.7 Trade-off between the total area and the speed for Example 4. . . 78 


\section{List of Algorithms}

Algorithm 4.1 General algorithm for solving the Gate Level TSP. . . . . . 40

Algorithm 4.2 Procedure to construct the DAG format table. . . . . . . 43

Algorithm 4.3 Procedure to construct the delay equations. . . . . . . 45

Algorithm 4.4 Algorithm to estimate the initial gate sizes. . . . . . . . 57 


\section{Chapter 1}

\section{Introduction}

\section{l.1 Perspective}

In recent years, CMOS technology has become the major technology for digital VLSI design because it provides high performance at lower power, and scales well to small feature sizes. High performance means that a circuit runs at a very high speed but it still achieves either a low power consumption or a small circuit area or both. Thus, in order to obtain a high performance digital CMOS VLSI designed circuit, making trade-offs among power, speed and area is often required. Designers have traditionally used tools such as circuitlogic simulators and critical path analysis tools to adjust transistor sizes in order to satisfy the design specifications. This iterative process relies on manual adjustments to the sizes followed by re-analysis of the circuit until the design meets the specifications. While adequate for small circuits, this repetitive evaluation of the circuit responses is time consuming for anything larger. Moreover, convergence towards an optimum solution is difficult to achieve. For these reasons, automated assistance in transistor sizing is very much needed. In this thesis, the transistor sizing problem is formulated mathematically and an efficient algorithm is applied to find the optimum transistor sizes.

\subsection{The General Transistor Sizing Problem}

Transistor Sizing is a nonlinear optimization problem. A brief discussion of nonlinear optimization follows. 


\subsubsection{The General Nonlinear Optimization problem}

A general nonlinear optimization problem is described as follows:

$$
\begin{array}{ll}
\min f(x) & \\
\text { s.t. } & \\
& c_{i}(x)=0, i=1, \ldots, m \\
& c_{j}(x) \geq 0, j=m+1, \ldots, p \\
& l_{k} \leq x_{k} \leq u_{k}, k=1, \ldots, n .
\end{array}
$$

where $\mathbf{x}$ is the vector of variables and $f$ is a possibly nonlinear function of $\mathbf{x}$, called $t^{\prime}$ :e objective function of the problem. $c_{i}(x)$ and $c_{j}(x)$ are equality and inequality constraints respectively. Constraints with " $\leq$ " sign can be simply converted to " $Z$ " by multiplying by a factor of $-1 . l_{k}$ and $u_{k}$ are the lower and upper bounds for the variable $x_{k}$. The problem described above has $m$ equality constraints and $(p-m)$ inequality constraints. Together with the upper and lower bounds on all $n$ variables, the goal of the problem is to find the value of $\mathbf{x}$ which minimizes the objective function $f(\mathbf{x})$.

The Objective Function and Constraints - At least one of the objective function or constraints is nonlinear in a nonlinear optimization problem. The structures of both the set of constraints ard the objective function determine the type of algorithm that can be applied to solve the problem. For example, quadratic programming can be applied if the objective function is quadratic and all constraints are linear functions of the design variables. If every term in the objective function and the constraints contains only one single variable, and the set of constraints forms a convex set, then separable programming can be applied. Note that a set $X$ 
is said to be convex if for any points $\mathrm{x}_{1}$ and $\mathrm{x}_{2}$ in $\mathrm{X}$, the line segment joining these two points is entirely contained in $X$.

Bounds - In real world applications, design variables usually bave known upper and lower bounds. Mathematical programming treats these bounds as extra constraints on the problem. A lower bound is converted into a linear constraint of the form:

$$
x-b \geq 0
$$

where $\mathbf{b}$ is the value of the bound. For an upper bound, the linear constraint is

$$
b-x \geq 0
$$

\subsubsection{The Transistor Sizing problem}

A number of researchers $[1,3,4,5,8,9,11,16,17]$ have published results of various studies in this area. In general, the objective of these studies is focused on minimizing either the tral area or the total power consumption of a given circuit subject to a timing restriction on the overall propagation delay through the circuit. The general Transistor Sizing problem is formulated as an optimization problem as follows:

$\min f$

s.t.

$$
\sum_{i} T_{i} \leq T_{\max }, \forall p a t h s
$$

where $f$ is the objective function (i.e. the total area or the total power consumption of the circuit), $T_{i}$ is the delay of circuit element $i$ along any path from a primary input to a primary output and $T_{\max }$ is the user-specified maximum allowable delay. 
Transistor Sizing can be divided into two sub-problems: Delay Modeling and Optimization Strategies. These two sub-problems often have an interactive effect on each other. Delay models can be further subdivided into two main categories: transistor-level models and gate-level models. These two models imply different strategies for design and also affect the complexity of the formulated problem. In transistor-level models, the design variables are the trancistor sizes. A change in any of these variables means resizing a particular transistor. On the other hand, the design variables for gate-level models are the scaling factors associated with each iogic gate. Hence, any changes in thesc scaling factors imply a change to the size of the gate and a resizing of all transistors within that gate according to the new scaling factor. In terms of the complexity of the corresponding mathematical problem, problems at the transistor-level have a larger number of variables when compared with the gate-level problem, hence, the optimization problem is larger at the transistor-level.

\subsubsection{Fixed Cell Library approach}

In this thesis, another sizing approach called the Fixed Cell Library approach is compared to the new algorithm. Under this approach, NAND type and NOR type gates have a fixed size, and the inverter is the only type having a different set of sizes. Since each gate is associated with a fixed size, the delay for the gates can be easily determined with the equations described in Chapter 3. Thus, with a given circuit, gate sizes are assigned according to their types and the maximum delay is determined. Inverters always start with the minimum sizes. The maximum delay through the circuit can only be adjusted by using different sizes of inverters. The results are either infeissible and no further improvement can be made, or the timing constraint on the circuit is met. In either case, the total area of the circuit is calculated along with the maximum propagation delay of the circuit. Since this approach does not involve any optimization process, its advantages are 
simplicity and speed in estimating the circuit area and the circuit propagation delay.

\subsection{Objectives of The Thesis}

There are two objectives for this thesis. The first is to show that the new delay models [15] and area models used in this thesis are useful in practical circuit design. The new delay models are taken as an input to this thesis. They are modeled at the gate level incorporating important information like the input svitching time and the capacitive load of a gate. New area models are developed in this thesis. Given that standard cells have a fixed ceil height, the area occupied by a gate depends heavily on the standard cell height and so:ne other gate parameters, as shown later. These new delay and area models are used in the optimization process, and the results are compared with the results generated by the Fixed Cell Library approach in order to demonstrate the practical use of these novel methods.

The second objective of this thesis is to design an algorithm which can provide a good feasible initial solution as a starting point for any optimizer, including the one used in this thesis, LSGRG. This initial solution speeds up the optimization process and prevents stalling of the optimizer at a local infeasibility when a feasible solution actually exists.

\subsection{Organization of The Thesis}

The remainder of the thesis is organized as follows. A study of the related work on transistor sizing is presented in Chapter 2. Chapter 3 defines the transistor sizing problem addressed in this thesis and gives a detailed description of the corresponding mathematical problem. The new delay and area models will also be 
discussed. The algorithm to generate a feasible initial solution is described in Chapter 4. Chapter 5 presents the theory (GRG method) behind the optimizer, LSGRG, used in this thesis. The experimental results are presented and analyzed in Chapter 6. Chapter 7 provides conclusions and suggestions for future research. 


\section{Chapter 2 Related Work}

Numerous different delay models and optimization strategies have been applied to the transistor sizing problem; this chapter surveys the relevant literature.

The modeling and optimization approaches are so interdependent that they are impossible to separate. For this reason, authors approach the problem in one of two ways: either they develop a delay model and then select an optimization technique which can handle those models, or they start with an optimization technique and develop delay models within the limitations of the optimizer.

Section 2.1 reviews work in which the delay modeling precedes the choice of the optimizer. Three types of delay models are covered: RC delay models, Piecewise Linear Timing delay models and Stepwise Equivalent Conductance delay models. Section 2.2 reviews work in which the choice of the optimizer guided the development of the delay models. Three optimization algorithms are covered: SUMT with Augmented Lagrangian, Simplex LP and Simulated Annealing.

\subsection{Delay Modeling prior to optimization}

\subsection{RC Delay Models}

In a RC delay model, each MOS transistor is modeled by a capacitor ard a switched linear resistor. The capacitor is connected from the gate to the ground and the switched linear resistor is connected from the drain to the source. This resistor is controlled by a gate to source voltage, $V_{G S}$.

Within the RC delay model, there are different techniques to model the delay through the gates. Three different techniques are presented here: the direct 
mapping technique, the equivalent inverter mapping technique and the technique which uses the device equations with waveshape effects.

\subsubsection{Direct Mapping Technique}

Delay Model This technique is applied at the transistor-level [1]. The design variables are the transistor sizes. Fig.2.1a shows a $\mathrm{RC}$ delay model for a MOS transistor and Fig.2.1b shows the delay computation of a distributed RC delay model.

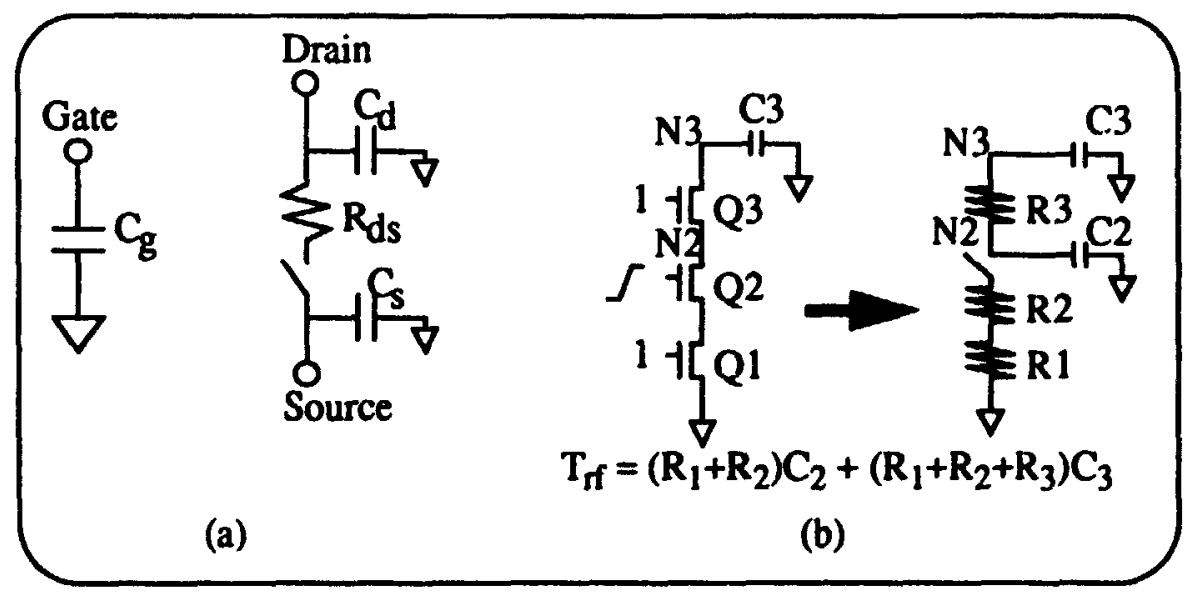

Fig.2.1 RC delay models (a) MOS transistor (b) distributed RC delay mociel.

In Fig.2.1b, $Q$ 's are the nMOS transistors where $Q 1$ and $Q 3$ are always $O N$, and $Q 2$ has a rising input signal. $T_{r f}$ is the discharge time for the NFET pull-down network 1 .The delay equations require the equivalent $R$ 's and C's in the distributed RC model, and these values are determined using the direct mapping technique.

Let $\mathbf{N}$ be a node and $\mathrm{C}_{\mathbf{N}}$ be the corresponding node capacitance. $\mathrm{C}_{\mathbf{N}}$ is estimated according to the number of fan-out gates and the type of node that node $N$ is

1. The model also holds for the PFET pull-up networks. 
linked with. For R's, two types of resistances that must be considered for the delay model: the channel resistance of a transistor $Q, R_{Q}$ and the resistance from a node $\mathbf{N}$ to the power rail, $\mathbf{R}_{\mathrm{N}-\mathrm{P}}$ Once these values are estimated, the time delay of a digital circuit can be computed as the sum of the times to charge/discharge a chain of pull-up/pull-down networks. Since each logic gate consists of a pull-up and a pulldown network only, this implies that the computation for the delay of each logic gate is needed.

Assume that a logic gate has only one output node. Let $T_{r f}^{0}$ be the discharge time for transistor $Q$ in a pull-down network when other transistors on the discharging path to OUT are ON. Let $T_{h}^{N} / T_{l}^{N}$ be the time reference when the signal at the node $\mathbf{N}$ goes high/low. Then,

$$
T_{l}^{O U T}=\max \left\{T_{h}^{N N_{i}}+T_{r f}^{Q_{i}}\right\}
$$

for all $Q_{i}$ belong to the pull-down network. Similarly, $T_{h}^{o v T}$ can be computed by considering the pull-up network. Once the output node of a gate is specified, the transistors where drains/sources are connected to that node are searched to form the pull-up/pull-down networks, those transistors are then grouped into a gate in order to compute the delays.

Optimization Strategy The optimization strategy applied in this case is the Phase I - Phase II Method of Feasible Directions (MFD). Notice that the delay equation is defined as the maximum of all possible delay paths between a primary input and a primary output. Hence, it is a nondifferentiable function. In order to apply the MFD algorithm to the problem, the concept of Generalized Gradient is introduced.

Let $\varphi(x)=\max \left(s_{i}(x)\right), \mathrm{i}=1, \ldots, \mathrm{m}$ be the circuit delay, while $\mathrm{g}_{\mathrm{i}}(\mathrm{x})$ represents the path delay. The generalized gradient is used to compute the descent directions for the function $\varphi(x)$. Its formal definition is

$$
\partial \varphi(x)=\operatorname{co}\left\{\nabla_{g_{i}}(x) \mid i \in I(x)\right\}
$$

where co denotes the convex hull of the vectors $\nabla_{g_{j}}(x)$, i.e., the set defined by 


$$
\begin{aligned}
& \sum_{i \in T(x)} \mu_{i} \nabla g_{i}(x)=0 \\
& \sum_{i \in T(x)} \mu_{i}=1, \mu_{i} \geq 0, i \in I(x)
\end{aligned}
$$

and $I(x)=\left\{i \mid g_{i}(x)=\varphi(x)\right\}$. When $I(x)$ has only one element, the generalized gradient is just a standard gradient. However, if it has more than one element, $\partial g(x)$ is a set as shown in Fig.2.2.

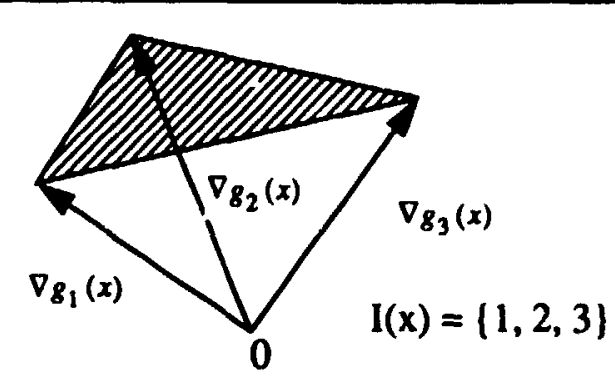

Eig. 2.2 Geometrical interpretation of generalized gradient.

The MFD algorithm uses the idea of the generalized gradient. Let

$$
I_{\varepsilon}(x)=\left\{i \mid\left(\varphi(x)-g_{i}(x)\right) \leq \varepsilon\right\}
$$

be the $\varepsilon$-active set, where $\varepsilon$ is a small positive number. To compute the descent directions for $\varphi(x)$, the set

$$
\partial_{\varepsilon} \varphi(x)=: o_{j \in I_{\varepsilon}(x)}\left\{\nabla g_{j}(x)\right\}
$$

is used. The MFD algorithm then uses the shortest vector in the set, $\partial_{e} \varphi(x)$, to compute the search direction, $h_{\mathcal{E}}(x)$, i.e. $h_{\varepsilon}(x)=-\operatorname{Nr}\left(\partial_{\varepsilon} \varphi(x)\right)$, where $N r$ denotes the nearest vector to the origin or the shortest vector in $\partial_{e} \varphi(x)$ as shown in Fig.2.3. 


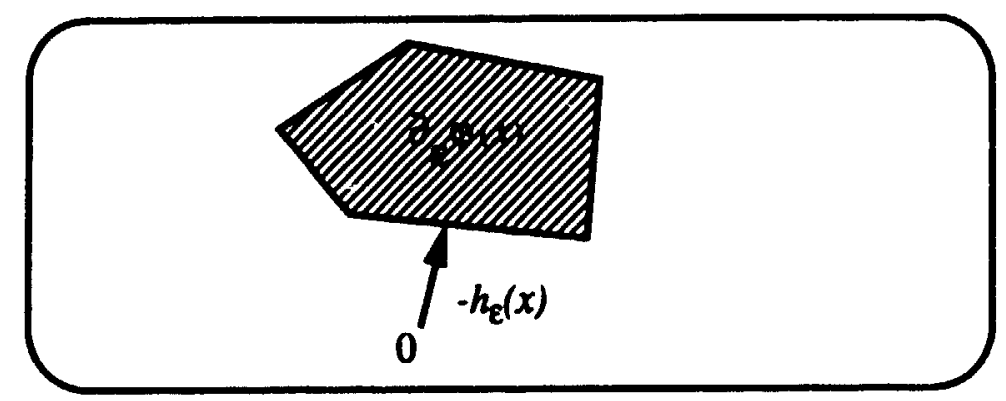

Eig. 2.3 Geometrical calculation of $N r\left(\partial_{2} \varphi(x)\right)$

In order to apply the MFD algorithm, the $\varepsilon$-active delay paths and their derivatives with respect to the design parameters are required. It is equivalent to compute the sensitivities of the $\varepsilon$-active delay paths in circuit design. This is done by defining and identifying different types of transistors and by calculating their derivatives with the information from the RC delay models. Since the information is extracted from existing models, the process of calculating the derivatives is computationally inexpensive. Once these derivative formulae are obtained, MFD can be applied to solve the problem. Phase I of MFD tries to locate a point in the feasible region, while Phase II tries to find an optimum point within the feasible region without leaving it. In both phases, a point $x$ is given and the search direction is computed based on the gradients of the cost and of some of the constraints. A step along the search direction is computed so that the decrease in the cost and the constraint functions is optimized. This iterative process stops when the step size of the search direction vector is small enough.

Interdependency The delay model is a nondifferentiable function and the feasibility of the initial point has not been considered. This implies that the optimization algorithm must find for itself a feasible initial point. The Method of Feasible Directions is one of the best choices. Other algorithms which do not rely on a feasible initial point are hindered by the structure of this model. For example, the Quadratic Programming (QP) algorithm requires that the model is in quadratic 
form and is continuously differentiable.

\subsubsection{The Equivalent Inverter Mapping Technique}

Delay Model This technique maps a CMOS circuit into an equivalent inverter circuit [12]. Since complex gates can be treated as combinations of NAND and NOR type gates, attention is paid to mapping the NAND type and NOR type gates only.

Consider a NAND circuit with $\mathrm{N}$ input signals as shown in Fig.2.4a. The circuit is mapped to an equivalent inverter circuit as shown in Fig. $2.4 \mathrm{~b}$ such that both circuits have equal first-order time constants.

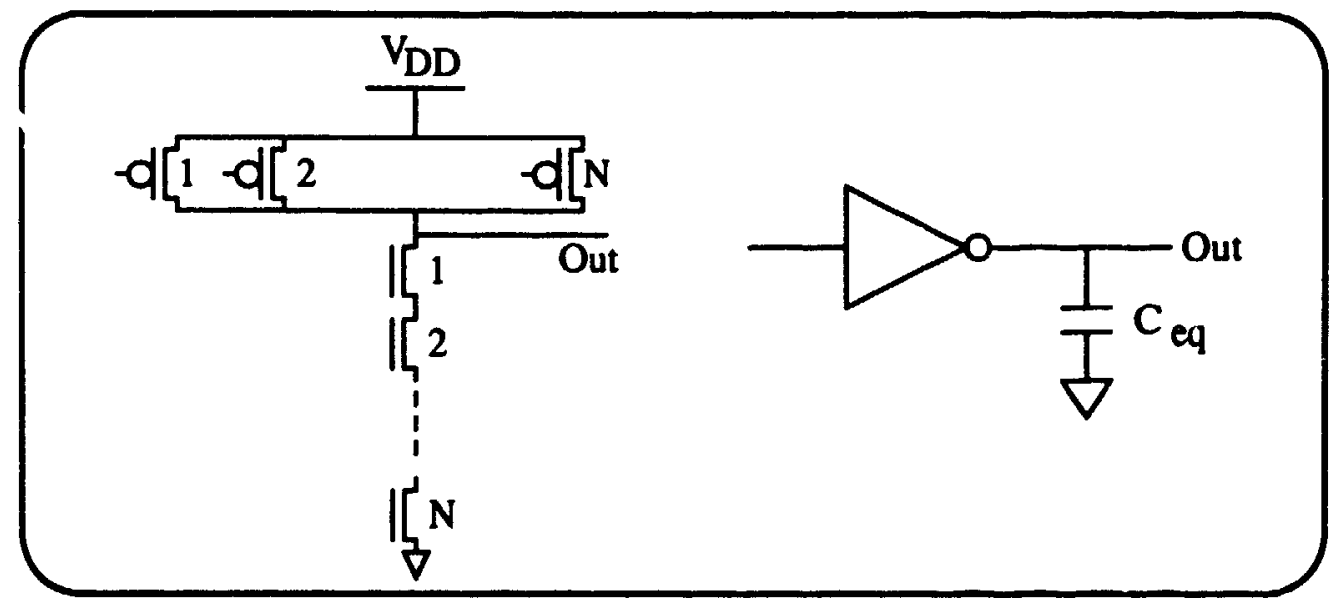

Fig,2,4 (a) NAND with N inputs, (b) Equivalent Inverter Circuit.

Fig. 2.5a and Fig. 2.5b show the RC models for the n-portion of the NAND circuit and the equivalent inverter circuits respectively. 


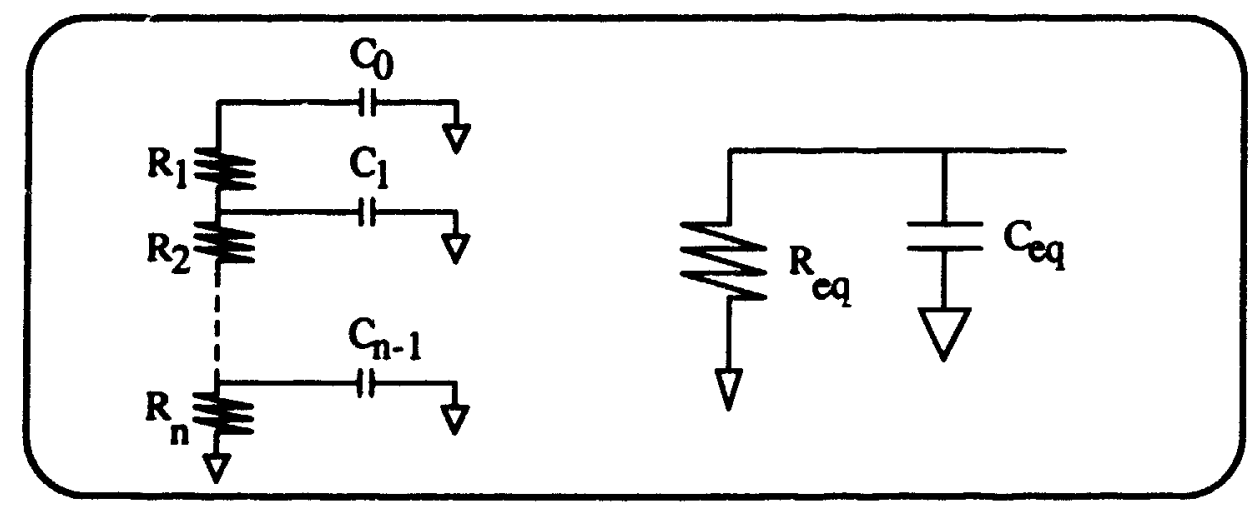

Eig, 2.5 (a) RC model for Fig, 2.4a, (b) RC model for Fig, 2,4b

The first-order moment of the impulse response (the Elmore time constant) of the circuit in Fig.2.5a is

$$
T_{c}=\sum_{i=0}^{N-1} \sum_{j=i+1}^{N} R_{j} C_{i}
$$

where $R_{j}$ is the equivalent resistance for transistor $T_{j}$ and $C_{i}$ is the equivalent capacitance for transistor $T_{j}$. Next, find an inverter circuit with a correct width which can represent an equivalent resistance value, $R_{\text {eq }}$, and a correct capacitance load to the circuit.

Width Consideration For NAND type gates, the n-type transistors are connected in series. Hence, all the $\mathrm{N}$-channel devices will be in the conducting state when voltage drops between the diffusion regions, except one, say device $K$, which will switch from high to low or vice versa. In either case, its average current-driving capability is only half of the other devices. Thus, its effective transistor size is only half of the size of the others. Moreover, if the signal is switching from high to low. the path is blocked beyond the device which is switching. Therefore, only the transistors up to the one which is switching are considered. Thus, the equivalent tran- 
sistor sizes are determined by the following equations:

$$
\begin{aligned}
& W_{p e q}=W_{p K} \\
& \frac{1}{W_{n e q}}=\frac{1}{W_{n K}}+\sum_{i=1, i \neq K}^{M} \frac{1}{2 W_{n i}}
\end{aligned}
$$

where $M=N$ if transistor $T_{K}$ is switching $H I G H$, and $M=K-1$ if switching LOW. Capacitance Mapping In order to preserve the first-order time constants, the sizes of the transistors, except the one which is switching, are made twice as big, or the equivalent resistances are reduced by half:

$$
R_{e q} C_{e q}=\left(\frac{R_{1}}{2}+\ldots+R_{K}+\ldots+\frac{R_{N}}{2}\right) C 0+\ldots+\left(R_{K}+\ldots+\frac{R_{N}}{2}\right) C_{K-1}+\ldots+\frac{R_{N}}{2} C_{N-1}
$$

Since transistor width is inversely proportional to its resistance, Eq. 2.10 can be rewritten as

$$
\frac{C_{e q}}{W_{c q}}=\left(\frac{1}{2 W_{1}}+\ldots+\frac{1}{W_{K}}+\ldots+\frac{1}{2 W_{N}}\right) C 0+\ldots+\left(\frac{1}{W_{K}}+\ldots+\frac{1}{2 W_{N}}\right) C_{K-1}+\ldots+\frac{C_{N-1}}{2 W_{N}}
$$

where $W_{\text {eq }}$ is either $W_{\text {neq }}$ or $W_{\text {peq }}$ depending on whether the circuit is a NAND or NOR gate. The capacitance, $C_{i}$, consists of diffusion capacitances and gate capacitances and is expressed as a complex equation [12]. Hence, the extracted equivalent mapping capacitance, $\mathrm{C}_{\mathrm{eq}}$, is the sum of $\mathrm{C}_{\text {neq }}$ and $\mathrm{C}_{\text {peq, }}$, which is also listed in [12]. Thus, an equivalent resistance and capacitance are found and therefore, the delay is found. The equivalent sizes and capacitances for NOR type gates can be obtained similarly by reversing the suffixes $n$ - and $p$ - in equations 2.8 and 2.9 .

For complex gates, the equivalent $\mathbf{N}$ - and P-channel widths and capacitances are obtained from the routines used for NAND and NOR gates respectively. The last step is to find the path of the complex gate, and an algorithm is given with an assumption that no redundancy exists in the circuit. This algorithm is beyond the scope of this section, details can be found in reference [12]. Once the path is 
determined, the equivalent sizes and capacitances are calculated.

\subsubsection{Device Equations with Waveshape Effects}

Delay Model This is another technique based on a RC delay model [9] in which the effective capacitance is now expressed as a function of the shape of the waveform. Since the falling outputs are highly sensitive to the input slope for the nMOS, particularly when the input is slow, the pull-down is saturated for a significant portion of the transition and causes the inverter to behave like an amplifier. During this time, the inverter is highly sensitive to the input waveform and consequently the resistive model breaks down. For the purpose of illustration, inverters with rising input/falling output nMOS gates are chosen for analysis. The macromodel equations for the opposite transition and for CMOS are similar.

Qutput Waveform The study begins by looking at the inverter's response to different input waveform slopes at different regions of operation. The resistive model works well for a fast input response. For slow inputs, however, the inverter is modeled as an amplifier when the pull-down is saturated, and as a resistor tied to $\mathrm{V}_{\mathrm{OL}}$ when the pull-down is in its linear region. As the input transition becomes faster, the inverter spends proportionately less time as an amplifier and more as a resistor. The mapping of the inverter to an amplifier and resistor is shown in Fig.2.6. 


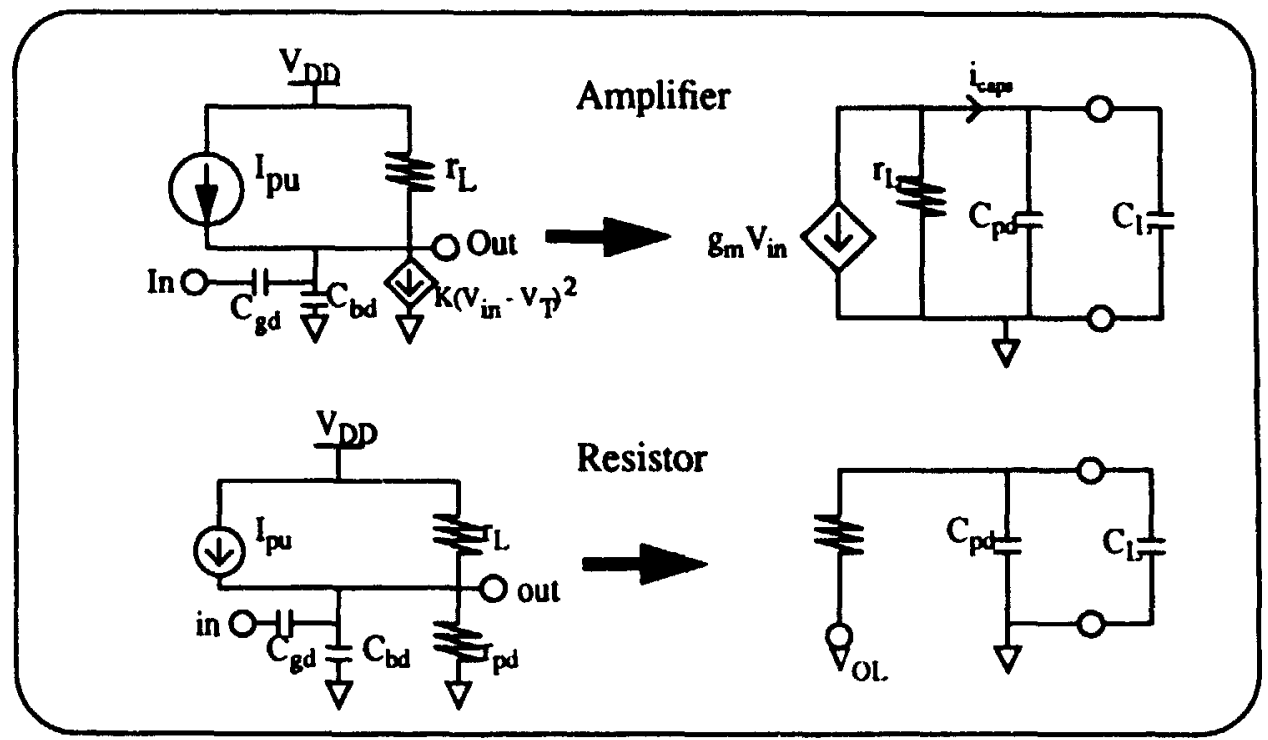

Fig.2.6 Delay mapping as amplifier and resistor.

It starts with an amplifier model when the input voltage reaches $\mathrm{V}_{\mathrm{IL}}$, it then changes to a resistor model as the pull-down transistor firmly enters its linear iegion. For continuity of $\mathrm{V}_{\text {OUT }}$ and $\mathrm{i}_{\mathrm{OUT}}$ when the models are changing, the resistor model is used when $V_{O L} \geq r_{p d} * i_{\text {caps }}+V_{\text {OUT }}$.

Fig. 2.7 shows the circuit for determining drive curves and Fig.2.8 presents the model's drive curves.

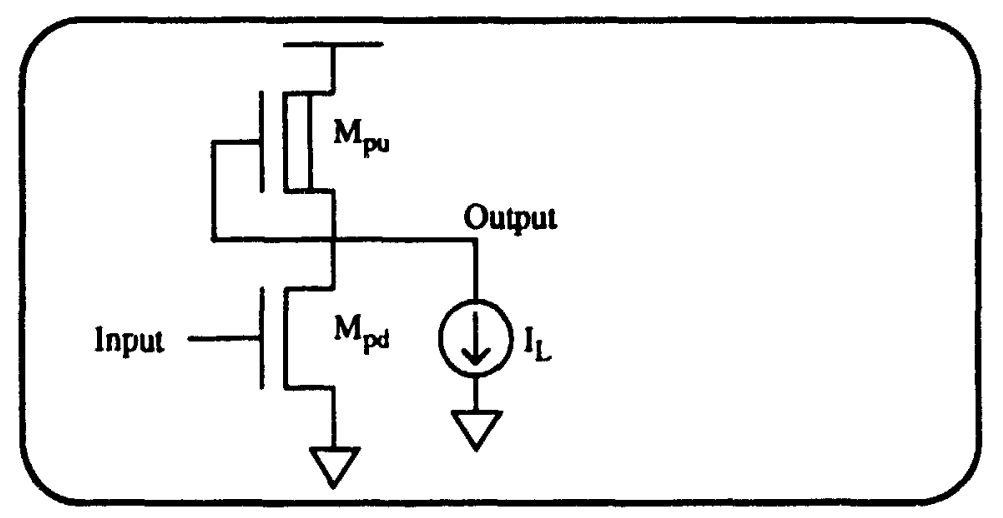

Eig.2.7 Circuit for determining drive curves. 
Notice that the dc transfer curve is flat outside of the range of $V_{\text {in }}$ in $\left(V_{I L}\right.$, $V_{I H}$ ), which implies that the gate will not respond until a rising input has reached $\mathrm{V}_{\mathrm{IL}}$, or until a falling input has dropped to $\mathrm{V}_{\mathrm{IH}}$. This feature provides an immunity to noise for the input signal.

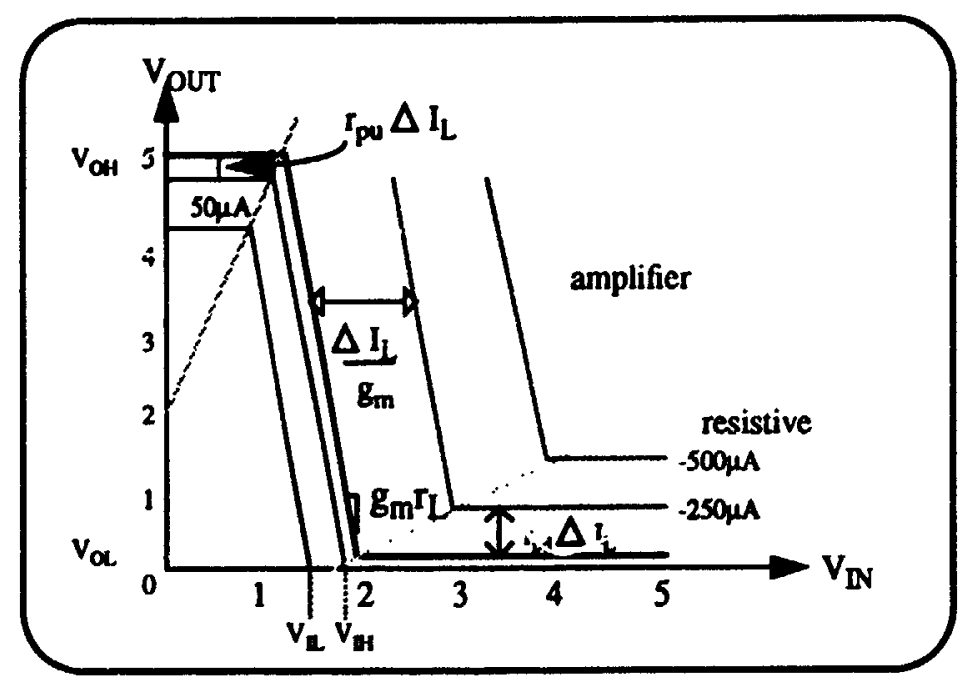

Eig.2.8 Drive curves for an inverter model

Furthermore, resistive behaviour is obviously shown by the horizontal lines on the left and right sides of the figure display. The amplifier mode is represented by the slanted lines in the center of the drive curves. The output waveform is therefore entirely determined by the input waveform and the output slope being proportional to the input slope. To calculate the model's parameters such as $\mathrm{g}_{\mathrm{m}}$ and $\mathrm{r}_{\mathrm{L}}$, expressions are derived analytically for the form of the macromodel equations, then curve fit to observed data to solve for the parameters in the equations.

Input Capacitance Gate delay depends on the input capacitances of the gates that it drives. An inverter has the input capacitance as shown in Fig.2.9. 


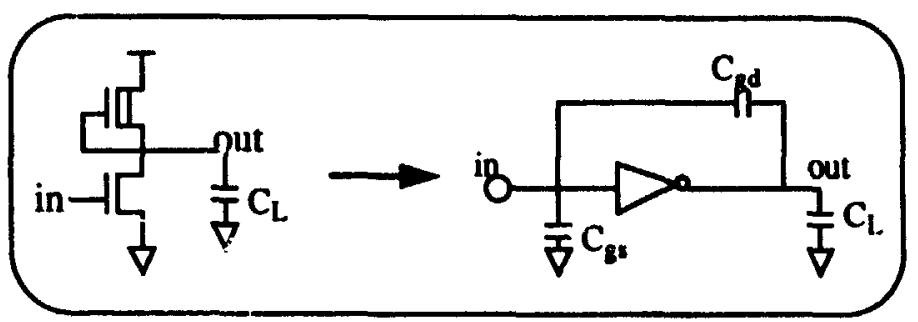

\section{Eig.2.2 Input Capacitance model.}

The input capacitance consists of a gate-to-drain, $\mathrm{C}_{\mathrm{gd}}$, and a gate-to-source, $\mathrm{C}_{\mathrm{gs}}$, capacitances. During the input transition, the input capacitance will be changed due to the input-to-output coupling capacitance, $\mathrm{C}_{\mathbf{g d}}$.

Consider a moderate speed rising input transition. At the early stage (that is, before the input voltage, $V_{i n}$, reaches, $\left.V_{I L}\right)$, the input capacitance is simply $C_{g s}$ $+C_{g d}$. As $V_{\text {in }}$ passes $V_{I L}$, output begins to pull low. Consequently, the driver must supply more current to charge $C_{g d}$ in order to maintain the output voltage. Thus, the effective input capacitance has increased, which is also known as the Miller effect [ 21$]$. For very fast input transitions, the output will not move until after the input has fully switched. Thus, there will not be any Miller capacitance during transition. As the speed of the input transitions slow down, the output switching time overlaps more with the input switching time and more Miller capacitance can be seen. Eventually, all of the output's switching time overlaps with the input's and $\mathrm{C}_{\mathrm{SWin}}{ }^{1}$ reaches a plateau. Thr expected behaviours of the effective input capacitances $\mathrm{C}_{\mathrm{BEin}}{ }^{2}$ and $\mathrm{C}_{\mathrm{SWin}}$ are shown in Fig.2.10.

1. It is a capacitance measured during the output switching period.

2. A capacitance before the output starts responding. 


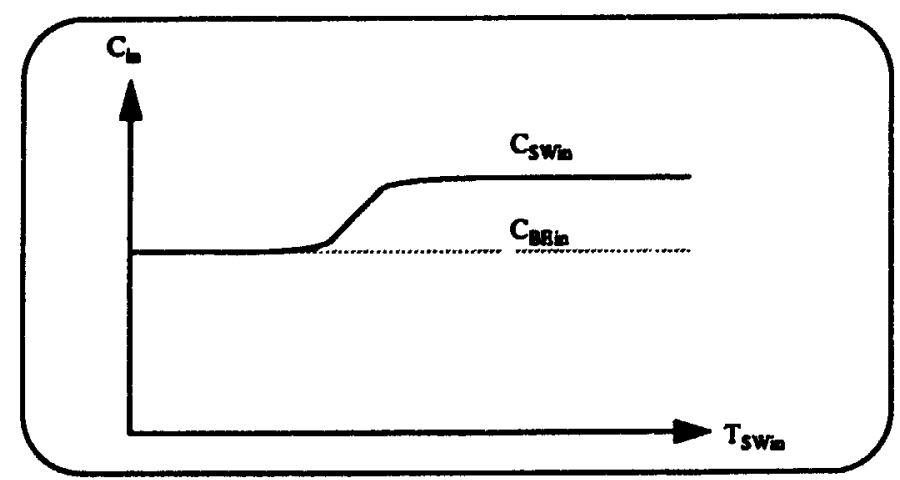

Eig.2.10 Expected input capacitance.

Again, the macromodel equations are obtained through curve fitting ${ }^{1}$.

Optimization Strategy The optimization algorithm applied in this case is the Lagrangian algorithm. It uses a divide-and-conquer strategy where the problem is partitioned into many smaller sub-proble ' $\mathbf{~ s . ~}$

The objective function is the pow: consumption of the circuit, a function of the form: $P=\sum_{i=1}^{n} P_{i}$, where $P_{i}$ is the power consumption of cell $i$. The constraints have two varieties: delay specifications and transistor size design rules. The delay of a signal path is a nonlinear function of the transistor sizes, while the transistor sizes design rules are just simple linear and box constraints. Since the problem involves a large number of design parameters, the theory of duality is used. The approach can be thought as a two-loop process. The first (inner) loop is to move to and remain on the lower bound of the feasible region $R$. It begins with a feasible initial point, $X$ ' and performs the minimization on the Lagrangian function in order to reach the lower bound of $R$, which is described as:

$$
\min \mathrm{L}(\mathbf{x}, \mu)=\{\mathrm{P}(\mathbf{x})+\mu \mathrm{g}(\mathbf{x})\}
$$

s.t.

$x \in X$, with $\mu$ fixed.

1. For complex gates and other details, please refer to reference [9]. 
where $X$ is a set of valid transistor sizes, $L$ is the l-agrangian function and $P(x)$ and $\mathbf{g}(\mathbf{x})$ are the objective and constraint functions respectively. This process is graphically described in Fig.2.11

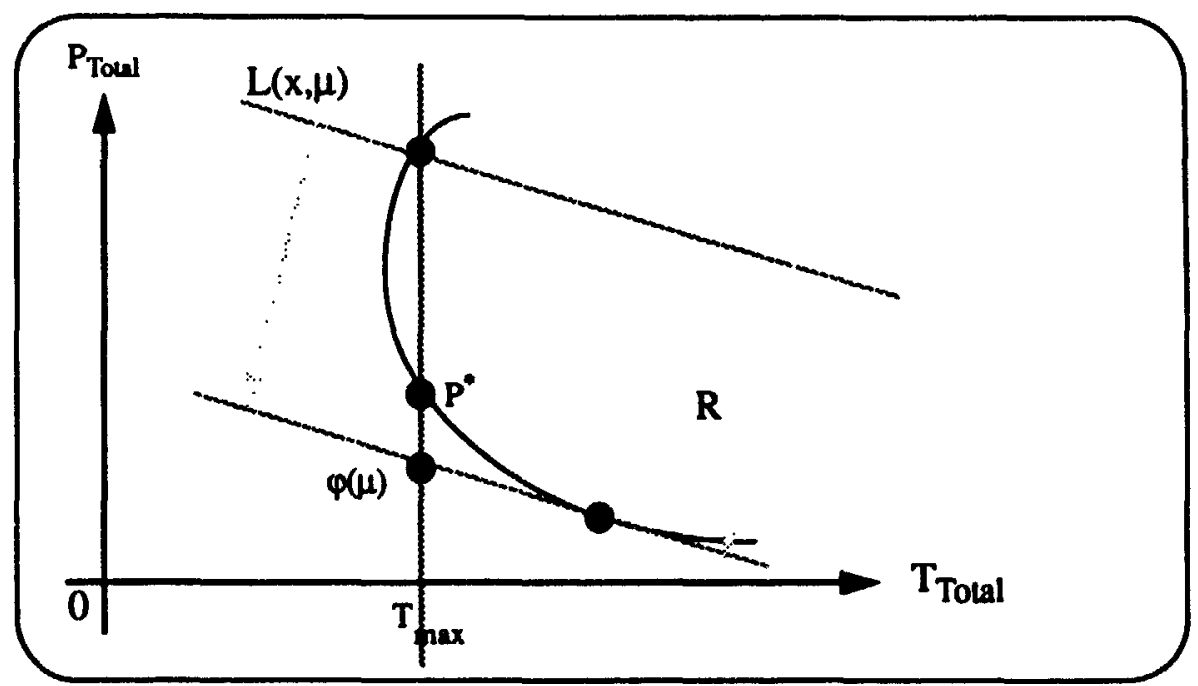

Eig.2.11 Inner loop minimization.

$\varphi(\mu)$ is the minimum intercept of the Lagrangian function $L(x, \mu)$. Since the circuit's power and delay functions are composed of nearly independent contributions from each logic cell, the technique of divide-and-conquer is applied to the minimization process, i.e. minimizing the Lagrangian of each cell in succession. The interactions among cells are taken care of by propagation using a relaxation technique. It iterates the minimization of the entire circuit three times and therefore, the minimization is partitioned over many small vector spaces, leading to small computation times.

The second (outer) loop applies different Lagrange multipliers to the inner loop's minimization in order to find the optimum, which is equivalent to walking along the lower boundary to the optimum point. According to Fig.2.11, the value $\varphi(\mu)$ moves up and down with different Lagrange multipliers. In order to meet the 
optimum point $\left(0, \mathrm{P}^{*}\right)$, the value has to go up. This leads to a maximization in the outer loop over different Lagrange multipliers. This maximization process is described as:

$\max \varphi(\mu)$

s.t. $\quad \mu \geq 0$.

The outer loop is graphically described in Fig. 2.12.

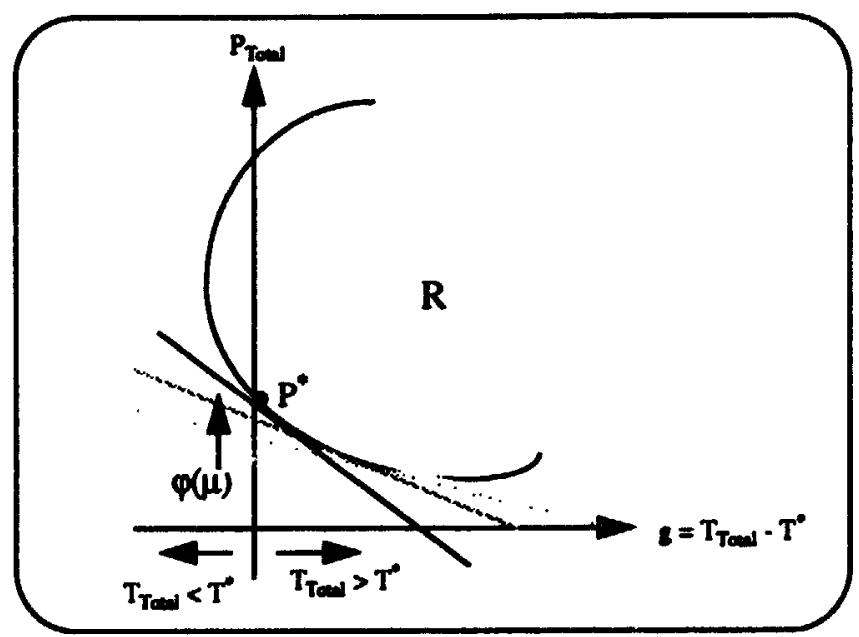

Eig.2.120uter loop maximization.

Thus, the outer loop maximization yields the Lagrange multiplier $\mu$ at the optimum, while the inner loop minimization provides the optimum transistor sizes assignments.

Interdependency Nonlinear delay models are used in this problem. Moreover, the objective and the constraint functions are weakly separable in terms of the design parameters. This is a general nonlinear optimization problem. By converting the original (primal) problem into a dual problem, it is much easier to solve in this case, and it also exploits the separability of the power and delay functions, enabling a divide-and-conquer strategy. Partitioning the problem speeds up the optimization process. 


\subsubsection{Piecewise-Linear Timing Delay Model}

Delay Model This modeling technique [12] characterizes the timing response of some typically and frequently appeared circuit activities. The nonlinear transient waveform is approximated by a simple two-segment piecewise linear waveform. It requires two timing parameters: $\Delta T_{1}$ and $\Delta T_{2}$, which represent the propagation delay and the rise/fall delay respectively.

In general, there are two types of state transitions exhibited at each node of a digital CMOS circuit: either rising or falling between $V_{D D}$ and $G N D$. The transition waveform is asymmetric to the logic threshold voltage, $V_{m}=V_{D D} / 2$, because of its exponential tail. To approximate this nonlinear transition, a two-segment piecewise linear approximation is used. The first segment characterizes the propagation delay defined by $\Delta T_{1}=t_{s o}-t_{s i}$, where $t_{\text {so }}$ and $t_{s i}$ are the times when the input and the output reach the logic threshold voltage, $v_{m}$, respectively. The second segment starts from $t_{s o}$ and ends at $t_{s s}$ at which $V_{\text {out }}$ reaches the steadystate $V_{D D}$ or GND. The output $V_{\text {out }}$ changes very little before the input $V_{\text {in }}$ crosses the logic threshold $V_{m}$, and the effect of the input waveform shape mostly comes from the portion after $V_{\text {in }}$ crosses $V_{m}$. Thus, the rise/fall time delay of the second segment, $\Delta T_{2}=t_{s s}-t_{s o}$, could be used to model the effect of the input waveform shape on the output timing delay when it is driving some other gates. The model is graphically shown in Fig.2.13. 


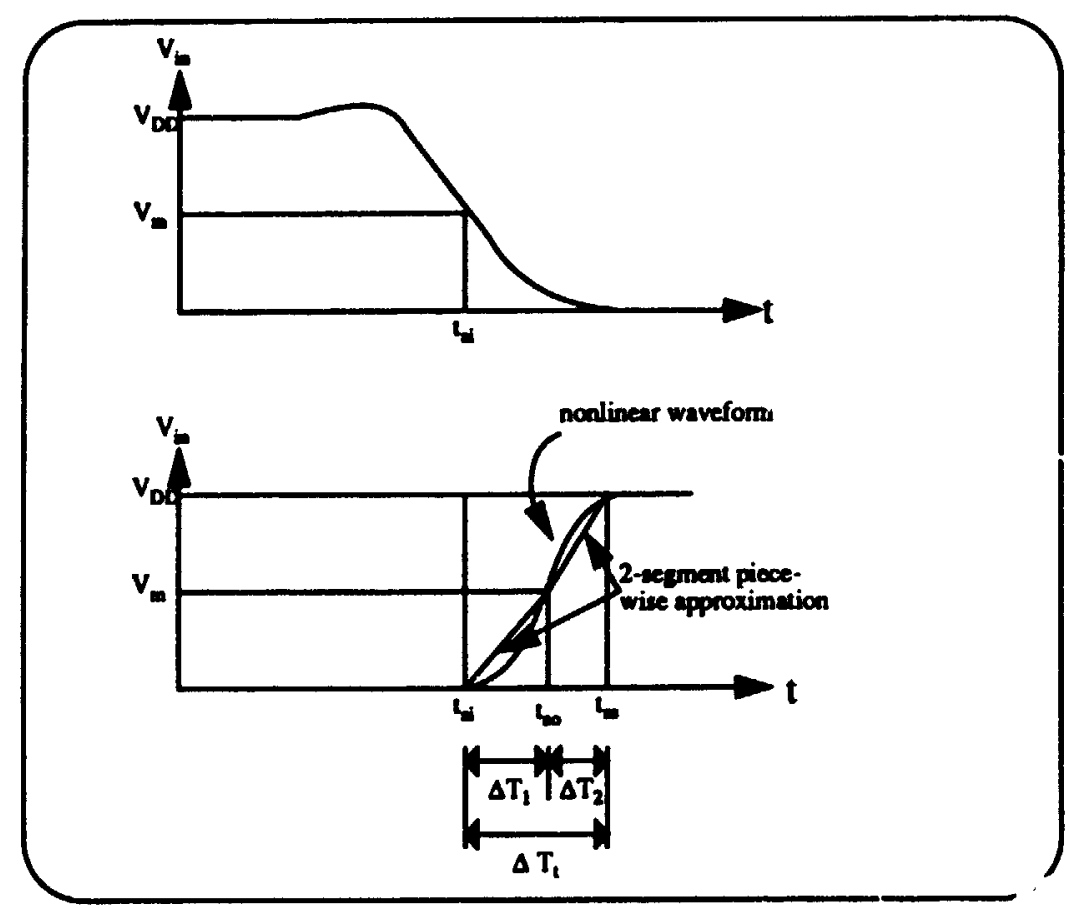

Fig.2.13 Two-segment piecewise linear approximaticel.

The propagation delay, $\Delta \mathrm{T}_{1}$, the rise/fall delay, $\Delta \mathrm{T}_{2}$, and the transition time, $\Delta T_{t}\left(=\Delta T_{1}+\Delta T_{2}\right)$ of the output waveform are determined by parameters such as the transistor and the node capacitances and the circuitry involved in the charging or discharging path of the output node. Some of these parameters involve complicated nonlinear structures, while the others are voltage-dependent, making it difficult to determine these parameters accurately, so an empirical model is used. By circuit simulations or direct measurements, the timing delays for different possible combinations of circuit structures are precharacterized and are stored in a table. The table is then compressed to its minimum size by eliminating any redundancy of data. Redundancy here means different circuit topologies having similar branch patterns. Since most digital circuits are built by interconnecting standard logic gates, such as inverters, NAND gates, NOR gates and some frequently used 
"standard cells", circuits with different circuit topologies might have identical branch patterns for some controlling inputs. Finally, the minimum set of data is mapped into simple linear formulas. This mapping process is done by minimizing the error between the data and the simple linear formulas with unknown coefficients. The set of coefficients obtained corresponds to the minimum approximation error achieved.

Optimization Strategy This is actually a successive library look-up procedure to determine the timing response of a digital circuit. The computational efficiency is solely determined by the procedure to recognize the branch pattern and refer to the correct location in the library.

Interdependency Simple linear delay n:odels are generated by this piecewise linear modeling technique. Although there exist optimization algorithms which can be applied, a library look-up strategy is used. The elimination of an optimization algoritnm helps maintain the accuracy of the delay models and reduces the time for numerical computation. However, unless a huge library is constructed, it is practically impossible to have every kind of sub-circuit stored in the library. Furthermore, a huge library may cause lengthy look-up times which is the major procedure for the method.

\subsubsection{Stepwise Equivalent Conductance Delay model}

Delay Model This technique approximates the equivalent conductance [13] to construct the delay model. At any time point $t$, the equivalent conductance of a MOS transistor, $G(t)$, is defined as the ratio of $I_{D S}$ and $V_{D S}$ :

$$
G(t)=\left[\begin{array}{cc}
\beta \times\left.\left(2\left(V_{G S}-V_{t h}\right)-V_{D S}\right)\right|_{t} & ,\left(V_{G S}-V_{t h}\right) \geq V_{D S} \geq 0 \\
\beta \times\left.\frac{\left(V_{G S}-V_{t h}\right)^{2}}{V_{D S}}\right|_{t} & , v_{D S} \geq V_{G S}-V_{t h} \geq 0 \\
0 & , V_{G S}<V_{t h}
\end{array}\right]
$$


Since this is the only nonlinear element in the MOS transistor model, an approximation by a stepwise function is proposed. During the transition, at each time point, the conductance is calculated according to Eq. 2.12, assuming that these conductances will remain constant for the next time step. Thus, only linear elements are involved in the calculation for the next time step. Fig.2.14 shows the stepwise approximation for the transition.

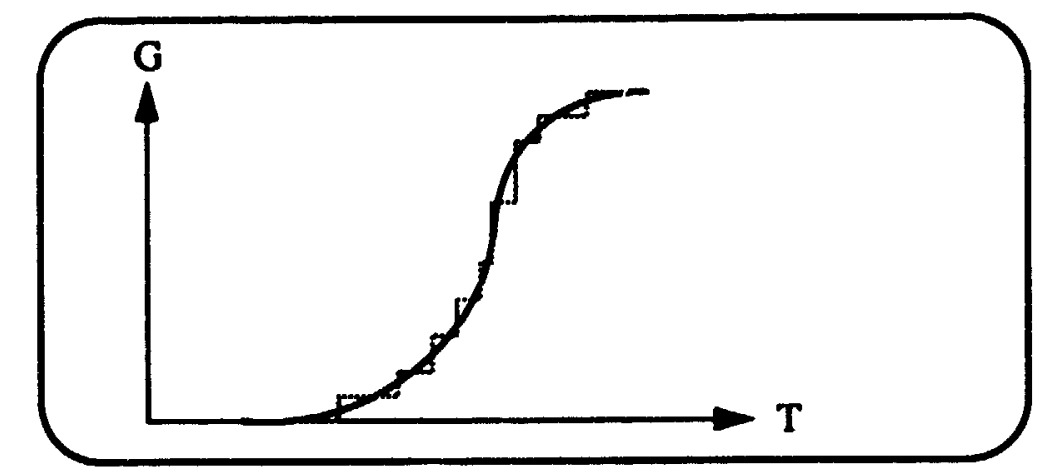

Eig.2.14 Equivalent Conductance of a MOS transistor and its stepwise approximation.

Nodal Analysis In general, nodal analysis is required only for nodes which are the sources or drains of the transistors but not $V_{D D}$ or GND. Consider a gate as shown in Fig.2.16a whose output is initially at 5V. In this case only the pull-down network is considered. The nMOS transistors in the network are replaced by their equivalent electrical models as shown in Fig.2.15. 


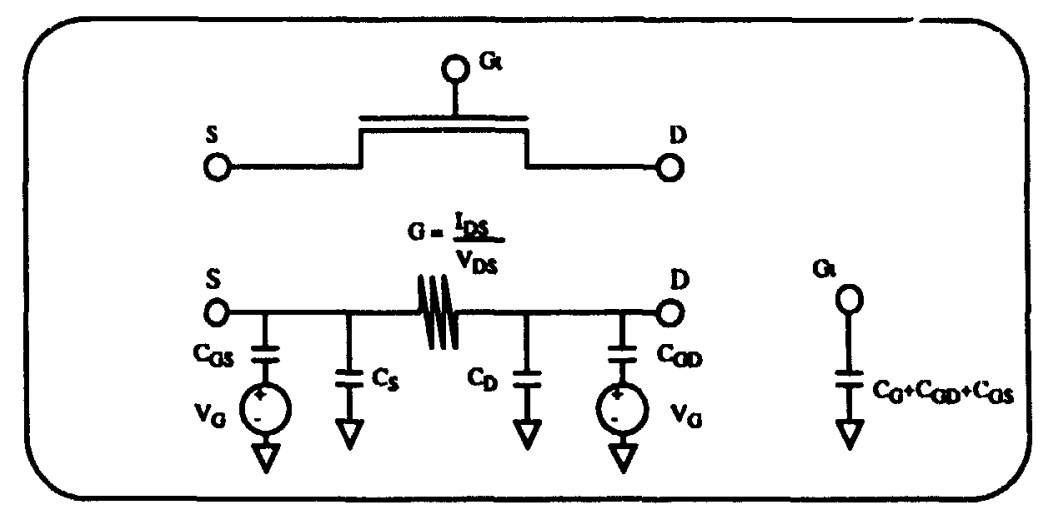

Fig. 2.15 Electrical model for a MOS transistor.

The part of the circuit which contains all the branches connected to nodes (1) and (2) will look like the electrical model in Fig.2.16b. The nodal equation involving the current node capacitances and voltages is derived and the voltage and the equivalent conductance for the next time step is estimated.

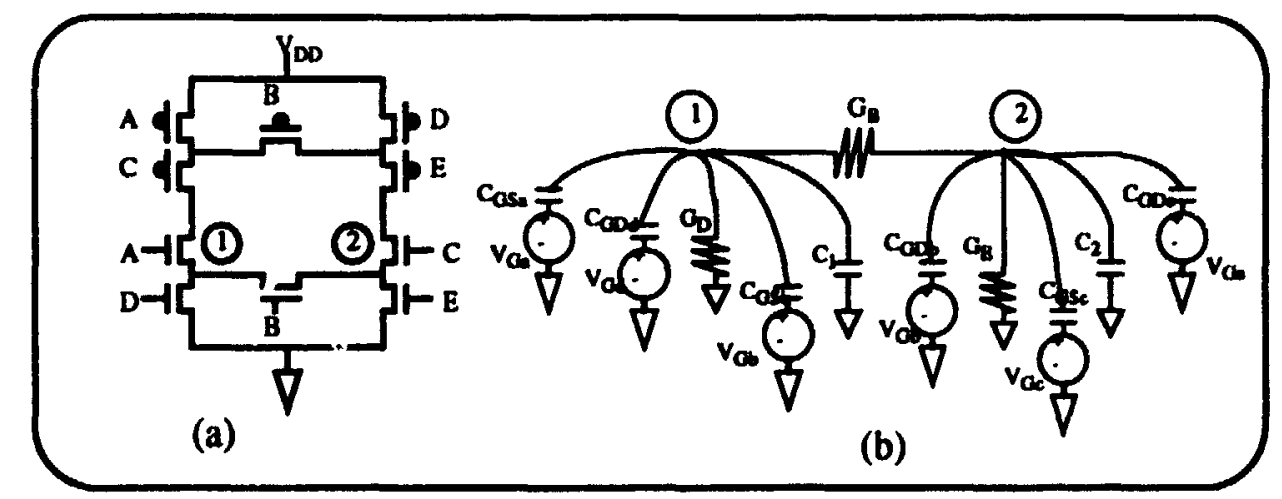

Fig.2.16 Construction of electrical model from a network.

Time Step Variable time steps are used to achieve a balance between speed and accuracy. They are adjusted according to the changing rate of the transistor's conductances. For $\mathrm{RC}$ circuits, $\mathrm{C}$ is time invariant. The changing rate of $\mathrm{R}$ is governed 
by both the changing rate of inputs and its own value (the smaller $R$, the larger its changing rate).

\subsection{Optimization algorithm chosen prior to delay modeling}

The effect of delay modeling on the choice of the optimization strategies was seen in the last section. This section discusses the interrelationship in the opposite way. If a designer chooses an optimization algorithm because of its robustness or efficiency, the delay models will have certain limitations.

A brief description is given for each optimization algorithm discussed in this section, and is followed by a short discussion of the appropriate delay models.

\subsubsection{SUMT with Augmented Lagrangian algorithm}

Optimization algorithm The Sequential Unconstrained Minimization Technique (SUMT) is a general optimization technique. It converts a constrained minimization problem into a sequence of unconstrained sub-problems. This technique combines the Lagrangian function with a penalty function to form the augmented Lagrangian function, $L_{A}(x, \lambda, \rho)$ :

$$
L_{A}(x, \lambda, \rho)=f(x)+\sum_{i}^{m}\left\{\lambda_{i} g_{i}(x)+p h\left(g_{i}(x)\right)\right\}
$$

where $f(x)$ is the objective function, $\lambda_{i}$ 's are the Lagrange multipliers, $g_{i}(x)$ 's are the constraints, $\rho$ is the penalty factor and $h\left(g_{i}(x)\right)$ is the penalty function on the constraints. Since the problem becomes unconstrained, simpler gradient and line search algorithms can be applied. This is an iterative process which requires a given initial point, although the given point does not necessarily to be feasible. The direction with the steepest descent is found and a line search algorithm determines the step size in this direction. This process stops when the step size found by the 
line search is sufficiently small.

Delay Model The delay models allowed under this optimization strategy have more freedom. This is because the algorithm solves a lot of general nonlinear optimization problems, and the delay equations mostly have nonlinear structures. A minor effect from this strategy is that the models should be continuously differentiable, otherwise, complicated numerical algorithm(s) are needed to find the gradient, which decreases the efficiency of the algorithm.

The use of the augmented Lagrangian algorithm has been successfully demonstrated by Marple [5].

\subsubsection{Simplex algorithm}

Optimization Strategy The Simplex method is a well-known algorithm for linear programming problems. It guarantees the final solution is the global optimum (if one exists) and is efficient even for large-scale problems.

Delay Model Delay in digital CMOS circuits has nonlinear relationship among different parameters, but the use of the Simplex restricts the delay models to linear form, so, an approximation of the nonlinear functions is needed. These new linear approximater delay models lose accuracy compared with the original models. Using the simple and fast Simplex algorithm means accepting an approximate solution (if one exists) because of the approximated delay models. The Simplex algorithm has been used for transistor sizing by Berkelaar [11].

\subsubsection{The Simulated Annealing algorithm}

Optimization Strategy The Simulated annealing algorithm has become popular in the area of circuit optimization. It is based on an analogy to the heat treating of metal. The algorithm starts with an initial point (not necessarily feasible, if using a penalty function) at a high "temperature" $T$, which represents the permitted degree of randomness of the solution process. Working along a path, the 
algorithm randomly chooses a cell size between the lower and upper size limits, an determines whether this is an improvement or not.

Let $\mathrm{C}$ be the cost function of the problem (either the area or power consumption of the circuit), if

$$
\Delta C=C(\text { new config })-C(\text { old config })<0
$$

then the new configuration is an improvement an" " kept for next iteration. However, if $\Delta C \geq 0$, then there still exists a probability $e^{-\frac{\lambda}{T}}$ of accepting the new configuration. The "temperature" (randomness) of the process is controlled by a prespecified "cooling" rate, $\mathrm{r}(\mathrm{r}<1)$ such that, for the $\mathrm{k}^{\text {th }}$ iteration, $T_{k}=r \times T_{k-1}$ (e.g. $\mathrm{r}$ $=0.1$ ). The process repeats a specified number of times in order to obtain a stable and optimal solution.

Delay Model Notice that this new optimization strategy is independent of the delay models applied. It does not involve any numerical optimization routines and its iterative procedure contains only the computation of random sizes, the cost function and the probability. The simplicity and delay models independency are the attractive features of this algorithm. However, optimality cannot be assured because of the heuristic structure of the algorithm. Furthermore, long running time for this, algorithm may be needed to achieve a stable and globally optimal solution.

The Simulated annealing algorithm has been used for transistor sizing by Chang [3] for a cell-based transistor sizing problem.

\subsection{Chapter Summary}

This chapter has described several delay modeling hchniques and optimization algorithms that have been applied in the area of transistor sizing. The RC model seems to be the most common practice for modeling the delay of a circuit. Two different modeling techniques other than RC delay models have also been dis- 
cussed. The complexity and the efficiency of the optimization strategies associated with the delay models are included in the discussion. Another observation is that, in general, none of the available optimization routines will handle the transistor sizing problem directly. Modification or adjustment to the optimization routines or delay models is always needed. This kind of adjustment is in fact affecting the robustness of the original optimization strategy. 


\section{Chapter 3 \\ The Gate Level Transistor Sizing Problem}

Gate level transistor sizing uses the scaling factors associated with each gate as the design variables for the problem. In digital CMOS circuits, the lengths of the $\mathrm{n}$ and $\mathrm{p}$ channel transistors are usually set to their minimum dimension. Widths are directly proportional to the scale factor or the "gate size". Transistor sizes within the gate scale up or down in proportion to its scale factor. Solving the problem at the gate level helps in reducing the problem size. In this thesis, the transistor sizing problem is formulated as an area minimization problem under timing constraints. The set of constraints includes the requirement that the total propagation delay for any path withir the circuit has to satisfy a certain maximum time restriction, and that each gate size must lie between the minimum and maximum size of its type. The total propagation delay through a path is simply the sum of the individual gate delays along the path.

This transistor sizing problem will be discussed in two main areas: modeling and optimization strategy. Modeling involves the design and the construction of different area and delay models, while the optimization strategy involves the choice of an optimizer and the way that the models are linked to the optimizer.

\subsection{Modeling Delay and Area}

The delay and area models were crucial to the success and the efficiency of the optimization strategy. In this thesis, new area and delay models are used and are applied to the optimization routines. Then, they are tested for their accuracy and efficiency in transistor sizing problems. 
The Gate Level Transistor Sizing Problem 32

\subsubsection{Delay Models}

The delay models [15] used here are developed by other researchers and have not previously been used in any optimization as is done in this thesis. The delay models are nonlinear functions of the design parameters. To simplify delay estimation, approximately equal rise an.1 fall times for all types of gates are required. Rise time is defined to be the time for a waveform to rise from 10 percent to 90 percent of its steady-state value, while the fall time is the time for a waveform to fall from 90 percent to 10 percent of its initial value. Hence, for an inverter, the following expression is sequired:

$$
W_{p}=\alpha W_{n}
$$

where

$$
\begin{aligned}
& W_{p}=\text { width of the } p \text {-type device } \\
& W_{n}=\text { width of the } n \text {-type device } \\
& \alpha=\text { the size ratio of the n-type device to the p-type device required } \\
& \quad \text { for equal rise and fall tirses }
\end{aligned}
$$

In order to achieve equal rise and fall times, an accurate width ratio, $\alpha$, is indispensable. Hence, an accurate ratio of $\mu_{n}$ and $\mu_{p}$ must be known, where $\mu_{n}$ and $\mu_{p}$ are the carrier mobilities associated with the $n$ - and $p$-devices and are depended on the process parameters.

For NAND type and NOR type gates, the relationships between the widths of the n-type and p-type transistors are different from EQ 3.1. To obtain approximately equal rise ard fall times for these gates, the following equations must be used:

\section{NOR-types gates}

$$
W_{p}=\alpha W_{n} \theta
$$




\section{NAND-types gates}

$$
W_{p}=\frac{\alpha W_{n}}{\theta}
$$

where $\theta$ is the number of inputs for the gate. Under these assumptions, the delay, $d$, for any type of gate can be expressed as:

$$
d=f_{1}\left(\text { GateType;GateSize; } C_{L} ; \tau_{i n}\right)
$$

where $C_{L}$ is the capacitive load and $\tau_{\text {in }}$ is the input switching time for the gate. The output switching time, $\tau_{\text {out }}$, for the gate can also be expressed as:

$$
\left.\tau_{\text {out }}=f_{2} \text { (GateType;GateSize; } C_{L} ; \tau_{\text {in }}\right)
$$

Both $J_{1}$ and $f_{2}$ are functions in nonlinear form. For example, the delay through a 2 input Nand gate is expressed as:

$$
d_{\text {Nand } 2}=c_{0}+c_{1} \tau_{\text {in }}+\frac{c_{2} C_{L}^{2}}{s}+\frac{c_{3} C_{L} \sqrt{\tau_{i n}}}{s}+\frac{c_{4} C_{L}^{2} \sqrt{\tau_{i n}}}{s}
$$

where $c_{i}$ 's are the coefficients and $s$ is the size of the gate.

\subsubsection{Area Models}

In transistor sizing problems, most designers have focused heavily on the delay models and the mathematical formulation (or optimizer). They often assumed that the area model is a simple function of the sizes of the gates[16, 17]. The question is, can a gate increase its size without increasing the area that it takes? Standard cells have fixed cell height. If the gate width exceeds a certain limit, it cannot fit into a single slot of space any more. Splitting the transistors is required and uses up some extra area. However, sizing up a gate by a certain amount does not imply that the area of that gate goes up proportionally. It depends 
on the standard cell height, the ratio $\alpha$ discussed in the last section and in some cases, the number of inputs to the gate, $\theta$. Hence, the answer to the above question is that an increase in the size of a gate does not necessarily imply an increase in the area that it takes, which suggests iJcas for the development of new area models. Thus, new area model(s) are derived here to capture this gate area behaviour and to permit formulation of the transistor sizing problern for nonlinear optimization.

The new area models used in this thesis are based on a couple of parameters: the cell height, say $H$, the size ratio, $\alpha$, which is required to achieve approximately equal rise and fall times, and the number of inputs to the gate, $\theta$. Let's consider the layout of an inverter in Fig.3.1. The idea is similar for other types of gates. The inverter in Fig. 3.1a occupies a single slot of space in a standard cell at its current size. The cell height is fixed at its minimum size. Doubling the scale factor of this gate affects the widths of the p-type and n-type transistors. However. the increase does not affect the area that it takes according to the cell height and the ratio, $\alpha$. Since the new width still fits into a single slot of space as shown in Fig.3.1b, the increase in the gate size has no effect on the area. Doubling the size again (i.e. 4 times the original size) affects the total widths again according to $\alpha$. If the total widths of the transistors are now greater than the cell height, the gate has to be split because one single slot is obviously too small for the transistors. Thus, the area is doubled as shown in Fig.3.1c and 2 slots of space are required. Therefore, whether the gate needs splitting is determined by how much $W_{n}$ and $W_{p}$ are affected by the scale factor plus the ratio betweer them. 


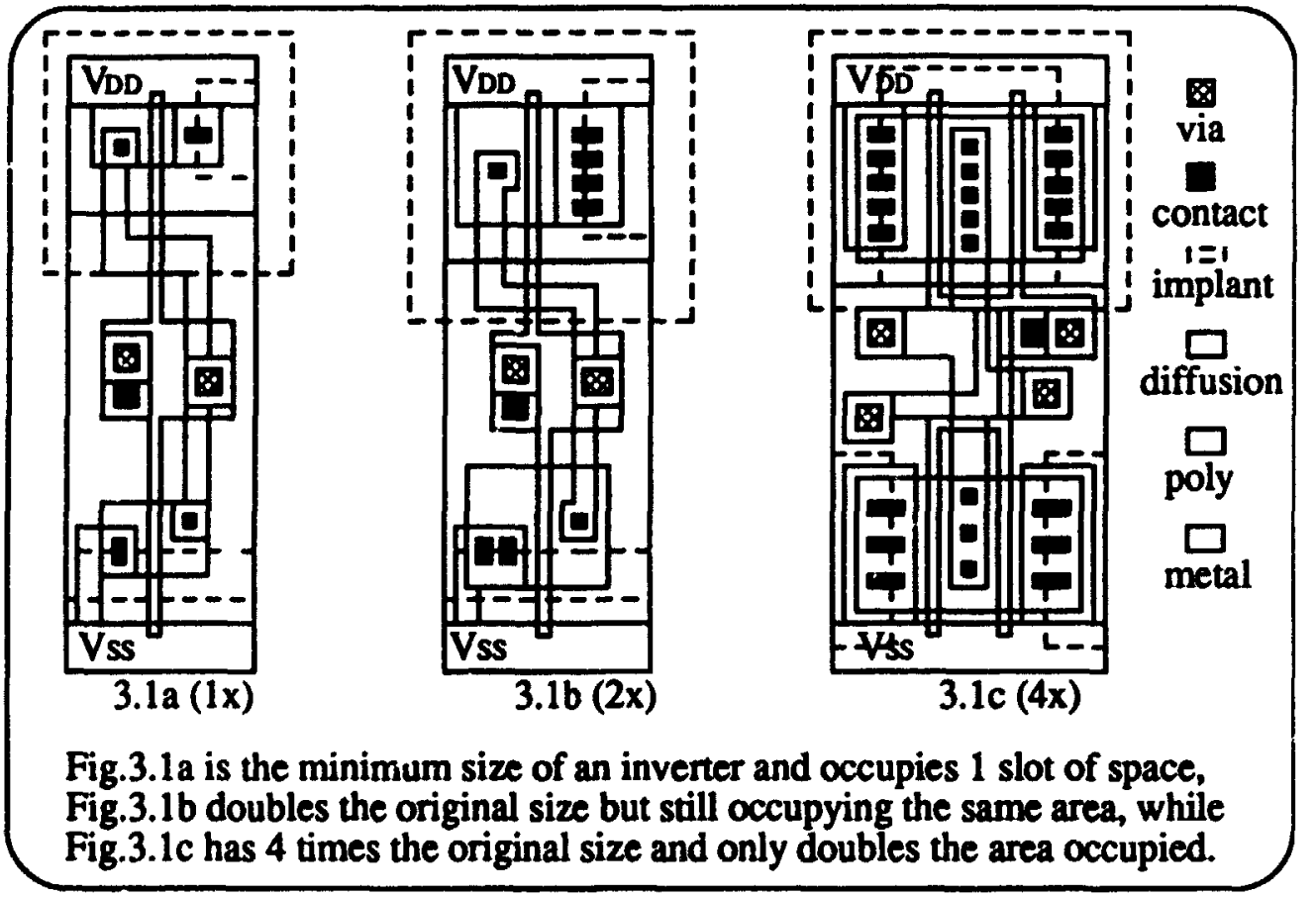

Eig. 3.1 Effect on area by increasing the size of an inverter in standard cell design.

Thus, the limit that decides a splitting of the gate totally depends on the height of the standard cell, $\mathrm{H}$ and the ratio $\alpha$. An increase in the size of a gate causes an increase in the occupied area only if the size exceeds some limit, say $S_{\text {critical. }}$ The amount of the area increase, say $\Delta A$, also depends on $H$ and $\alpha$. In this case, the relationship between the area and the size of a gate is best described as shown in Table 3.1: 
The Gate Level Transistor Sizing Problem 36

Table 3.1: The Staircase Area model

\begin{tabular}{|c|c|}
\hline Size of a gate & Area (in terms of slots of spaces) \\
\hline \hline$S_{\min } \leq S<S_{\text {critical }}$ & Min area \\
\hline$S_{\text {critical }} \leq S<2 S_{\text {critical }}$ & Min area $+\Delta A$ \\
\hline $2 S_{\text {critical }} \leq S<3 S_{\text {critical }}$ & Min area $+2 \Delta A$ \\
\hline$:$ & $:$ \\
\hline
\end{tabular}

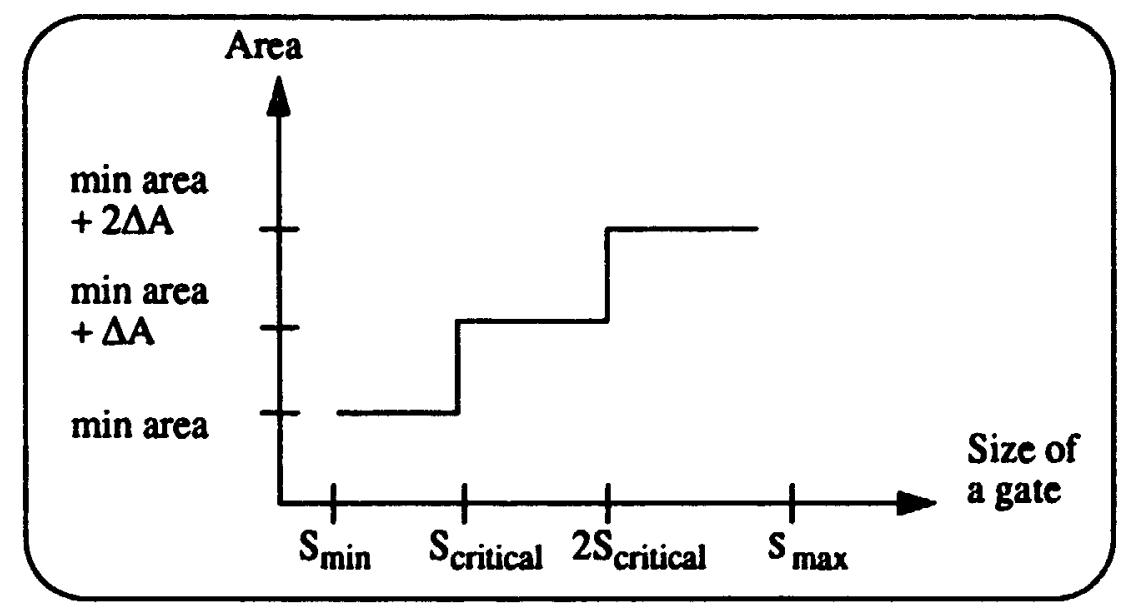

Fig. 3.2 The staircase area model.

Fig.3.2 shows why this new area model is called the staircase area model. $S_{\max }$ is the maximum size allowed for the gate. Notice that the staircase area model is nondifferentiable and special care has to be taken when applying an optimization strategy.

A similar idea is applied to the NAND type and the NOR type gates. They have the same set of parameters (i.e. $H$ and $\alpha$ ) plus an extra parameter $\theta$, which is thu number of inputs to the gate. Depending on these parameters, different values 
of $S_{\text {critical }} S_{\max }$ and $\Delta A$ are obtained for different types of gates. These values, together with those values for an inverter, are used in the objective function to determine the total area of the circuit.

A linear area model is used for comparison with the staircase model, as depicted in Fig. 3.3.

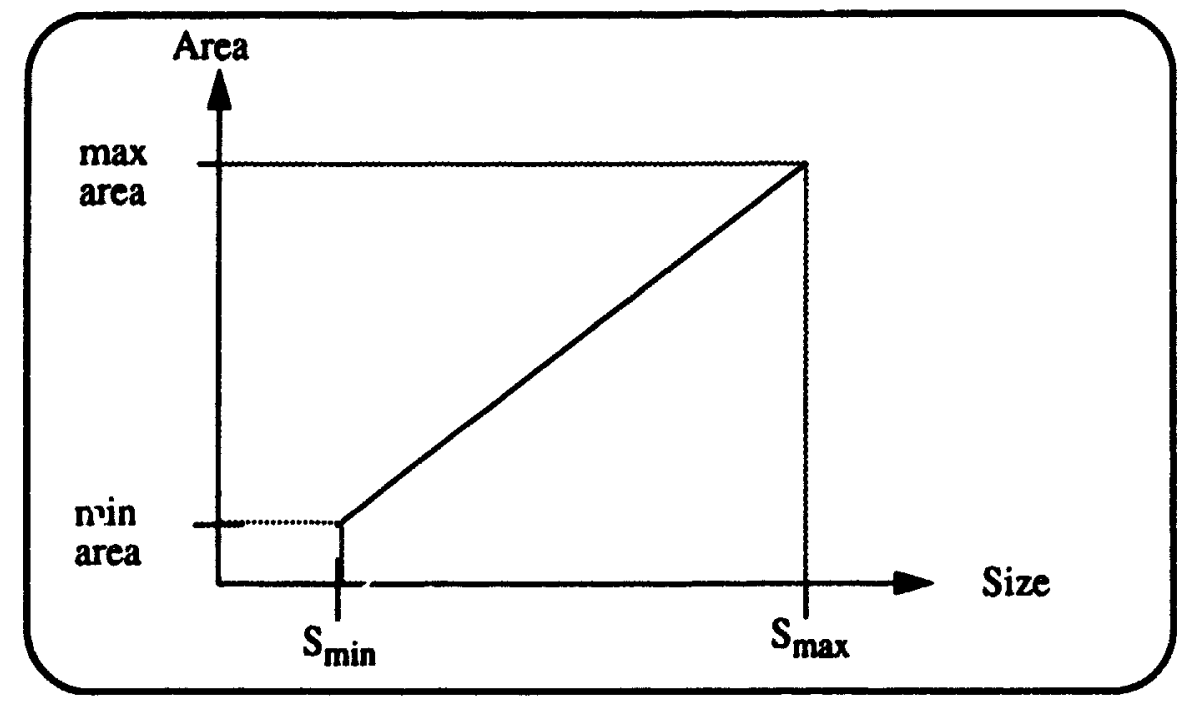

Fig.3.3 A linear area model.

Under the linear area model, an increase in the gate size has a direct proportional effect on the area that it occupied. The results using these two models are compared in Chapter 6.

\subsection{Variable and Parameter Declarations}

This is a gate level transistor sizing problem, so the variables are the scaling factors associated with the gates. Define:

$$
\begin{aligned}
& S_{i}=\text { scaling factor for gate } i \\
& A=\text { Area }=\sum_{i=1}^{n} f_{j}\left(S_{i}\right)
\end{aligned}
$$


where

$\mathbf{n}=$ total number of gates in the circuit

$f_{j}$ is an area function on gate $i$ and subscript $j$ depends on the type of gate

In order to formulate the objective function and the constraints of the optimization problem, extra variables and parameters are required. They are defined as follows:

$$
\begin{aligned}
d_{i} & =\text { delay through gate } i \\
& =D_{j}(S)
\end{aligned}
$$

where

$D_{j}$ is a delay function described in section 3.1.1. Subscript $j$ depends on the type of the gate.

$S$ is a vector of the variables $S_{i}$.

Define:

$\mathrm{TD}_{\mathrm{k}}=$ total propagation delay through path $\mathrm{k}$

$T_{\max }=$ user specified maximum allowable delay for the circuit

$S_{\min }=$ minimum size of a gate

$S_{\max }=$ maximum size of a gate

$S_{\text {critical }}=$ limit of the size of a gate determining whether a split of the transistors is required

Notice that the $S_{\min }, S_{\max }$ and $S_{\text {critical }}$ all have different values depending on the type of gate. 


\subsection{Problem Statement}

The problem is to minimize the total circuit area subject to a user-specified timing condition on the maximum propagation delay through the circuit. With the variables and parameters defined in the last section, the problem can be mathematically reformulated as:

minimize Area (see EQ 3.7)

s. t.

$$
\begin{gathered}
T D_{k} \leq T_{\max }, \forall k \\
S_{\min } \leq S_{i} \leq S_{\max }, \forall i
\end{gathered}
$$

Note that the objective function, the total area, can be composed of either staircase functions or linear functions.

\subsection{Choosing an Optimization Algorithm}

There are different solution techniques that can be applied to the transistor sizing problem as described in Chapter 2. Regardless of which algorithm is used, a good feasible initial solution improves the optimizer's performance substantially. An algorithm to generate a good initial point is developed in this thesis, and numerous optimizers could be chosen. However, the robustness of the optimizer is an important consideration. Among the available candidates, the Generalized Reduced Gradient (GRG) method as implemented in the LSGRG program [22], is chosen. The algorithm uses an elimination of variables technique to reduce the problem dimensionality, resulting in a speedier solution. Details of GRG method are given in Chapter 5. 


\section{Chapter 4}

\section{New Gate Level Transistor Sizing Algorithm}

The discussion in Chapter 3 leads to the conclusion that a new optimization strategy is needed. The new algorithm is generally described in Algorithm 4.1:

Step 1: $\quad$ Construct data structures for the node and gate information of the network.

Step 2: $\quad$ Construct delay equations for nodal evaluation.

Step 3: $\quad$ Provide an initial solution by estimating the critical path of the network, and the gate sizes along the estimated critical path.

Step 4: $\quad$ Solve the full nonlinear optimization problem with above information and set-up, starting at the initial point found in Step 3.

\section{Algorithm 4.1 General algorithm for solving the Gate Level TSP}

The new algorithm first creates a directed acyclic graph (DAG) format table to store the circuit structure. The nodes and gates of the circuit correspond to the nodes and edges of the network. Every edge in the network corresponds to a gate in the circuit and a node represents a primary input, a primary output or an interconnection point of the circuit. This forms an Activity On Arc (AOA) network. The delay calculation through each gate is set up according to the DAG table. The heuristic algorithm generates a feasible starting point by first estimating the critical path of the circuit, and then assigns initial sizes to the gates. In order to find an optimum or near optimum solution, the original problem is then reformulated into a nonlinear optimization problem. New variables are introduced and all the data and information are linked to the optimizer, in this case, LSGRG (Large Scale Generalized Reduced Gradient). Since a feasible initial point is provided by 
the heuristic algorithm, LSGRG solves the problem in Step 4 without leaving the feasible region. Therefore, an optimum or near optimum solution is found.

\subsection{Data Preparation}

For the purpose of demonstration, a small example called Examplel is solved. The input file is shown in Fig.4.1 and the circuit is shown in Fig. 4.2.

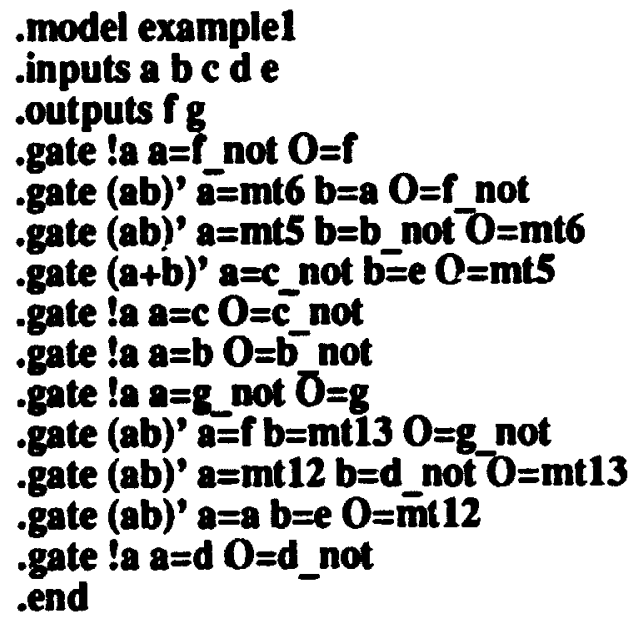

Eir.4.1 Circuit file for Examplel. 


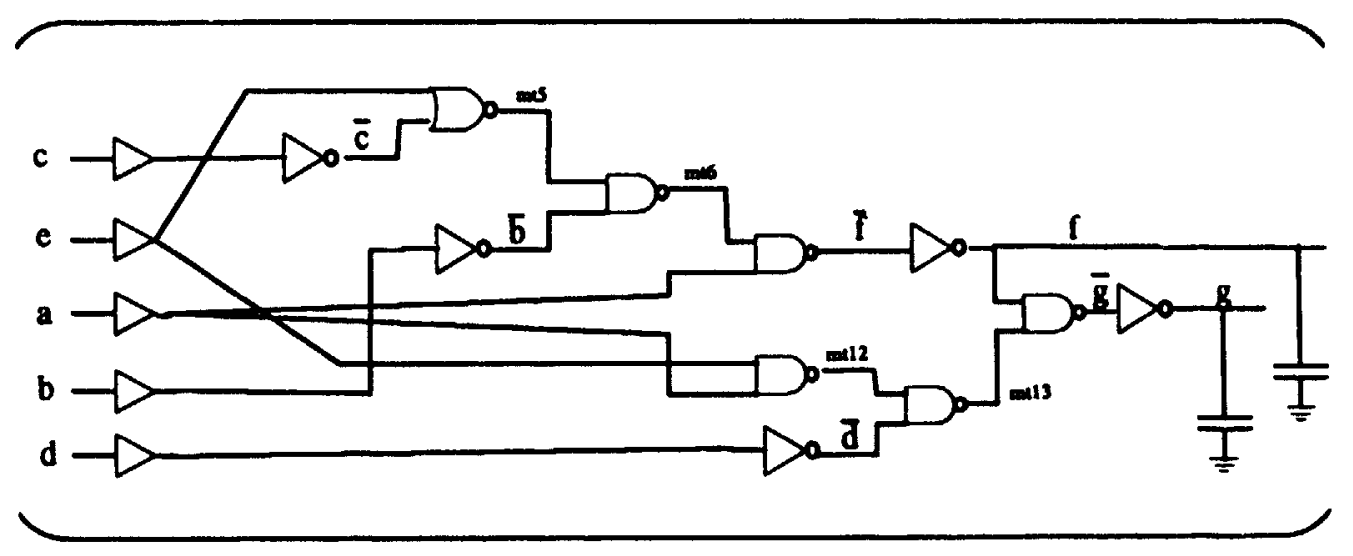

\section{Fig.4.2 Circuit diagram for Examplel.}

\subsubsection{Graph Construction}

The algorithm first reads in the circuit file and determines the number of inputs and outputs for the circuit. The first scan of the file determines the number of gates and nodes required and also determines the types of the gates in the circuit. A skeleton of the DAG format table is created. The directed acyclic graph corresponding to Example1 is shown in Fig. 4.3.

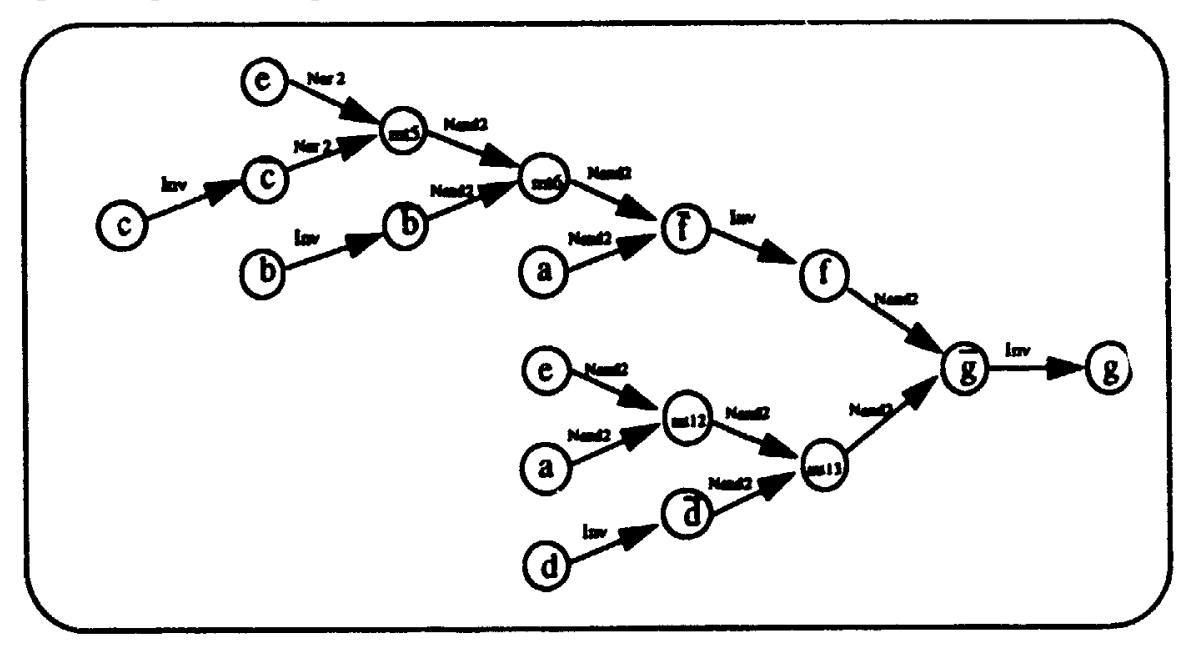

Eig.4.3DAG-Network model for Examplel. 
The second scan of the file assigns node and gate numbers and creates the DAG format table. The procedure to construct the table is similar to a Depth-First Search algorithm. Starting at the root (a primary output) and traversing down the branches, all necessary information is recorded. The procedure repeats the same steps for all primary outputs. A brief description of the procedure is given in Algorithm 4.2.

Step 1: Select a primary output gate and assign gate and node number. (Information is stored in the corresponding row of the table.) If no more primary output gates left, TERMINATE.

Step 2: $\quad$ Select an unvisited input from the output gate from step1. If all inputs have been visited, goto Step1.

Step 3: Identify the corresponding input gate.

Step 4: If the input gate has not been visited, assign gate and node number and store in table.

Step 5: $\quad$ Select an unvisited input from the gate in step3.

Recursively repeat step3 to step5 until the gate input belongs to the set of primary inputs. Goto Step2.

\section{Algorithm 4.2 Procedure to construct the DAG format table.}

The result (i.e. the table) for Example1 is shown in Fig.4.4. 


\begin{tabular}{|c|c|c|c|c|c|}
\hline TYPE & NODE & GATE & INPIIT & & OUTPUT \\
\hline $\begin{array}{l}\text { inv } \\
\text { nand2 } \\
\text { mnbuf } \\
\text { in } \\
\text { inbuf } \\
\text { nand2 } 2 \\
\text { jnv } \\
\text { inbuf } \\
\text { nor 2 } \\
\text { inbuf } \\
\text { inv } \\
\text { nand2 } \\
\text { inv } \\
\text { inbuf } \\
\text { nand2 } \\
\text { nands } \\
\text { inbuf } \\
\text { inbuf }\end{array}$ & $\begin{array}{l}1 \\
2 \\
10 \\
8 \\
9 \\
3 \\
6 \\
7 \\
4 \\
5 \\
11 \\
12 \\
17 \\
18 \\
13 \\
14 \\
15 \\
16\end{array}$ & $\begin{array}{l}1 \\
2 \\
0 \\
6 \\
0 \\
3 \\
5 \\
0 \\
4 \\
0 \\
7 \\
8 \\
11 \\
0 \\
9 \\
10 \\
0 \\
0\end{array}$ & $\begin{array}{l}\text { f_not } \\
\text { mit6 } \\
\text { in_a } \\
\text { b } \\
\text { in_b } \\
\text { mits } \\
\text { c } \\
\text { in_c } \\
\text { e } \\
\text { in_e } \\
\text { g_not } \\
\text { d } \\
\text { d } \\
\text { in_d } \\
\text { mit12 } \\
\text { e } \\
\text { in_e } \\
\text { in_a }\end{array}$ & $\begin{array}{l}\text { b_not0 } \\
\text { c_not0 } \\
\text { mt13 } \\
\text { d_not0 }\end{array}$ & 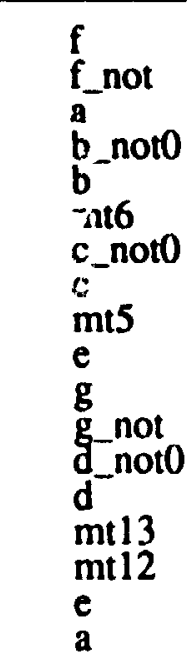 \\
\hline
\end{tabular}

Eig.4.4 Table of information for Exampled.

\subsubsection{Constructing Delay Equations}

Each gate has its own delay. The delay is calculated according to the delay models described in Chapter 3. Other information that should be taken into account includes the switching time of the input and the sizes of the fan-out gates, which affect the output capacitive load of the gate. To construct the delay equations, the DAG format table constructed in 4.1.1 is required. The prr -edure to construct the delay equations is described in Algorithm 4.3. 
Step1: Select a primary output and get its node number. TERMINATE if no more primary outputs left.

Step2: Select an unvisited input from the gate with its node number. If all inputs have been visited, goto Step4.

Step3: Trace the input node until primary input node is reached.

Step4: (Create delay equation)

- find out all the input nodes and fan-out gates for the current gate and its type

- create the delay equation according to the above information

If the current node element belongs to the primary output, goto Step1; otherwise goto Step5.

Step5: Go to its output node, goto Step2.

Algorithm 4.3 Procedure to construct the delay equations.

\subsection{Critical Path Analysis}

Finding the critical path of the circuit is an important issue in order to meet the timing specification and to achieve the smallest possible circuit in transistor sizing problems [4][5][19]. A path is defined to be critical if its total delay is the longest among all the paths in the network.

The major reason for estimating the critical path of the network is to obtain a feasible initial solution. A good feasible initial solution has the advantage that it provides a good starting point for the optimizer so the likelihood of terminating at an infeasible point is reduced. A good feasible initial solution which is reasonably close to an optimum solution reduces the number of iterations during the optimiza- 
tion process and increases the probability of terminating at the global optimum rather than a local minimum. A good feasible initial solution can be found by estimating the critical path of the circuit, and then calculating the total propagation delay through this path. Sizes along the critical path are set by a heuristic algorithm described in the next section, while other gates which are not on the critical path are set to their minimum sizes.

\subsubsection{Relative Delay Estimate method}

Finding the critical path of the circuit requires a knowledge of the delay through each gate. The delay is calculated according to the switching time of the input, the current gate size and the capacitive load of the gate. This information cannot be obtained without knowing the transistor sizes, but this contradicts the original purpose of finding a feasible initial solution for the problem since the sizes are not known. Thus, estimates for the sizes of the different types of gates are needed.

These estimates are obtained using a range of typical environments (i.e. switching input delays, sizes and capacitive loads). Under each environment, the delay through each type of gate is calculated. The total time for different types of gates within the same environment is also calculated. This value is used for calculating the relative delay estimates for each type of gate within the environment. The relative delay estimate is the delay through the gate over the total delay time for different types of gates as shown in brackets in Table 4.1. The relative delay estimates for each typical environment are averaged to arrive at the final estimate. Using the unitless relative delay estimates for the various devices, we can now estimate the location of the critical path.

The first three rows of Table 4.1 are the typical environments while the rest are the estimates for different devices. 
New Gale Level Transistor Sizing Algorithm 47

Table 4.1: Estimate the relative delay for each type of gate

\begin{tabular}{|c|c|c|c|c|c|c|c|}
\hline Tau_in & $0.5 n s$ & $0.25 \mathrm{~ns}$ & $0.3 \mathrm{~ns}$ & $0.25 n s$ & 0.4 ns & $0.35 n s$ & Relative \\
\hline Size & $2.5 \mu \mathrm{m}$ & $3.5 \mu \mathrm{m}$ & $1.8 \mu \mathrm{m}$ & $1.2 \mu \mathrm{m}$ & $4 \mu \mathrm{m}$ & $3 \mu \mathrm{m}$ & Delay \\
\hline$C_{\text {load }}$ & $0.0276 \mathrm{pF}$ & $0.0345 \mathrm{pF}$ & $0.015 \mathrm{pF}$ & $0.0138 \mathrm{pF}$ & $0.0483 \mathrm{pF}$ & $0.1035 \mathrm{pF}$ & Estimate \\
\hline Inverter & $\begin{array}{l}0.25867 n: \\
(7 \%)\end{array}$ & $\begin{array}{l}0.21241 \mathrm{~ns} \\
(6 \%)\end{array}$ & $\begin{array}{l}0.20356 \mathrm{~ms} \\
(5.7 \%)\end{array}$ & $\begin{array}{l}0.21838 \mathrm{~ns} \\
(5.6 \text { \%) }\end{array}$ & $\begin{array}{l}0.26136 \mathrm{~ns} \\
(7 \%)\end{array}$ & $\begin{array}{l}0.46236 \mathrm{~ns} \\
(7.8 \%)\end{array}$ & 0.65 \\
\hline Nand2 & $\begin{array}{l}0.38155 \mathrm{~ns} \\
(10.2 \%)\end{array}$ & $\begin{array}{l}0.33557 \mathrm{~ns} \\
(9.6 \%)\end{array}$ & $\begin{array}{l}0.32196 \mathrm{~ns} \\
(9.1 \%)\end{array}$ & $\begin{array}{l}0.33826 \mathrm{~ns} \\
(8.7 \%)\end{array}$ & $\begin{array}{l}0.38741 n s \\
(10.3 \%)\end{array}$ & $\begin{array}{l}0.70154 n s \\
(12 \%)\end{array}$ & 1.0 \\
\hline Nand 3 & $\begin{array}{l}0.53239 \mathrm{~ns} \\
(14.2 \%)\end{array}$ & $\begin{array}{l}0.47030 \mathrm{~ns} \\
(13.6 \%)\end{array}$ & $\begin{array}{l}0.53684 \mathrm{~ns} \\
(15.2 \%)\end{array}$ & $\begin{array}{l}0.65379 n s \\
(16.8 \%)\end{array}$ & $\begin{array}{l}0.50415 \mathrm{~ns} \\
(13.4 \%)\end{array}$ & $\begin{array}{l}0.90539 \mathrm{~ns} \\
(15.4 \%)\end{array}$ & 1.45 \\
\hline Nand4 & $\begin{array}{l}\text { 0.7342Ans } \\
(19.6 \%)\end{array}$ & $\begin{array}{l}0.65268 \mathrm{~ns} \\
(18.8 \%)\end{array}$ & $\begin{array}{l}0.75540 \mathrm{~ns} \\
(21.4 \%)\end{array}$ & $\begin{array}{l}0.92198 \mathrm{~ns} \\
(23.7 \%)\end{array}$ & $\begin{array}{l}0.69023 \mathrm{~ns} \\
(18.4 \%)\end{array}$ & $\begin{array}{l}1.21632 n s \\
(20.7 \%)\end{array}$ & 2.05 \\
\hline Nor2 & $\begin{array}{l}0.43476 \mathrm{~ns} \\
(11.6 \%)\end{array}$ & $\begin{array}{l}0.38516 n s \\
(11.1 \%)\end{array}$ & $\begin{array}{l}0.36953 \mathrm{~ns} \\
(10.5 \%)\end{array}$ & $\begin{array}{l}0.38620 \mathrm{~ns} \\
(10 \%)\end{array}$ & $\begin{array}{l}0.44235 \mathrm{~ns} \\
(11.7 \%)\end{array}$ & $\begin{array}{l}0.78749 \mathrm{~ns} \\
(13.49)\end{array}$ & 1.15 \\
\hline Nor3 & $\begin{array}{l}0.55839 \mathrm{~ns} \\
(14.9 \%)\end{array}$ & $\begin{array}{l}0.55191 \mathrm{~ns} \\
(15.9 \%)\end{array}$ & $\begin{array}{l}0.54196 n s \\
(15.4 \%)\end{array}$ & $\begin{array}{l}0.59041 \mathrm{~ns} \\
(15.2 \%)\end{array}$ & $\begin{array}{l}0.57108 \mathrm{~ns} \\
(15.2 \%)\end{array}$ & $\begin{array}{l}0.73768 \mathrm{~ns} \\
(12.5 \%)\end{array}$ & 1.5 \\
\hline Nor4 & $\begin{array}{l}0.84149 \mathrm{~ns} \\
(22.596)\end{array}$ & $\begin{array}{l}0.85767 n s \\
(24.7 \%)\end{array}$ & $\begin{array}{l}0.78986 \mathrm{~ns} \\
(22.4 \%)\end{array}$ & $\begin{array}{l}0.77479 \mathrm{~ns} \\
(20 \%)\end{array}$ & $\begin{array}{l}0.90097 \mathrm{~ns} \\
(24 \%)\end{array}$ & $\begin{array}{l}1.05103 \mathrm{~ns} \\
(18 \%)\end{array}$ & 2.2 \\
\hline Total & $3.74151 n s$ & $3.46572 \mathrm{~ns}$ & $3.51934 \mathrm{~ns}$ & $3.88384 \mathrm{~ns}$ & $3.75757 \mathrm{~ns}$ & S.86181ns & $10(100 \%)$ \\
\hline
\end{tabular}

\subsubsection{Critical Path Method}

The algorithm to estimate the critical path is the well-known Critical Path Method (CPM) [21]. An illustration of the CPM on Examplel is shown in Fig.4.5 and Table 4.2. 


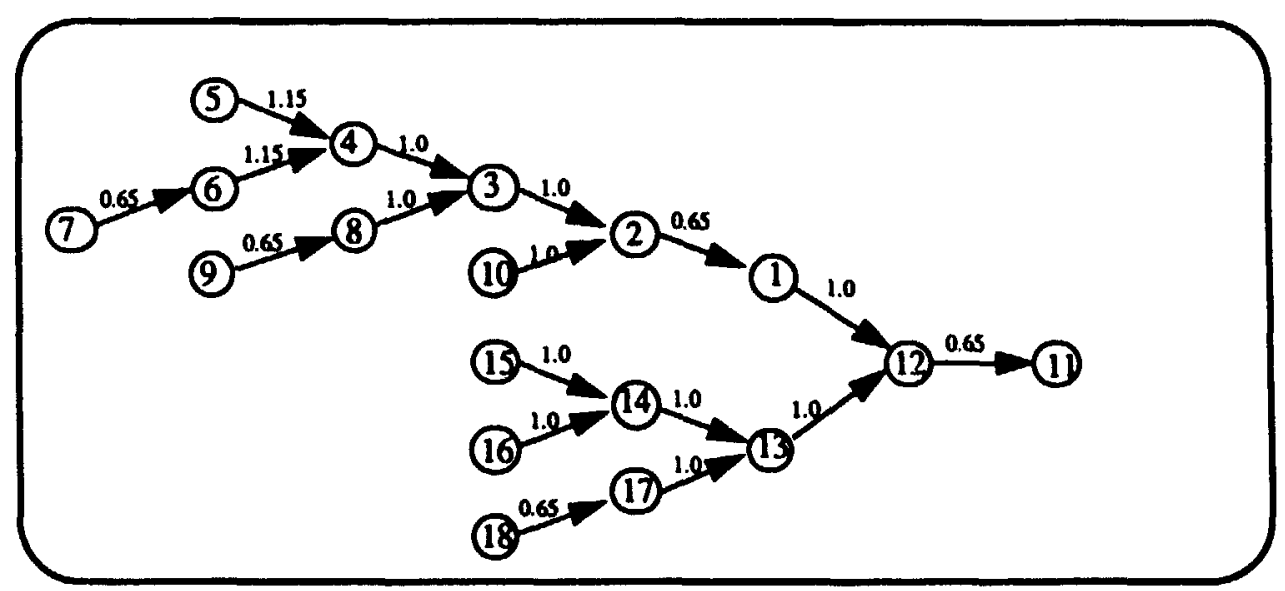

Eig.4.5 AOA network for Examplel showing the relative delay estimates.

Table 4.2: Calculation of event time for Examplel

\begin{tabular}{|l|c|c|}
\hline $\begin{array}{c}\text { Node } \\
\text { Number }\end{array}$ & $\begin{array}{c}\text { Earliest Event Time } \\
\text { (using relative delay } \\
\text { estimates) }\end{array}$ & $\begin{array}{c}\text { Latest Event Time } \\
\text { (using relative delay } \\
\text { estimates) }\end{array}$ \\
\hline 1 & 4.45 & 4.45 \\
\hline 2 & 3.8 & 3.8 \\
\hline 3 & 2.8 & 2.8 \\
\hline 4 & 1.8 & 1.8 \\
\hline 5 & 0 & 0.65 \\
\hline 6 & 0.65 & 0.65 \\
\hline 7 & 0 & 0 \\
\hline 8 & 0.65 & 1.8 \\
\hline 9 & 0 & 1.15 \\
\hline 10 & 0 & 2.8 \\
\hline
\end{tabular}


Table 4.2: Calculation of event time for Example1

\begin{tabular}{|c|c|c|}
\hline $\begin{array}{c}\text { Node } \\
\text { Number }\end{array}$ & $\begin{array}{c}\text { Earliest Event Time } \\
\text { (using relative delay } \\
\text { estimates) }\end{array}$ & $\begin{array}{c}\text { Latest Event Time } \\
\text { (using relative delay } \\
\text { estimates) }\end{array}$ \\
\hline 11 & 6.1 & 6.1 \\
\hline 12 & 5.45 & 5.45 \\
\hline 13 & 2.0 & 4.45 \\
\hline 14 & 1.0 & 3.45 \\
\hline 15 & 0 & 2.45 \\
\hline 16 & 0 & 2.45 \\
\hline 17 & 0.65 & 3.45 \\
\hline 18 & 0 & 2.8 \\
\hline
\end{tabular}

The result shows the critical path of the circuit. According to Fig.4.6, the critical path is: $(7 \rightarrow 6 \rightarrow 4 \rightarrow 3 \rightarrow 2 \rightarrow 1 \rightarrow 12 \rightarrow 11)$. Node 7 is the primary input node while node 1 and node 11 are both primary output nodes. 


\section{Slack Calculation}

$(7,6)=0.65-0-0.65=0$

$(6,4)=1.80-0.65-1.15=0$

$(5,4)=1.80-0-1.15=0.65$

$(9,8)=1.80-0-0.65=1.15$

$(8,3)=2.80-0.65-1=1.15$

$(4,3)=2.80-1.80 \cdot 1=0$

$(10,2)=3.80-0-1=2.8$

$(3,2)=3.80-2.80-1=0$

$(2,1)=4.45 \cdot 3.80 \cdot 0.65=0$

$(15,14)=3.45-0-1=2.45$

$(16,14)=3.45-0-1=2.45$

$(18,17)=3.45-0-0.65=2.8$

$(14,13)=4.45-1.0-1=2.45$

$(17,13)=4.45-0.65-1=2.8$

$(1,12)=5.45 \cdot 4.45 \cdot 1=0$

$(13,12)=5.45-2.0-1=1.45$

$(12,11)=6.1-5.45 \cdot 0.65=0$

Eig,4.6 Activity slack calculation for the network of Exampled.

\subsection{Gate Size Estimation}

Once the critical path of the network is estimated, each gate will be assigned a gate size (i.e. a scaling factor). This is a complicated task because the size of a gate affects the capacitive load of the previous gate(s), which in turn, affects the delay. Thus, gate sizes must be estimated very carefully.

Exponential growth of the gate sizes along the estimated critical path is assumed, based on the pattem of growth of the gate sizes in a chain of inverters [23]. To size a chain of inverters, the multiple buffer design is used. The stages under this design are assumed to be progressively larger by a constant factor of $f$. Let $Y$ be the ratio of the output capacitance to the input capacitance, then for minimum total delay, the constant factor $f$ is found to be: 


$$
f=e^{\frac{\ln Y}{n}}
$$

where $n$ is the number of inverters in the chain. That is, the scaling is exponentially increasing along the chain from input to output.

This characterization is applied in the general case for a chain of different types of gates. Although inaccurate for the general case, this characterization provides a reasonable initial estimate of the sizes. The algorithm is shown in Algorithm 4.4.

Step 1: $\quad$ Select the primary input node on the estimated critical path. Set Level $=1$.

Step 2: $\quad$ Trace the next output node on the estimated critical path. The gate between the two nodes is ascigned a size according to

$$
\text { size }=(\min \text { size }) * 1.2 \text { Level }-I
$$

If the output gate belongs to the same subcircuit, then

$$
\text { Level }=\text { Level }+1
$$

otherwise, Level remains the same.

Repeat Step2 until a primary output node is reached.

Step 3: Other gates not on the critical path are set to the minimum gate size.

\section{Algorithm 4.4 Algorithm to estimate the initial gate sizes,}

Now consider the function ( $m$ in size) $* 1.2^{\text {Level-1 }}$. The constant 1.2 is used because it results in a slow but steady rise in gate sizes along the estimated critical path. Together with the power index Level, it provides an exponential growth in 
gate sizes along the critical path. Moreover, increasing the gate sizes by large amounts does not imply a minimum total propagation delay for the network. Thus, in order to obtain a minimum size circuit and provide a close estimate to the optimum solution, a slow exponential growth function is desired. This idea is applied to Examplel and the results are shown in Fig.4.7.

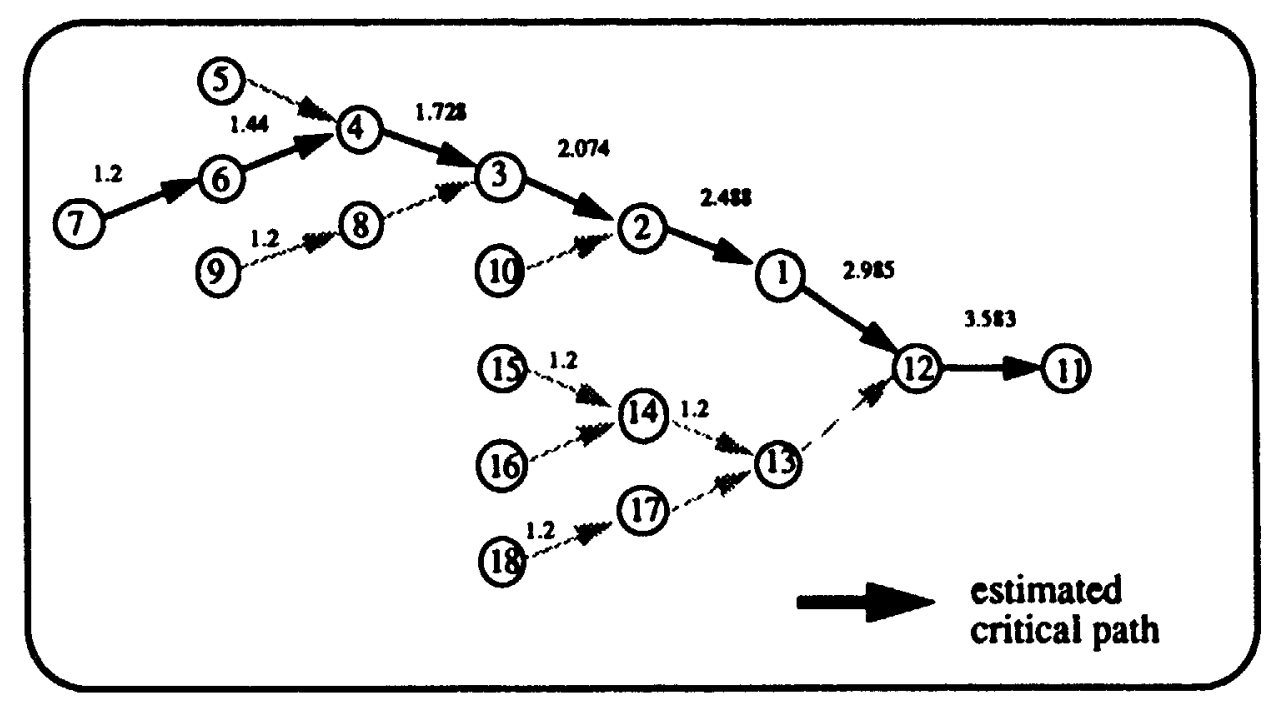

Eig.4.7 Estimates of the gate sizes are shown on edges for Examplel.

\subsection{Piecewise Linear and Cubic Approximation of the Area}

Recall from Section 4.1.2 that the new area model is a nondifferentiable staircase function of the gate sizes (scaling factors). Unfortunately, most optimization strategies assume continuous differentiability of the objective and the constraints functions. In order to use an efficient algorithm for the problem, modification of the staircase function is required. The method used in this thesis is a piecewise approximation.

To closely approximate the original objective function, a combination of 
linear functions and cubic polynomials is used. The idea is to use the linear parts of the staircase, and join them smoothly using cubic polynomials. There are four conditions required in order to have a continuously differentiable curve:

(i) the endpoints of the cubic polynomials and the neighbouring linear functions must coincide

(ii) the slopes at the endpoints of the cubic polynomials must equal the slopes of the neighbouring linear functions

This set of four equations implies that the required polynomial is a unique cubic polynomial.

There is another important point within the approximation process: the idea of the monotonically increasing function. Monotonically increasing means there is no flat or decreasing portion of the curve which could lead to a local minimum point. Thus, a careful setup of the linear functions is required so that the whole piecewise curve is monotonically increasing and is as close as possible to the original curve. For different types of gates, there are different staircase functions for the area as described in Chapter 3. The piecewise curve for any particular type of gate is obtained by adjusting a parameter $\alpha$, which is used to set up the coordinates for the end points of the linear pieces. These coordinates are used to generate the equations for all the linear and cubic pieces, and they also have an effect on the shape of the cubic pieces which are generated. A plot of the piecewise curve compared with the original staircase function is obtained to decide whether the new curve is acceptable or not. An example showing the piecewise curve for the inverter is shown in Fig.4.8. There is a small portion of the approximated curve which is underestimated. Although these areas cause inaccuracy, it is a necessary compromise in order to achieve a close estimate to the original curve while retaining the concept of a monotonically increasing function. 


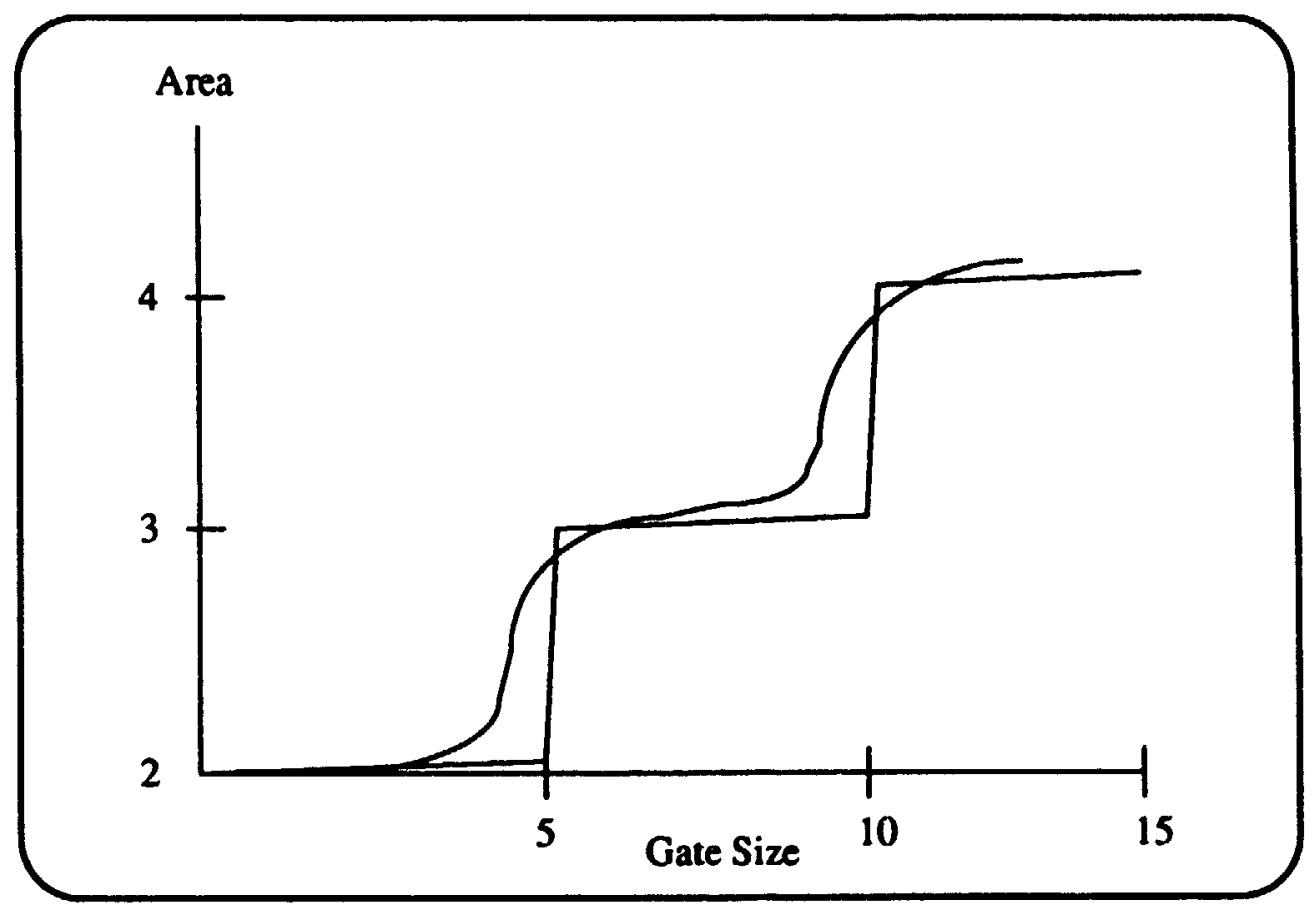

Eig.4.8 Piecewise Approximation of an Inverter.

The use of $\alpha$ to determine the linear pieces end points placement is graphically explained in Fig.4.9. Once the end points are set the linear and cubic piece equations are easily determined. 


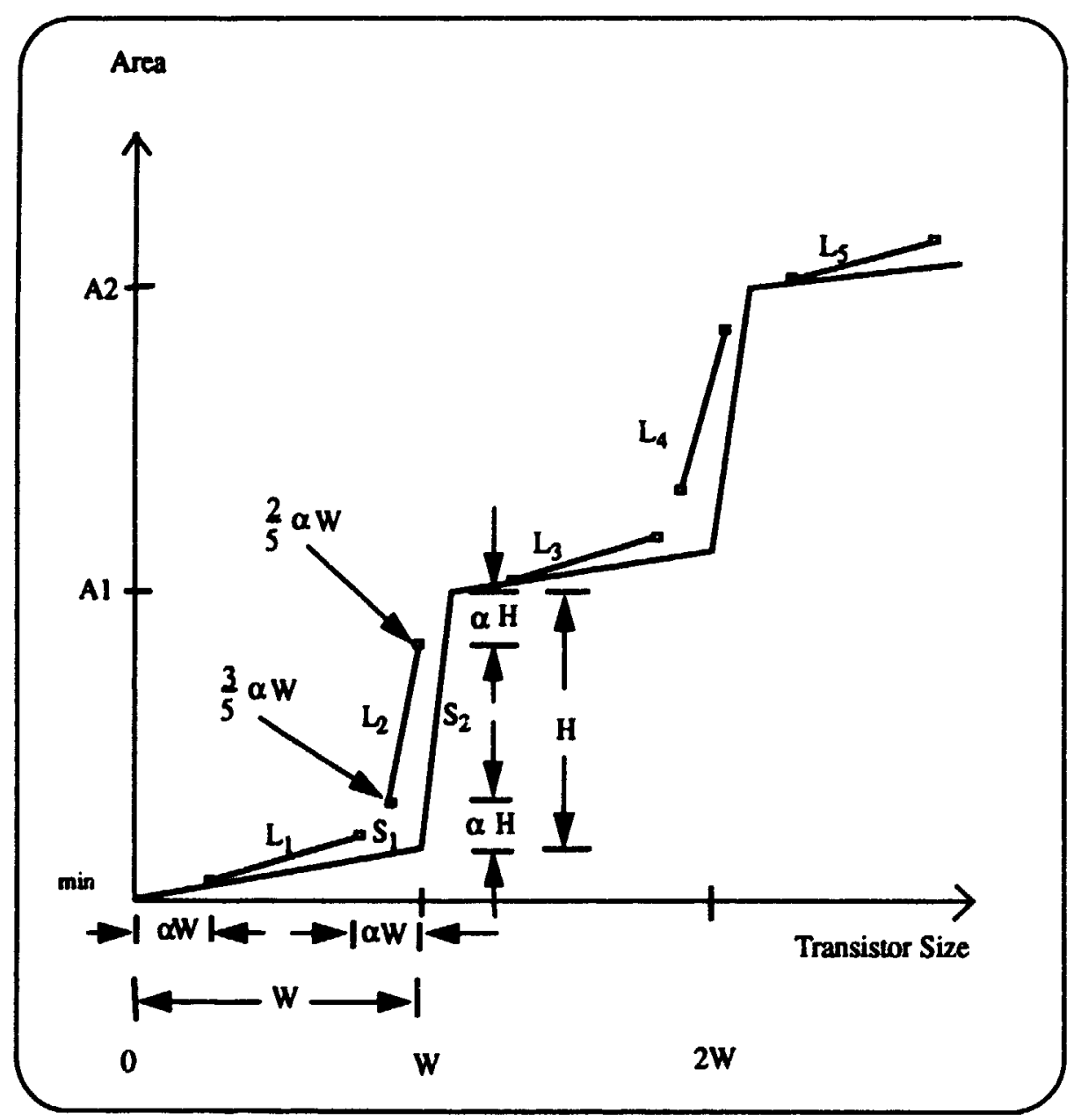

Eig.4.9 A diagram to explain the approximation of the staircase function.

Notice frum Fig.4.9 that $W$ and $A_{i}$ correspond to the $S_{\text {critical }}$ and $\Delta A$ respectively in Fig.3.2. The two end points for the vertical linear pieces are chosen as $\frac{2}{5}$ and $\frac{3}{5}$ of $\alpha \mathrm{W}$. These values are determined by testing and adjusting the vertical pieces so that a monotonically increasing function can be assured. 


\subsection{Chapter Summary}

In this chapter, a new gate level transistor sizing algorithm is presenteci. It solves the problem by first converting the circuit into a network model, and then by estimating the critical path of the network, it estimates an initial set of sizes applying to the optimizer. To handle the problem of nondifferentiable objective function, a combination of piecewise linear and curic functions is used. With the algorithms described in this chapter, all necessary information required by the optimizer is prepared. 


\section{Chapter 5 \\ Optimization}

\subsection{Formulation of the Nonlinear Programming Problem}

The final determination of the gate sizes is complex. Increasing the size of a gate to speed up one signal path may slow down another because of the capacitive loading effects. However, by reformulating the transistor sizing problem as a mathematical optimization problem, these global path interaci.uns can be taken into account.

In order to formulate the transistor sizing problem as a nonlinear optimization problem, a new set of variables associated with each node in the AOA network is required:

$$
t_{j}=\text { latest signal ar ival tir.te at node } j
$$

These new variables are useful in determining the set of violated constraints during the optimization process. The calculation of these variables is straight-forward from the network. For every node $j$ with incoming eiges $(i, j)$, the value of the new variables is determined by

$$
t_{j}=\max _{i}\left\{t_{i}+d_{i j}\right\}
$$

where for every node $i$ which is connected to node $j, d_{i j}$ is the delay through the edge $(i, j)$ and is calculated using the delay equations set up in Chapter 4.

\subsection{Timing Constraints}

The timing constraints in the optimization problem are set up using Eq.5.1 and by reference to the graphical network. Every pair of nodes connected by an edge corresponds to a constraint in the nonlinear optimization problem. A small example is shown in Fig.5.1. 


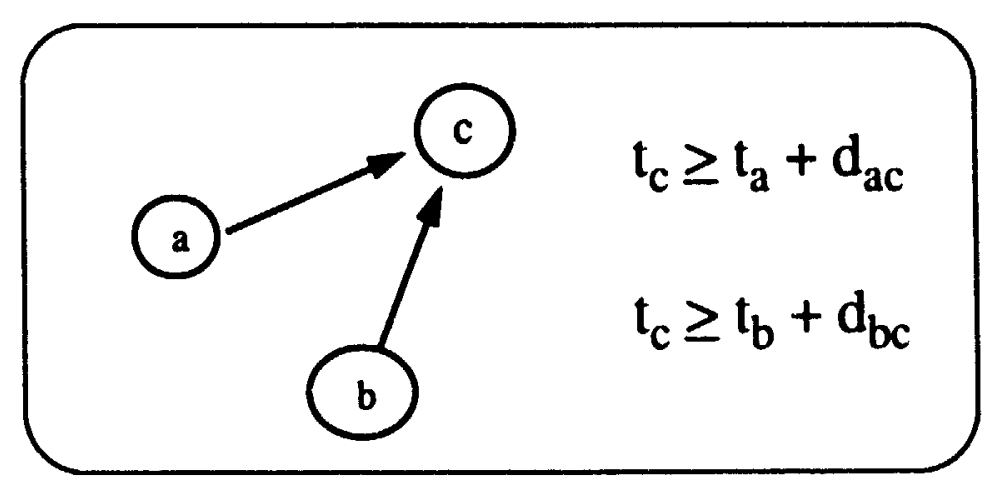

Eig.5.1 Illustration of setting up network constraints.

There are special cases to consider when constructing the set of timing constraints. When the node element is a primary input, the latest arrival time for the node is the delay through the smallest size buffer with capacitive load depending on its fan-out gates. For example, if the node element "a" in Fig.5.1 is a primary input, then this constraint is written as:

$$
t(a)=d_{\text {buffer }}(a)
$$

because the latest arrival time for this node is the time through a minimum size buffer. Whenever a node element belongs to the set of primary outputs, the overall constraint on the total delay takes the form:

$$
t_{\max } \geq t_{\text {primary_ouput_node }}
$$

The various delay equations complete the set of constraints, and are set up as described in Chapter 4. The timing constraints for Fxamplel are shown in Fig.5.2. 
Optimization 59

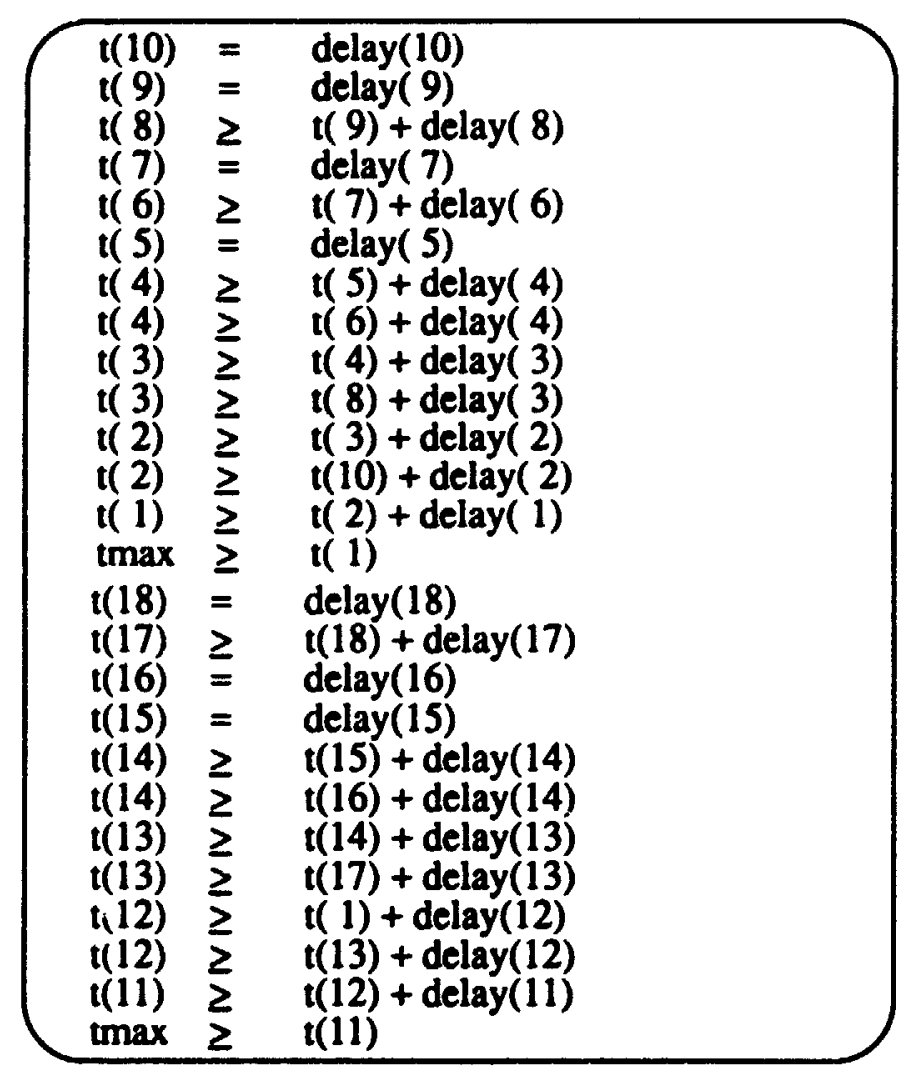

Eig. 5.2 Set of constraints for Examplel.

\subsection{Optimizer}

The last step is to submit the optimization problem and an initial point to an optimizer in order to solve the problem. The sptimizer used in this thesis is called LSGRG [22], which uses an optimization method called the Generalized Reduced Gradient (GRG) method. 


\subsubsection{Generalized Reduced Gradient method}

Generalized Reduced Gradient algorithms were introduced by Abalie and Carpentier [20]. GRG uses a 2-phase approach. Phase 1 of the method tries to tind a feasible initial point, and phase 2 tries to find the optimum without leaving the feasible region. However, if a feasible initial point is provided by the user, GRG will go directly to phase 2 . Since the method uses the active constraints of a problem to express basic variables in terms of the nonbasic variables ${ }^{1}$, GRG algorithms solve the original problem by solving a sequence of sub-problens, where the subproblems are obtained by direct elimination of variables. The reduced objective then becomes a function of the nonbasic variables alone and its gradient is called the reduced gradient. The reduced gradient is used to determine a search direction for the nonbasic variables, and a line search is used to determine the size of the step taken in the search direction. During the line search, a basic variable may equal or violate one of its bounds. If this happens, a new partitioning of the variables is defined. The process is repeated on the new reduced objective.

Let the nonlinear optimization problem be

$$
\begin{aligned}
& \min f(x) \\
& \text { s.t. } \\
& c_{i}(x)=0, i=1, \ldots, m . \\
& l_{j} \leq x_{j} \leq u_{j}, j=1, \ldots, n .
\end{aligned}
$$

where $\mathrm{x}$ is a vector of variables, $f$ is the objective function and the $c_{i}$ 's are the constraints. $l_{j}$ and $u_{j}$ are lower and upper bounds for variable $x_{j}$. All inequality constraints have been rephrased as equality constraints by using slack or surplus variables, which are already included in $x$. Variables are divided into basic, $x_{b}$ and nonbasic, $x_{n b}$ variables. The constraints can be written as $c_{i}\left(x_{b}, x_{n b}\right)=0$. The Jacobian matrix of $\mathbf{c}$ is also partitioned into

1. When solving a system of equations: $A x=b$, the variables that are forced onto a bound are called the nonbasic variables, the others are called the basic variables and they form a basis. 


$$
\frac{\partial c}{\partial x}=\left(\frac{\partial c}{\partial x_{b}}, \frac{\partial c}{\partial x_{n b}}\right)=\left(B, B_{n b}\right)
$$

Let $\mathbf{X}^{\prime}$ be th: current values of the variables. Then the specific variables to be chosen as the basic variables must be selected so that $B$, evaluated at $X^{\prime}$, is nonsingular. In this case, the constraints can be solved (at least conceptually) for $x_{b}$ in terms of $x_{n b}$ to yield the basic variables as a function of the nonbasic variables. The objective function is then reduced to a function of nonbasic variables alone, $F\left(x_{n b}\right)$. The new objective function $F$ is called the reduced objective and its gradient $\nabla F$ is called the reduced gradient.

GRG algorithms solve the original problem by solving a sequence of reduced problems. These reduced problems are usually solved by methods which use the reduced gradient $\nabla F$. At each iteration, the reduced gradient is computed as follows:

(i) solve $B^{T} \lambda=\frac{\partial f}{\partial x_{b}}$ for the multiplier vector $\lambda \in R^{m}$.

(ii) compute the reduced gradient by $\frac{\partial F}{\partial x_{a b}}=\frac{\partial f}{\partial x_{m b}}-B_{a b}^{T} \lambda$

A subset of the nonbasic variables, which are called the superbasic variables, $x_{\mathbf{s}}$, are varied in the current iteration. The reduced gradient with respect to the nonbasic variables, $\frac{\partial F}{\partial x_{n b}}$, is used only to determine if one of the components of $x_{n b}$ should be released from a bound to join the superbasic set. This decision can be made at each iteration or after an optimization over the current superbasic variables is completed within a loose tolerance. In either case, the reduced gradient with respect to the current superbasic variables, $\frac{\partial F}{\partial x_{a}}$, is used to form a search direction, $\bar{d}$. A one dimensional search is used to find the values of $\alpha$ which solves:

$$
\min _{\alpha \geq 0} F\left(x_{n b}+\alpha \bar{d}\right)
$$

Notice that the reduced objective function $F\left(x_{n b}\right)=f\left(x_{b}, x_{n b}\right)$ is a function of the nonbasic variables alone, which implies that the function $F\left(x_{n b}+\alpha_{i} \bar{d}\right)=f\left(x_{b}, x_{m b}+\alpha_{i} \bar{d}\right)$ and therefore, $x_{b}$ is determined by not only the 
nonbasic variables, $x_{n b}$ but also the search direction, $d$. These variables satisfy the system of equations

$$
c(x)=c\left(x_{b}, x_{n b}\right)=c\left(x_{b}, x_{n b}+\alpha_{i} \bar{d}\right)=0
$$

where $x_{n b}, \alpha$ and $d$ are chosen and $x_{b}$ is solved for. If $x_{b}$ appears nonlinearly in any constraint, then this system of equations must be solved by an iterative procedure, e.g. a variant of the Newton's method.

If the initial point $x_{0}$ is infeasible, GRG uses a 2-phase approach. Phase 1 attempts to determine a feasible point or show that there is none. Phase 2 of the algorithm optimizes the original objective if a feasible point is found.

\subsubsection{Advantages of the GRG method}

The GRG algorithm can be applied directly to the original problem without any variables or problem transformation. It is very robust. Furthermore, by expressing basic variables in terms of the nonbasic variables, the number of variables in the problem is reduced. Hence, the problem size is reduced and the algorithm can solve the problem more quickly. 


\section{Chapter 6 \\ Implementation and Experimental Results}

This chapter outlines the implementation of the algorithms discussed in chapter 4. The software prototype is then used for the purposes of experimentation and comparison with a fixed cell library approach.

\subsection{The Software Prototype}

The overall structure of the software prototype is shown in Fig. 6.1. It is composed of the following 5 modules: Constructor, Parsers, Estimator, Solver and Evaluator.

Constructor: This is the first module of the software prototype. The circuit file is read and is converted into different data structures. The DAG format table, which will be frequently used later, is created at this time.

Parsers: Together with the delay and area models, the DAG format table is used here to construct the objective function and the set of constraints required by the optimizer. The delay equations, part of the set of constraints, are also created.

Estimator: The critical path is estimated and the initial gate sizes are established.

Solver: The optimizer used in this prototype is called LSGRG. 
Evaluator: The optimizer output (in terms of gate sizes) is evaluated to determine the actual total area of the circuit. The total area from the Fixed Cell Library approach is also calculated. Finally, a summary of all the nodal information (i.e. the latest node arrival time and the nodes capacitances) and the gate information (i.e. initial and final gate sizes, gate type, actual area, input switching time, delay and capacitances of the gates) is printed.

A post processing step is executed here to make any necessary adjustment to the final solution. Details are to be shown in later section. 


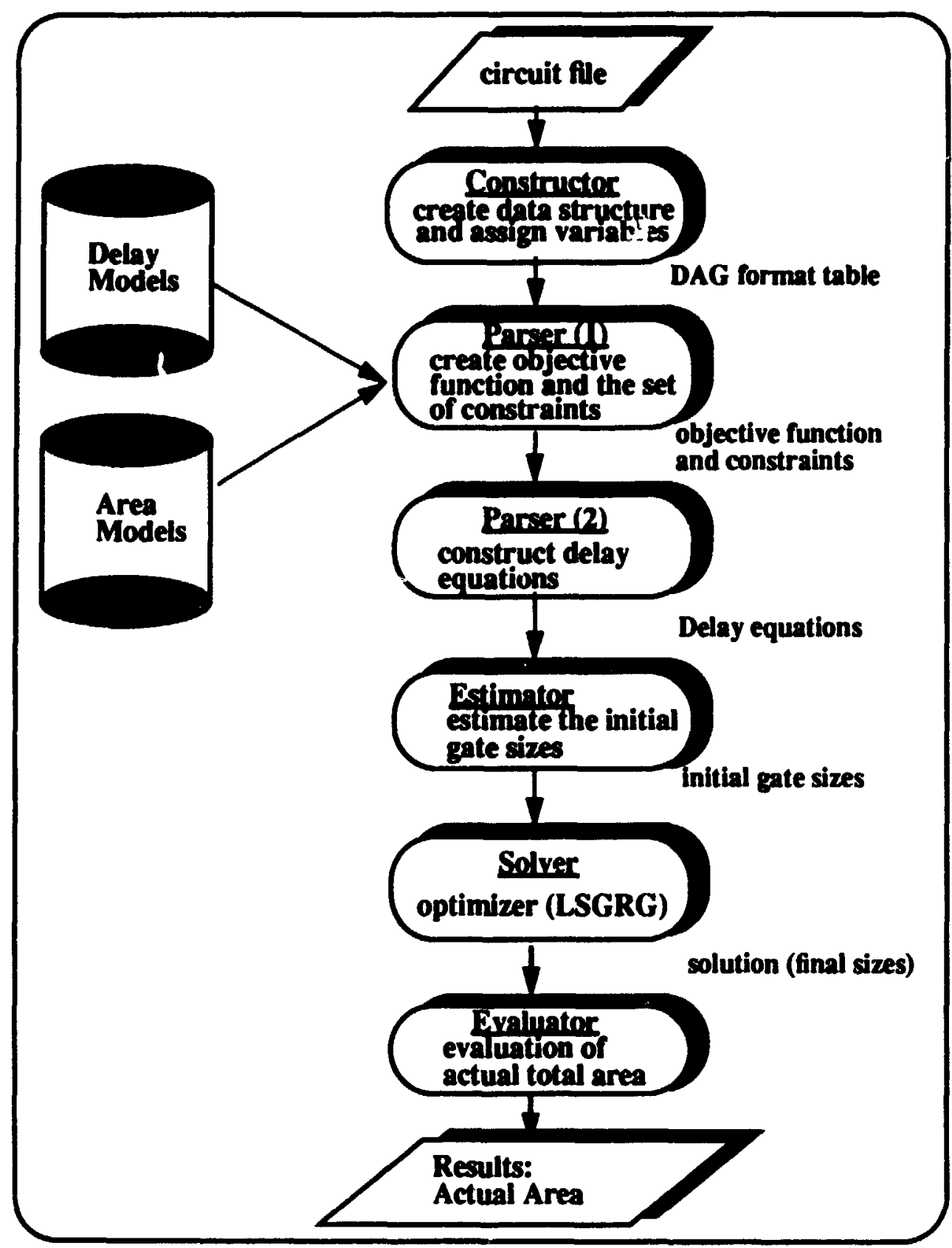

Eig.6.1 Elow Chart for the Transistor Sizing software prototype. 
This software prototype is implemented in FORTRAN, the same language used in the optimizer, LSGRG. The prototype runs under the UNIX operating system on a SUN SPARCstation 2. Some of the important subroutines can be found in the Appendix D. A brief user guide is also provided in the Appendix B.

\subsection{Experimental Procedure}

\subsubsection{Experimental Setup}

Four test problems, covering a range of problem sizes, are used in these experiments. The circuit files for the examples can be found in the Appendix $\mathrm{C}$. Examplel is a small example having 11 gates, 5 primary inputs and 2 primary outputs. It is mainly used as a working example throughout this thesis, and it has a tree-like network structure. Example2 is also a small size example which has 9 gates, 4 primary inputs and 2 primary outputs. This example tests the validity of the procedure on a different network structure. It has a non-tree structure but maintains the acyclic property. Example 3 and Example 4 are medium and larger size test problems. Example 3 contains 28 gates, 5 primary inputs and 3 primary outputs while Example 4 contains 136 gates, 36 primary inputs and 16 primary outputs. Both of these examples have a non-tree structure. For each test problem, the results obtained using the staircase area model and the linear area model are compared. $A$ comparison of the total area between the actual area and the Fixed Cell Library approach is also shown. These comparisons are used to demonstrate the effectiveness of the new staircase area model used in this thesis.

In order to determine the actual area for different types of gates, values of $S_{\text {critical }}, S_{\min }, S_{\max }$ and $\Delta A$ are required. Recall from Chapter 3 that jifferent types of gates have their own sets of values depending on the standard cell height, $H$, the 
width ratio between the $n$ - and $p$-type devices, $\alpha$ and the number of inputs to the gate, $\boldsymbol{\theta}$. For experimental purpose, these values are set as shown in Table 6.1 below:

Table 6.1: Values used for calculating the actual area of gates

\begin{tabular}{|c||c|c|c|c|c|}
\hline Type of Gate & $\mathrm{S}_{\min }$ & $\mathrm{S}_{\max }$ & $\mathrm{S}_{\text {critical }}$ & $\min$ Area & $\Delta \mathrm{A}$ \\
\hline \hline Inverter & 1.2 & 15.0 & 5.0 & 2.0 & 1.0 \\
\hline NAND2 & 1.2 & 9.0 & 3.0 & 3.0 & 2.0 \\
\hline NAND3 & 1.2 & 9.0 & 3.0 & 4.0 & 2.0 \\
\hline NAND4 & 1.2 & 9.0 & 3.0 & 4.0 & 2.0 \\
\hline NOR2 & 1.2 & 9.0 & 3.0 & 3.0 & 2.0 \\
\hline NOR3 & 1.2 & 9.0 & 3.0 & 4.0 & 2.0 \\
\hline NOR4 & 1.2 & 9.0 & 3.0 & 4.0 & 2.0 \\
\hline
\end{tabular}

\subsubsection{Optimization process}

Before the optimizer reaches a final solution, it has gone through a number of iterations. Each iteration involves processes like estimating the reduced gradient, searching for the steepest descent direction and calculating the step size along the search direction, etc. The following shows the iterations for Examplel using LSGRG.

ITN OBJECTIVE BDD SUPER DNF NORM RED HESSTAN BASIS UP STEP DEC NO. FUNCTION CONS BASIC CONS GRADIENT COND NO COND NO DATE SIZE STEP
$03.893426 \mathrm{E}+011960$
8.35E-01 1.00E+00 1.90E+01 F $0.00 E+00$
$13.529979 \mathrm{E}+0119,0$
$1.42 E+001.00 E+001.90 E+01$ F $8.20 E+00$
$23.395451 E+01194$
$1.51 \mathrm{E}+001.00 \mathrm{E}+001.90 \mathrm{E}+01$ F $4.00 \mathrm{E}-01$
$33.317123 E+011940$
$1.52 E+001.00 E+001.90 E+01$ F $2.11 E-01$ 


\begin{tabular}{|c|c|c|c|c|c|c|c|}
\hline 4 & $3.254784 E+0120$ & 3 & $\mathbf{0}$ & $5.31 E+00 \quad 1.18 E+01$ & $1.33 \mathrm{E}+03$ & \multicolumn{2}{|c|}{ QN 5.10602} \\
\hline \multicolumn{8}{|c|}{ NEWTON FAILURES - PIVOT PERCENTAGE HAS BEEN INCREASED TO 5.000D-01 } \\
\hline 5 & $3.254696 \mathrm{E}+0120$ & 3 & 0 & $5.35 E+00 \quad 1.00 E+00$ & $1.27 E+03$ & $\mathbf{F}$ & 1.00600 \\
\hline 6 & $3.253591 E+0120$ & 3 & 0 & $5.90 \mathrm{E}+00 \quad 1.00 \mathrm{E}+\infty 0$ & $1.46 \mathrm{E}+03$ & $\mathbf{F}$ & $6.621 ;-08$ \\
\hline 7 & $3.252883 \mathrm{E}+0120$ & 3 & $\mathbf{0}$ & $6.38 E+00 \quad 1.00 E+\infty 0$ & $1.63 E+03$ & $\mathbf{F}$ & $0.625-08$ \\
\hline 8 & $3.252791 E+0120$ & 3 & 0 & $6.45 \mathrm{E}+00 \quad 1.00 \mathrm{E}+00$ & $1.66 \mathrm{E}+03$ & $\mathbf{F}$ & $3.57 \mathrm{E}-09$ \\
\hline 9 & $3.251938 E+0120$ & 3 & $\mathbf{0}$ & $7.246+00 \quad 1.00 E+00$ & $1.93 \mathrm{E}+03$ & $\mathbf{F}$ & $5.27 k-08$ \\
\hline & $3.251707 \mathrm{E}+0120$ & 3 & $\mathbf{0}$ & $7.51 E+001.00 E+00$ & $203 E+103$ & $\mathbf{F}$ & $6.58 k-09$ \\
\hline 11 & $3.251491 \mathrm{E}+0120$ & 3 & 0 & 7.77E+00 1.00E+00 & $2.12 E+03$ & $F$ & 1.10200 \\
\hline \multicolumn{8}{|c|}{ NEWTON FALURES -. PIVOT PERCENTAGE HAS BEEN DNCREASED TO 8.0000 D-01 } \\
\hline & $3.251389 E+0120$ & 3 & 0 & $7.93 \mathrm{E}+001.00 \mathrm{E}+00$ & $2.17 \mathrm{E}+03$ & $r$ & 2.64E-09 \\
\hline & $3.251333 \mathrm{E}+0120$ & 3 & 0 & $8.01 E+001.00 E+n 0$ & $220 \mathrm{E}+03$ & $\boldsymbol{F}$ & 2.58E-09 \\
\hline \multicolumn{8}{|c|}{ FRACTIONAL CHANGE DN OBJECTIVE LESS THAN 1.00000E-04 } \\
\hline \multicolumn{8}{|c|}{ FOR 2 CONSECUTIVE ITERATIONS -- FORCLNG CONSBS INTO SEAKCH MODE: } \\
\hline & $3.251297 \mathrm{E}+0120$ & 4 & 0 & 7.13E-01 1.00E $+\infty$ & $1.20 \mathrm{E}+01$ & $\mathbf{F}$ & $4.29 \mathrm{E}-04$ \\
\hline
\end{tabular}

Starting from the first iteration (i.e. ITN NO. 0), notice that the number of infeasible constraints (INF CONS) is zero. This number indicates that a feasible initial solution is provided to the optimizer. Furthermore, a column of zeros under the INF CONS shows that the GRG method solves the problem without leaving the feasible region. Another point to notice is the pivot percentage change during the solution. The pivot sizes affect the performance of the algorithm. This pivot value is originally set to a small value (close to zero). This allows greater flexibility in choosing variables from bounds, which can optimize the improvement in the objective function value. The only drawback is the high basis condition number (BASIS COND NO), which indicates a slower convergence rate on Newton iterations, hence an increase in the chance of Newton failure, causing the algorithm to take smaller steps (STEP SIZE) in later iterations.

There are two acceptable choices to claim a successful termination. The first one is by satisfying the Kuhn-Tucker conditions within a very small tolerance, 
and the second one is to get a very small total fractional change in the objective for three consecutive iterations. The former guarantees the solution is a global optimum if the objective function and the set of constraints are convex functions over a convex set, otherwise a local minimum point is found instead. It is the best termination outcome. The latter one finds a solution inside the feasible region but the solution is most likely a local minimum point.

The stopping condition for this test problem belongs to the latter case, hence, global optimality of this solution cannot be assured. However, by performing more tests using different starting points, the probability that the solution is close to the optimum may be improved if the results fall within a small region. The experiments showed that if the starting points are close to the minimum setting of the gate sizes and are linearly or exponentially increasing from a primary input to a primary output, the solutions are located within $5 \%$ in the neighbourhood of the final solution shown above. On the other hand, starting points far from the minimum region yielded local minimum points which have higher objective function values. However, the global optimality of the solution cannot be assured for this problem.

Although the example shown above did not achieve guaranteed local optimality for the solution, other examples terminated at points satisfying the KuhnTucker conditions. In those cases, a locally optimal solution is found. Results for the test problems are listed in Appendix C.

\subsubsection{Optimization Results}

Once again the network with the gate and node information for Example 1 is shown in Fig.6.2. 


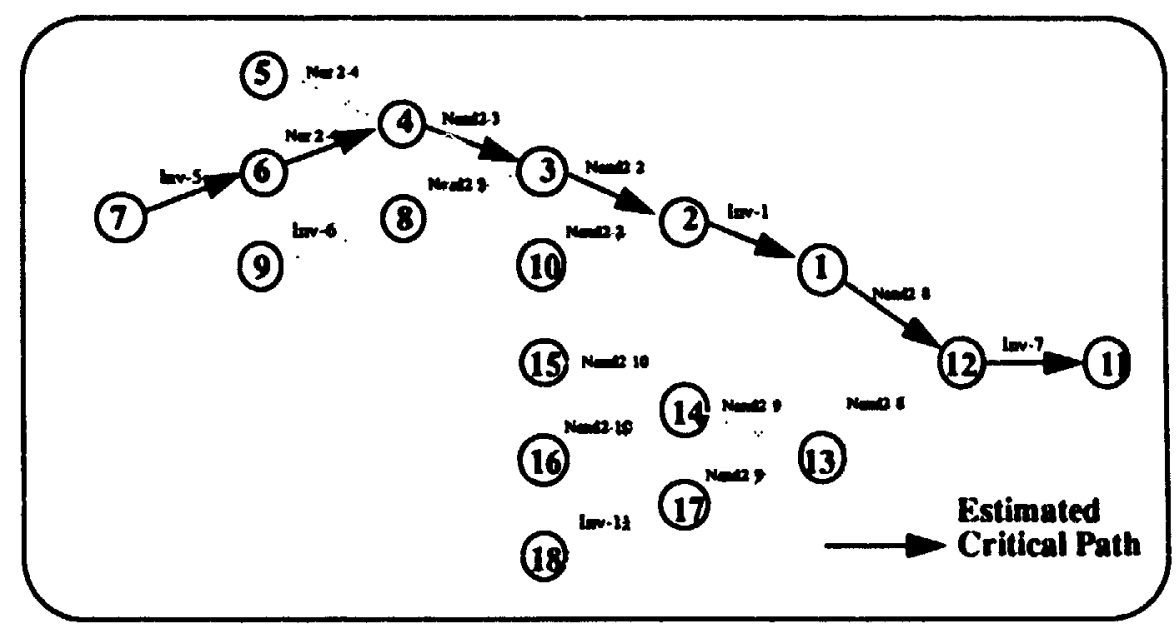

Eig.6.2 Network diagram for Example 1 with gate and node information.

When the optimization process terminates at a near optimum solution, the gate sizes and their corresponding actual areas are printed. Table 6.2 demonstrates the improvement in terms of the circuit area due to optimization. It shows the gates sizes and their actual area before and after the optimization.

A post processing step is applied after the optmizer solution is obtained. This is an automated adjustment if any of the gates is at its critical size. A gate is defined to be critical if the following expression holds:

$$
0 \leq\left(\text { size }-k \times S_{\text {critical }}\right) \leq 0.1 \quad \forall k=0,1,2, \ldots
$$

The value of $S_{\text {critical }}$ depends on the type of gate. This post processing step is required because the staircase area function is approximated by other functions. Final sizes for some of the gates could exceed the $S_{\text {critical }}$ values by a very small amount, but this could lead to a jump in the actual area because of the staircase behaviour. This step helps eliminating this extra area by reducing the sizes of those critical gates to slightly below their $S_{\text {critical }}$ values. In other words, by compromising with a little extra delay, the total area coald be reduced substantially.

However, this step does not necessarily provide a better area than the final 
solution obtained from the sptimizer. If too much delay is used in compensating for the area, the new solution would not be acceptable.

Table 6.ż: Optimization Result: Gates Information

\begin{tabular}{|c|c|c|c|c|c|c|}
\hline Gate & Gate & Fixed & Befor & Opt ${ }^{n}$ & After & Opt ${ }^{n}$ \\
\hline Number & Type & Area & Size & Area & Size & Area \\
\hline 1 & inv & 5.0 & 2.48 & 2.0 & 4.79 & 2.0 \\
\hline 2 & nand2 & 3.0 & 2.07 & 3.0 & 2.57 & 3.0 \\
\hline 3 & nand2 & 3.0 & 1.73 & 3.0 & 1.97 & 3.0 \\
\hline 4 & nor2 & 3.0 & 1.44 & 3.0 & 1.49 & 3.0 \\
\hline 5 & inv & 5.0 & 1.20 & 2.0 & 1.20 & 2.0 \\
\hline 6 & inv & 5.0 & 1.20 & 2.2 & 1.20 & 2.0 \\
\hline 7 & inv & 5.0 & 3.58 & 2.0 & 6.57 & 3.0 \\
\hline 8 & nand2 & 3.6 & 2.98 & 3.0 & 5.52 & 5.0 \\
\hline 9 & nand2 & 3.0 & $1.2 \mathrm{C}$ & 3.0 & 1.20 & 3.0 \\
\hline 10 & nand2 & 3.0 & 1.20 & 3.0 & 1.20 & 3.0 \\
\hline 11 & inv & 5.0 & 1.20 & 2.0 & 1.20 & 2.0 \\
\hline $\begin{array}{l}\text { Total } \\
\text { Area }\end{array}$ & & 43.0 & & 28.0 & & 31.0 \\
\hline Time & & $2.68 \mathrm{~ns}$ & & $2.65 \mathrm{~ns}$ & & $2.45 \mathrm{~ns}$ \\
\hline
\end{tabular}

It is shown by Table 6.2 that a trade-off between the speed aid the area of a circuit is often required to achieve a better performance circuic. The column under "Fixed Cell" indicates the result are obtained from using the Fixed Cell Library Approach. Other information is the latest_arrival_time for each node before and alter the optimization process. Table 6.3 show's this nodal information. 
Table 6.3: Optimization Results: Nodal Information

\begin{tabular}{|c|c|c|c|}
\hline Node Number & $\begin{array}{l}\text { LAT }{ }^{\mathbf{a}} \text { Before } \\
\text { Optimization }\end{array}$ & SLACK ${ }^{b}$ & $\begin{array}{l}\text { LAT After } \\
\text { Optimization }\end{array}$ \\
\hline \multicolumn{4}{|l|}{ Internal Nodes } \\
\hline 2 & 1.45 & 0 & 1.44 \\
\hline 3 & 1.13 & 0 & 1.06 \\
\hline 4 & 0.74 & 0 & 0.73 \\
\hline 5 & 0.22 & - & 0.21 \\
\hline 6 & 0.40 & 0 & 0.39 \\
\hline 7 & $0.2 i$ & - & 0.21 \\
\hline 8 & 0.83 & 1.15 & 0.40 \\
\hline 9 & 0.66 & - & 0.21 \\
\hline 10 & 0.26 & - & 0.25 \\
\hline 12 & 2.29 & 0 & 2.16 \\
\hline 13 & 0.91 & 2.45 & 1.25 \\
\hline 14 & 0.50 & 2.45 & 0.50 \\
\hline 15 & 0.21 & - & 0.21 \\
\hline 16 & 0.21 & - & 0.21 \\
\hline 17 & 0.39 & 2.8 & 0.39 \\
\hline 18 & 0.21 & - & 0.21 \\
\hline \multicolumn{4}{|l|}{ Output Nodes } \\
\hline 1 & 1.95 & 0 & 1.85 \\
\hline 11 & 2.65 & 0 & 2.45 \\
\hline
\end{tabular}

a. LAT stands for the Latest Arrival Time. 
b. This column shows the minimum slack (in terms of equivalent weight) that can be achieved by the nodes. A "-" means LAT does not apply to this nodi:

The SLACK column in Table 6.3 shows the estimated critical path for the circuit $(7 \rightarrow 6 \rightarrow 4 \rightarrow 3 \rightarrow 2 \rightarrow 1 \rightarrow 12 \rightarrow 11)$. The feasible initial solution, as described in Chapter 4, was estimated according to this path. Inspecting the last column of the table, which shows the latest arrival times of the nodes after optimization, observe that the actual critical path found afier optimization agrees with the estimated one before optimization. This result shows the effectiveness of the Relative Delay Estimate method in approximating the critical path of a circuit.

\subsection{Design Space Exploration \& Fixed Cell Library Approack.}

Circuit design often requires a trade-off between speed and total area. Higher speed usually means a bigger circuit and vice versa. An obvious example is the test problem demonstrated in the last section. Tables 6.2 and 6.3 show that the total area before optimization is greatly reduced by compromising with a relaxation on the timing requirement. This section uses the experimental results obtained in the last section and shows the effect on the total area of adjusting the speed, which is controlled by $T_{\max }$.

Theoretically, the shape of the trade-off curve should be similar to a negative exponential function, as shown in Fig.6.3, i.e. the smaller the delay, the larger the circuit area. 


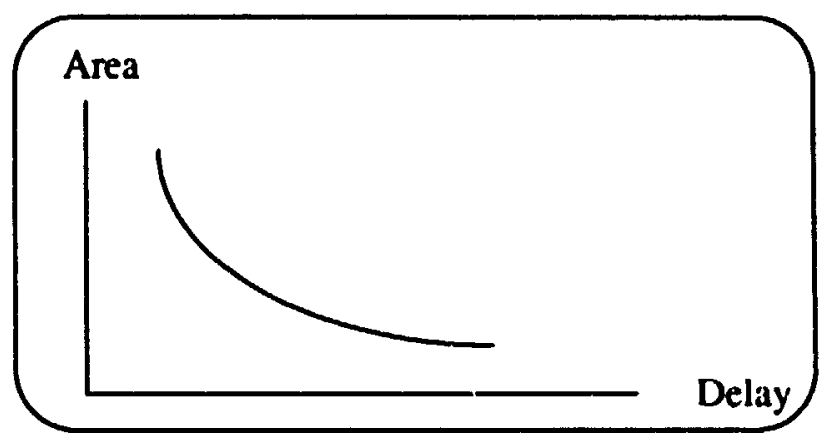

Eig. 6.3 Desired trade off between area and speed

The test problems used in this experiment are plotted within upper and lower bounds on the total delay (i.e. $T_{\max }$ ). The upper bound is the time such that any further increase in $\mathrm{T}_{\max }$ will not affect the final solution. That is, the final solution is the minimum set of sizes that can be achieved. The lower bound, on the other hand, is the time below which further decrease in $T_{\max }$ will render the problem infeasible, i.e. the minimum delay that the circuit can achieve. The curves are plutted using ten to twenty points of the actual area for both area models, and the points are generated within the upper and lower bounds of the delay. Fig. 6.4 and Fig.6.5 show the design trade-off between the area and the delay for Example 1 and Example 2, while Fig.6.6 and Fig.6.7 show the results for Example 3 and Example 4.

For each diagram, two set of points which represent the staircase and the linear area models are plotted. They are compared to show that whether the new staircase area model used in this thesis is worthy of application in the area of circuit design. 


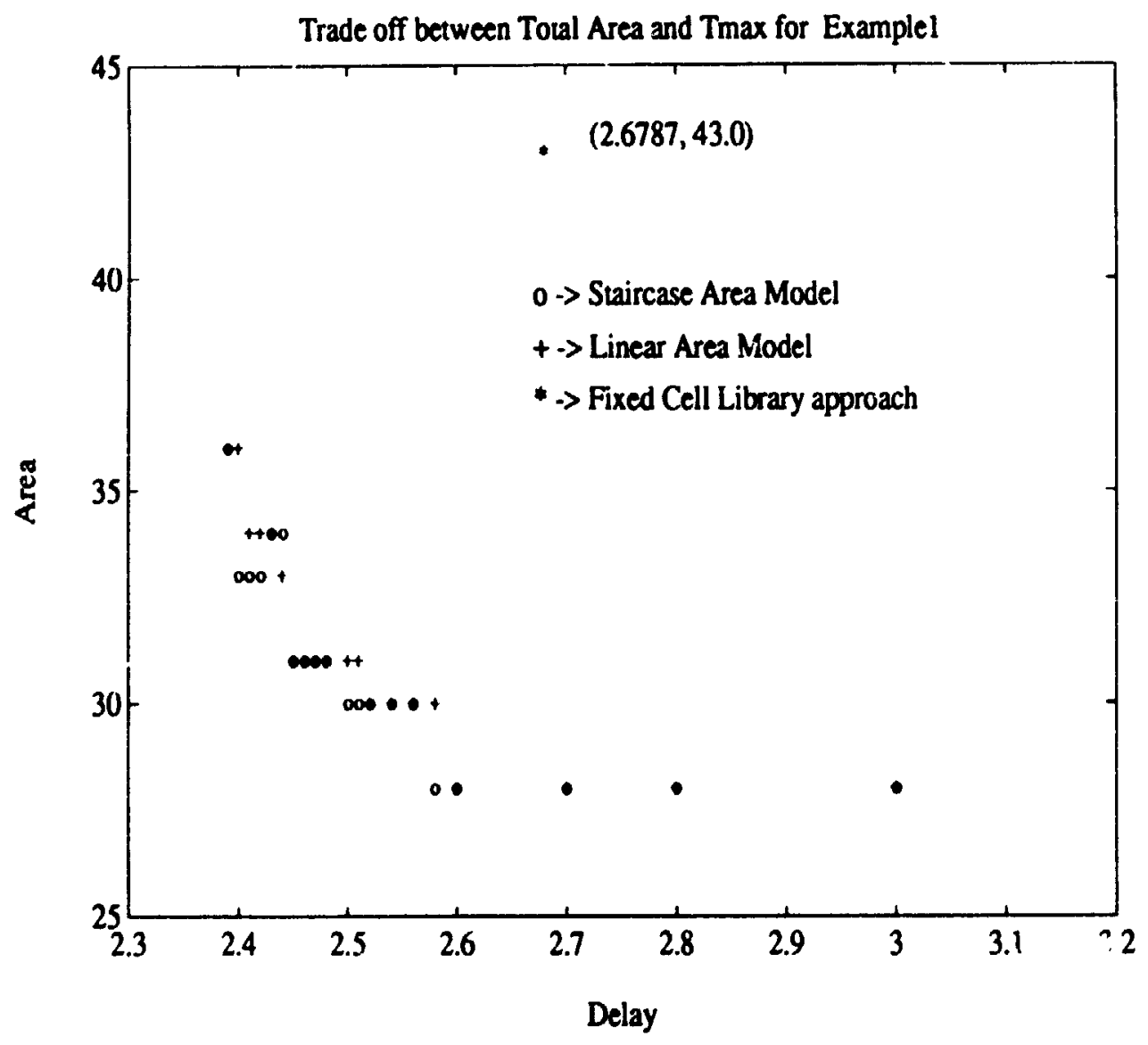

Fir.6.4 Trade-off between the total area and the speed for Example i.

In Fig.6.4, the results obtained from the staircase area model show a slight improvement over the linear model. Both area models agree with th expected trade-off relationship as shown by their curves. Similarly, the results found in Example 2 (Fig.6.5) are very close. The new staircase area model shows a very small improvement over different regions and even coincided with the results from the linear model in some other regions. Thr results from these two examples share two common phenomena: the results from both area models are close to each other and the new staircase area model is slightly better than the linear model. Since 
these two examples are small circuits which contain only a couple of gates, the choices for adjusting different gates for better results are limited. That is the reason why both examples show very close results. However, when compared with the result obtained from the Fixed Cell Library approach, both area models give substantially better results. In particular, the Time Area Product ${ }^{1}$ shows a $37 \%$ and $44 \%$ improvement by using the new staircase area model over the best result obtained by the Fixed Cell Library approach for Example 1 and Example 2 respectively.

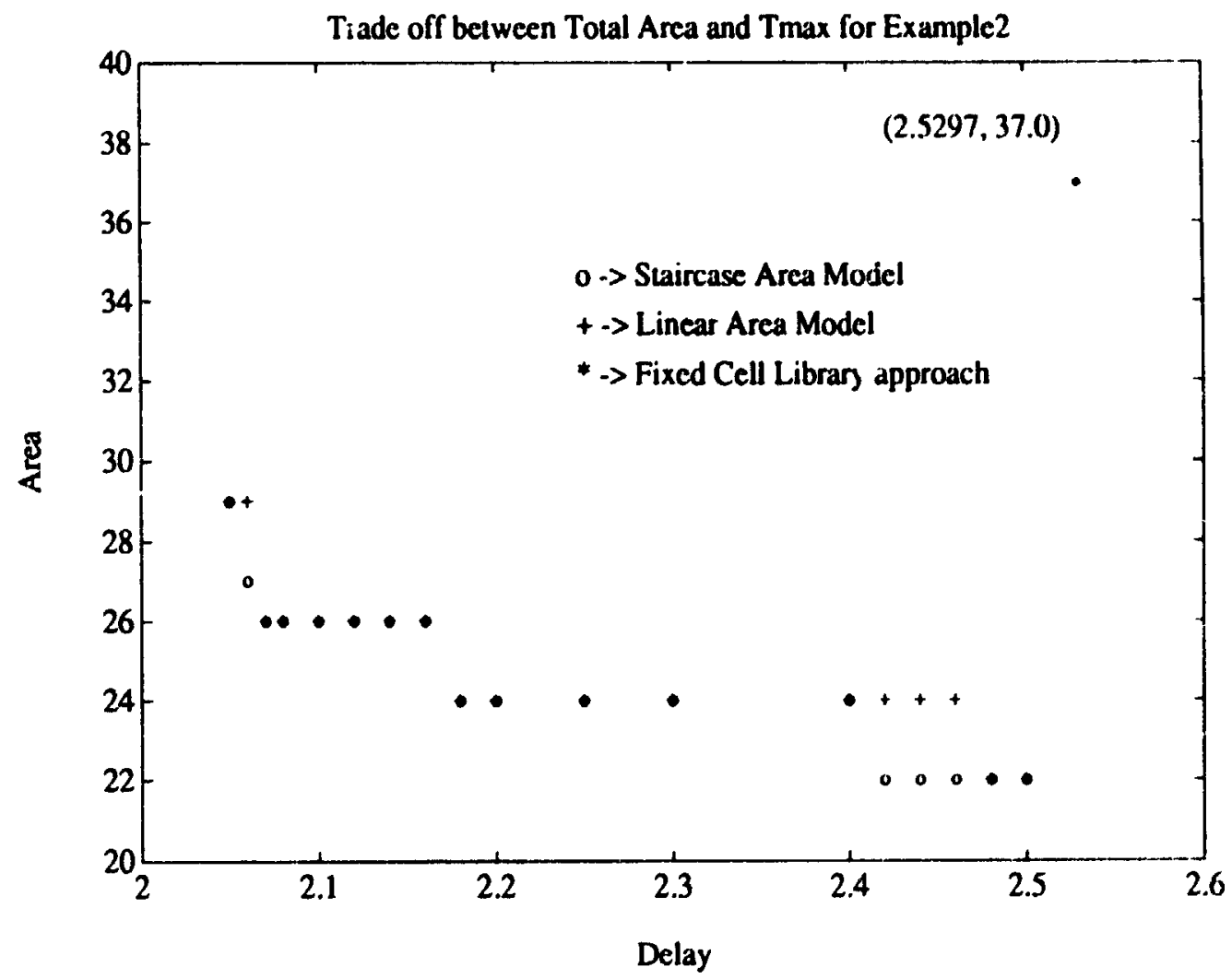

Eig.6.5 Trade-off between the total area and the speed for Example 2.

1. It is a product of time and area which is used for measuring the best possible combination of the results. 
For Example 3, where the results are shown in Fig.6.6, the results obtained from both area models are fairly close. The relationship between the area and the speed of the circuit is as expected. When compared with the Fixed Cell Library approach, the new staircase area model shows a $34 \%$ improvement in the Time Area Product.

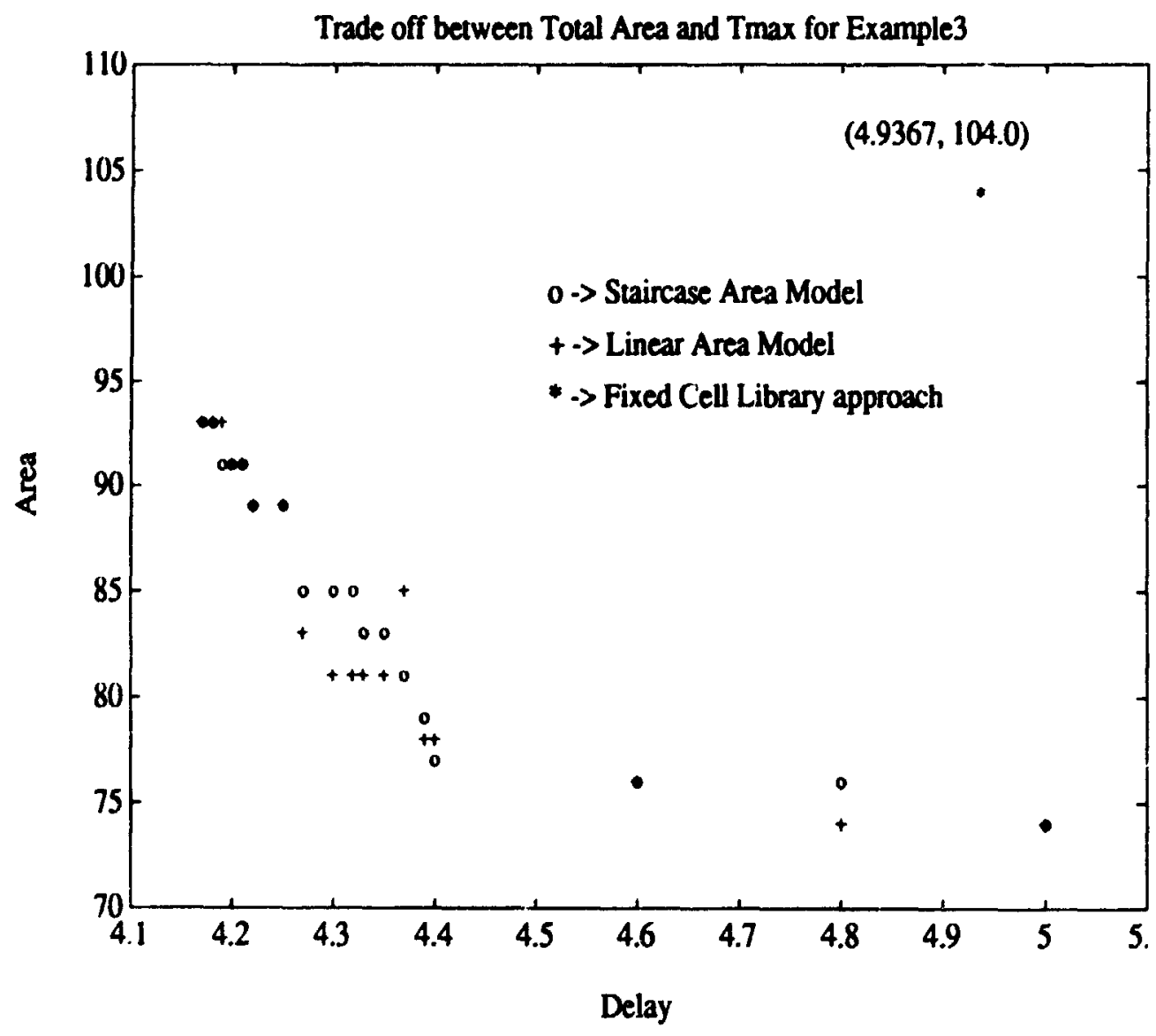

Fig.6.6 Trade-off between the total area and the speed for Example 3 .

Fig.6.7 shows the results obtained from Example 4. For this large circuit, the new area model shows a markedly better result than the linear area model. 
Since there are many cells in the circuit, the chance that the actual area of a cell is adjusted by the staircase function is increased. Moreover, the new area model shows a much better result than the Fixed Cell Library approach. Considering the Time Area Product for these models, the staircase model result is $31 \%$ better than the Fixed Cell Library result.

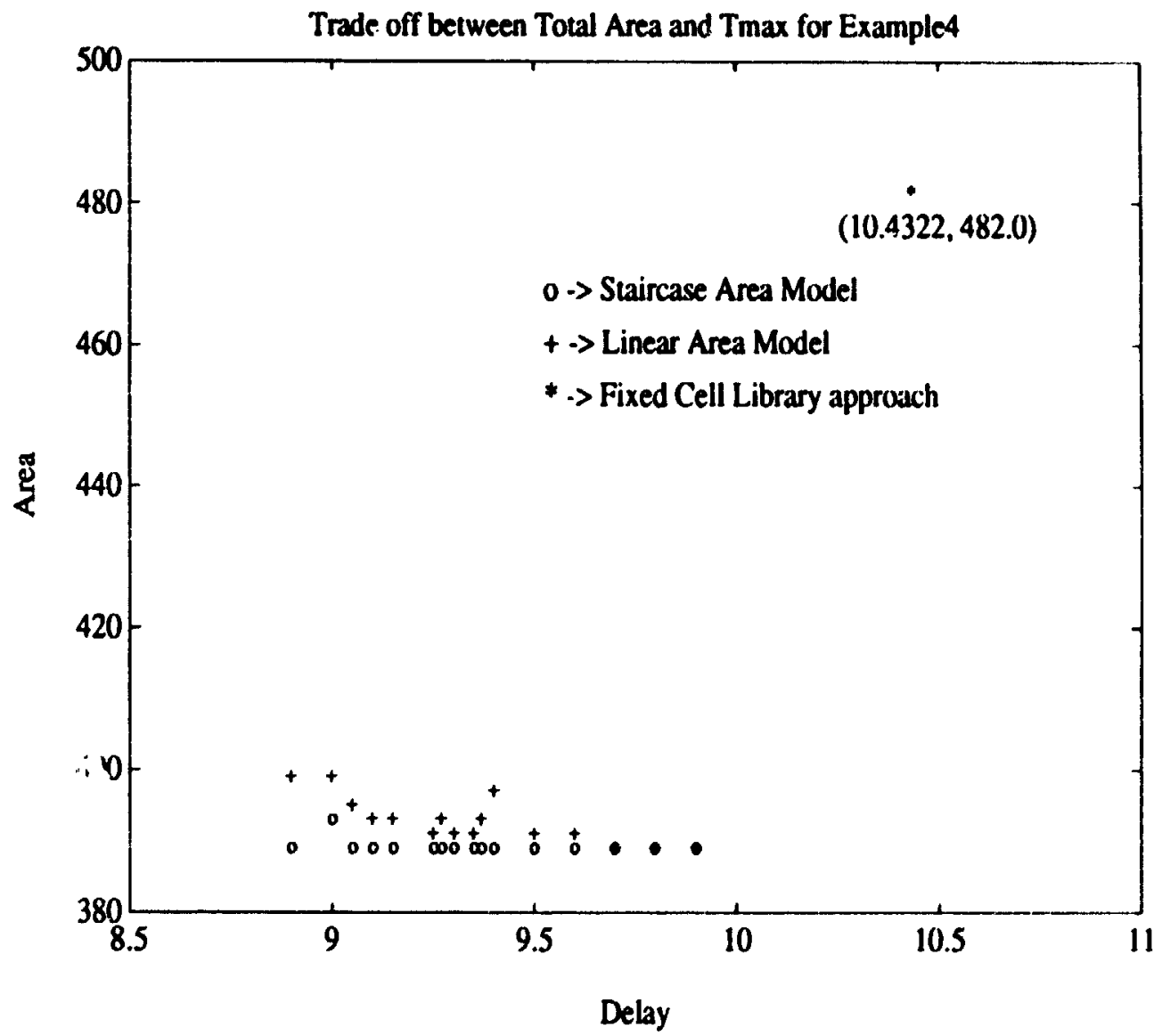

Fig.6.7 Trade-off between the total area and the speed for Example 4. 
Implementation and Experimr ital Results 79

\subsection{Chapter Summary}

The experiments lead directly to some conclusions. First, the new staircase area model is effective for circuit design, especially transistor sizing, although further research and adjustments are required. Second, this new area model is better in practice than some existing area models, the Linear area model and the Fixed Cell Library approach in particular. Finally, the integrated system including the new area models, the critical path estimation, the optimization, and the post processing, yield improved results on practical problems. 


\section{Chapter 7}

\section{Conclusions}

\subsection{Conclusions}

By observing the experimental results in the last chapter, the objectives of this thesis have been achieved. First, the new area and delay models have been integrated with a standard nonlinear optimizer in a system that is effective for the solution of the transistor sizing problem. The integrated system achieves better results than standard competing approaches, especially the fixed cell library approach. Second, an effective algorithm for providing an initial set of transistor sizes to the nonlinear optimizer has been developed and demonstrated. It prevents stalling of the optimizer at local infeasibilities. Moreover, the integrated system combining the area models and the general nonlinear optimizer provides the expected relationship between total area and maximum delay.

\subsection{Contributions}

This thesis has made several contributions in the area of transistor sizing. First of all, this thesis demonstrates a practical system giving improved resuits for the transistor sizing problem, as compared to standard methods such as a fixed cell library approach. Also, the effectiveness of standard general nonlinear optimization methods for the solution of the transistor sizing problem is demonstrated. Of course, the optimization is closely tied to the area and delay models which are used. Third, an effective critical path estimator ard initial transistor sizing heuristic are developed. The initial sizing heuristic could in principle be used by itself to 
solve the sizing problem, but is better used to provide an initial point to the general nonlinear optimizer. This arrives at a better final result. Moreover, the two area models (based on previous work) are presented and their effectiveness in solving the transistor sizing problem (in conjunction with a general nonlinear optimizer) is demonstrated. The last contribution is to provide a brief study of the relationship between the total area and the maximum delay in fifierent circuits.

\subsection{Recommendations for Future Work}

The area models are the most important candidates for further work. In this thesis, the new area model is approximated by piecewise curves, which accounts for the inaccuracy of the results. Ain improvement can be made by applying the new area model directly in :ne software prototype. New subroutines should then be developed to handle the non-differentiability of the area model.

There are two more minor adjustments that could be made. The relative delay estimates for estimating the critical path can be improved by a more detailed statistical experimental setup. In this thesis, the environments, which include the input switching time, the current gate size and the gate output capacitive load, are set up at random within ranges to ensure validity. However, the behaviour of different types of gates may also be affected by some correlations among the factors described above, or even by other elements that have not been considered here. Hence, a careful study of the gate behaviour by statistical experiments may improve the accuracy of the estimated critical path in general.

The last adjustment can be made in the algorithm that assigns the initial set of sizes on the estimated critical path. Recall that the algorithm used in this thesis is based on sizing a chain of inverters, that is, the gate sizes on the estimated critical path are assumed to increase according to a pre-specified function. However, 
this may not be true for other types of gates. Thus, a study of the behaviour of different types of gates in a chain may provide a better sizing algorithm, yielding a better feasible initial solution. 


\section{Appendix A. Glossary of Terms and Symbols}

amplifier

$\mathrm{C}_{\mathrm{gs}}, \mathrm{C}_{\mathrm{gd}}$

CMOS

complex gate

divide-and-conquer

GND

$I_{D S}$

MOS

nondifferentiable

penalty function

pull-up/pull-down

RC

$\mathrm{V}_{\mathrm{DS}}$
A circuit uses to increase or magnify a very weak signal until it is capable of controlling sufficient electronic power.

Gate-to-source capacitance and Gate-to-drain capacitance.

Complementary Metal Oxide Semiconductor.

A combinatorial logic structure, which performs alternating Boolean "AND" and "OR" functions ending in an "INVERT" in a CMOS implementa. tion.

A strategy in problem-solving techniques by partirioning the original problem into sub-problems and gethered to get the final solution.

Ground voltage, i.e. $0 \mathrm{~V}$.

Drain-to-source current.

Metal-Oxide-Silicon

Mathematical term, uses to describe a function with non-existing limit(s) within a certain range.

A mathematical function used in optimization. It prevents the directional search from going out of the feasible region.

Terms to describe whether a device (or a series of devices) is connected so that the output voltage is being brought High/Low.

Resistance-Capacitance.

Drain-to-source voltage. 
Appendix A. Glossary of Terms and Symbols it

$\mathrm{V}_{\mathrm{IH}}, \mathrm{V}_{\mathrm{IL}}$

$\mathrm{V}_{\mathrm{OH}}, \mathrm{v}_{\mathrm{OL}}$

VLSI

High and low input voltage.

High and low output voltage.

Very Large Scale Integrated. 

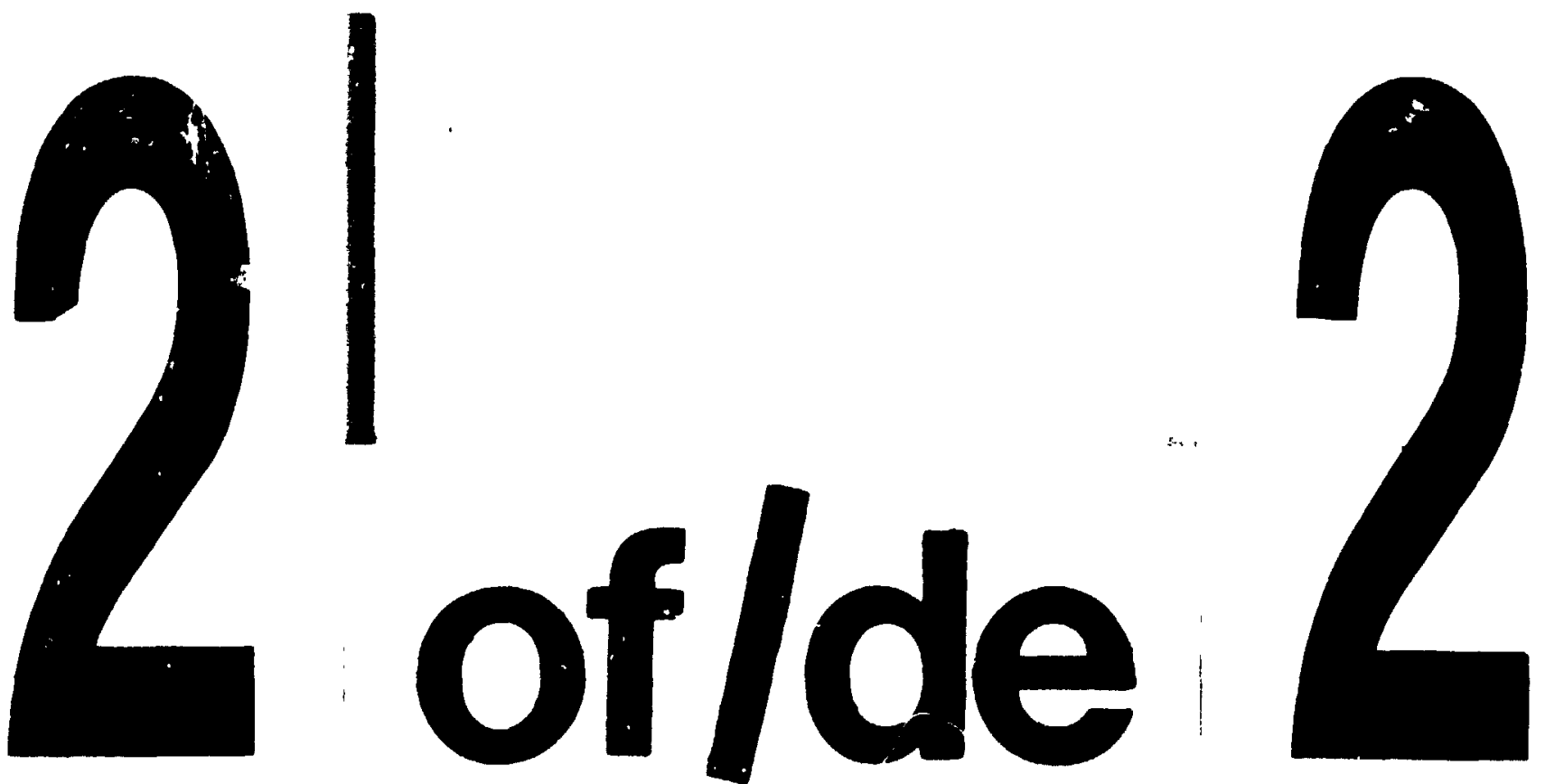

PM-1 31/2" $\times 4$ " PHOTOGRAPHIC MICROCOPY TARGET NBS $1010 a$ ANSI/ISO *2 EOUIVALENT

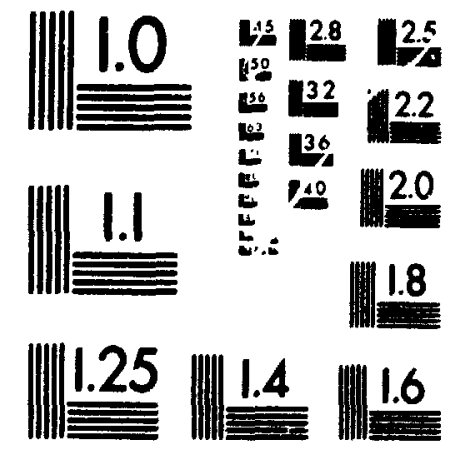

PRECISION"W RESOLUTION TARGETS

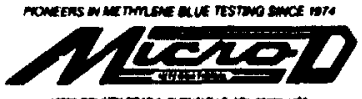




\section{Appendix B. User Manual}

The circuit optimization program described in this thesis can be invoked under a SUN workstation by calling "GLTSP". The following is the main menu and the job description correspond to each option.

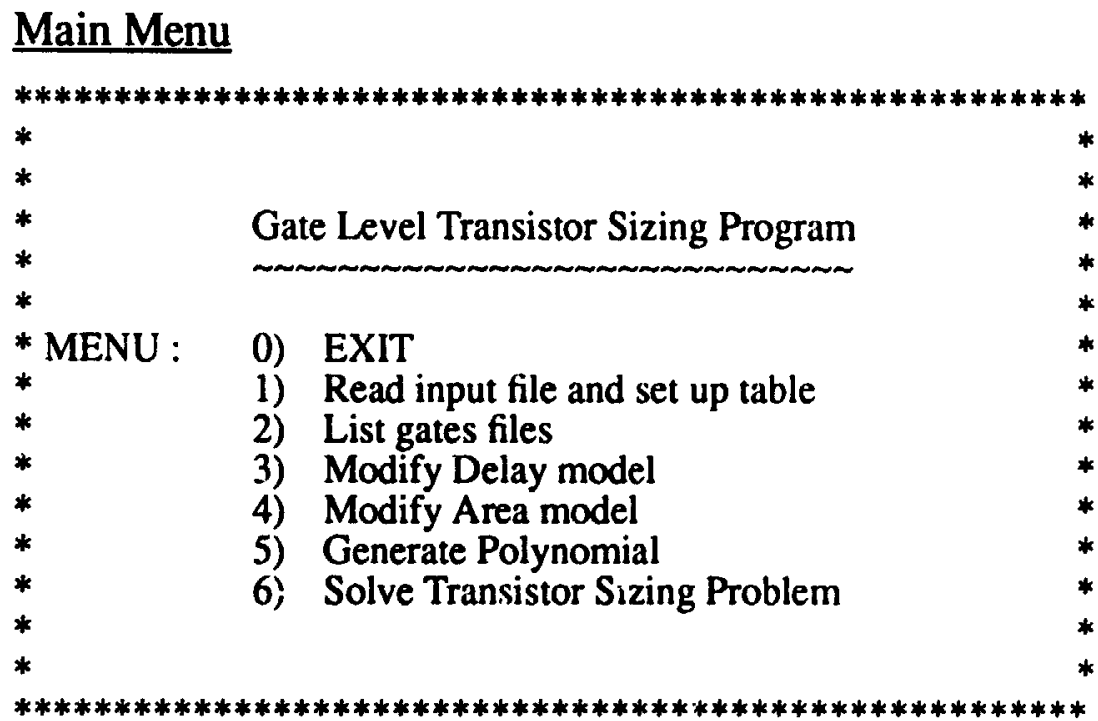

Choice:

Menu description:

Exit

Terminate the execution of the program.

1 Reading Input file and Set up table

This option allows the user to input the files to be read by 
the program. The following questions will be asked:

1. Please input the name of the circur file:

2. Please input the name of gates file:

Gates file is asked in the case that if more than one delay model is used, the user only need to specify the delay model and do not have to adjust the information for the gates for different models repeatedly.

The table uses to store the information is created at this time according to the circuit file.

\section{List Gates Files}

Each delay model corresponds to one single gates file. If more than one delay model are used, the list of delay models can be found by choosing this option. Gates files are standardized with extension “.cir". Thus, before choosing option 1, this option can be used to figure out which delay model is available.

\section{Modify Delay model}

This option allows the user to modify any existing delay model, or creates one by entering a new name to the following question:

Please enter the name of the gates file: (.cir)

Press 1) Add gate 2) Modify gate 3) EXIT 


\section{OPTION 1: Add gate}

This option lets the user adding a new type of gate to the gates file. The following question is asked:

How many terms for this gate:

Coefficient:

Expression:

Coefficient and Expression are prompted according to the number of terms for the gate.

OPTION 2: Change gate

This option allows the user to change the information of a gate. First, it prompts for the name of the gate:

Name of the gate to be modified:

If the name exists in the file, it will ask for the particular term to change.

Indicate which term to change:

Press 1) Coefficient 2) Expression 3) Both

4

Modify Area model

This command allows the user to change the area model for any type of gate. The following questions are asked:

Please enter the name of the input data file:

Please enter the name of the output coordinate file:

Then, enter the new $x$-coordinates in one line and the same for the $y$-coordinates. 


\section{Getierate Polynomial}

This option will generate the piecewise polynomial to approximate the staircase area function for different types of gates. The following question is asked:

Please enter the type of gate to generate:

In order to adjust the shape of the approximated curve, an user adjustable parameter, $\alpha(0<\alpha<1)$, is used for experimentation. The following question is asked until the user is satisf.ed with the shape of the approximated curve:

Please enter a value for $\alpha$ (in decimal):

The results are both the piecewise polynomials's coefficients and a graphical feedback on the shape of the curve comparing with the original staircase function. The graphical result can be obtained by first invoking MATLAB, changing the directory to "tsp", and then calls the file with "co_TYPE.m", where TYPE corresponds to the type of the gate.

6 Solve Transistor Sizing Problem

This option links all the information together and solves the corresponding transistor sizing problem. Final decision can be made here whether any adjustment is needed:

Press 0) for EXIT I) for starting NLP program

If option 1 is chosen, the following question is asked: 
Maximum Delay Time:

Output Capacitive Load:

Please input the title:

Title is any name for :nis particular problem. Time is speci-

fied in $\mu \mathrm{s}$ and capacitive load is in $\mathrm{pF}$.

Option 0 is chosen if the user wants to modify previous information. 


\section{Appendix C. Circuit files and the results}

Testing problem \#1: 5 primary inputs. 2 primary outputs and 11 gates.

model test

inputs a bc de

.outputs $f g$

gate $! a=f_{\text {nnot }} O=f$

.gate (ab)' $a=m+6 b=a O=f_{-}$not

.gate ! $a=b \quad O=b \_n o t 0$

.gate (ab)' $a=m+5 b=b \_n o t 0 ~ O=m+6$

gate ! $a=c 0=c_{-}$not 0

gate $(a+b)^{\prime} a=e b=c \_$rot $00=m t 5$

.gate ! $a=g_{-}$not $O=g$

gate (ab)' $a=f b=m+13 O=g \_n o t$

gate ! $a=d \mathrm{O}=d \_$not0

.gate $(a b)^{\prime} a=m t 12 b=d \_n o t 00=m t 13$

gate (ab)' $a=e b=a O=m t 12$

.end

Testing problem \#2: 4 primary inputs, 2 primary outputs and 9 gates.

.model non-tree structure for testing

inputs a bcd

.outputs $f g$

.gate $! a=[60] O=f$

-gate (ab) $a=[30] b=[50] 0=[60]$

.gate !a $a=[20] \mathrm{O}=[30]$

.gate (ab)' $a=a b=[10] O=[20]$

gate ! $a=b \quad O=[10]$

gate $(a b)^{\prime} a=[10] b=[40] O=[50]$

gate ! $a=c \mathrm{O}=[40]$

.gate $(a b)^{\prime} a=f b=[70] O=g$

gate ! $a=d O=[70]$

.end 
Testing problem *3:5 primary inputs. 3 primary outputs and 28 aates.

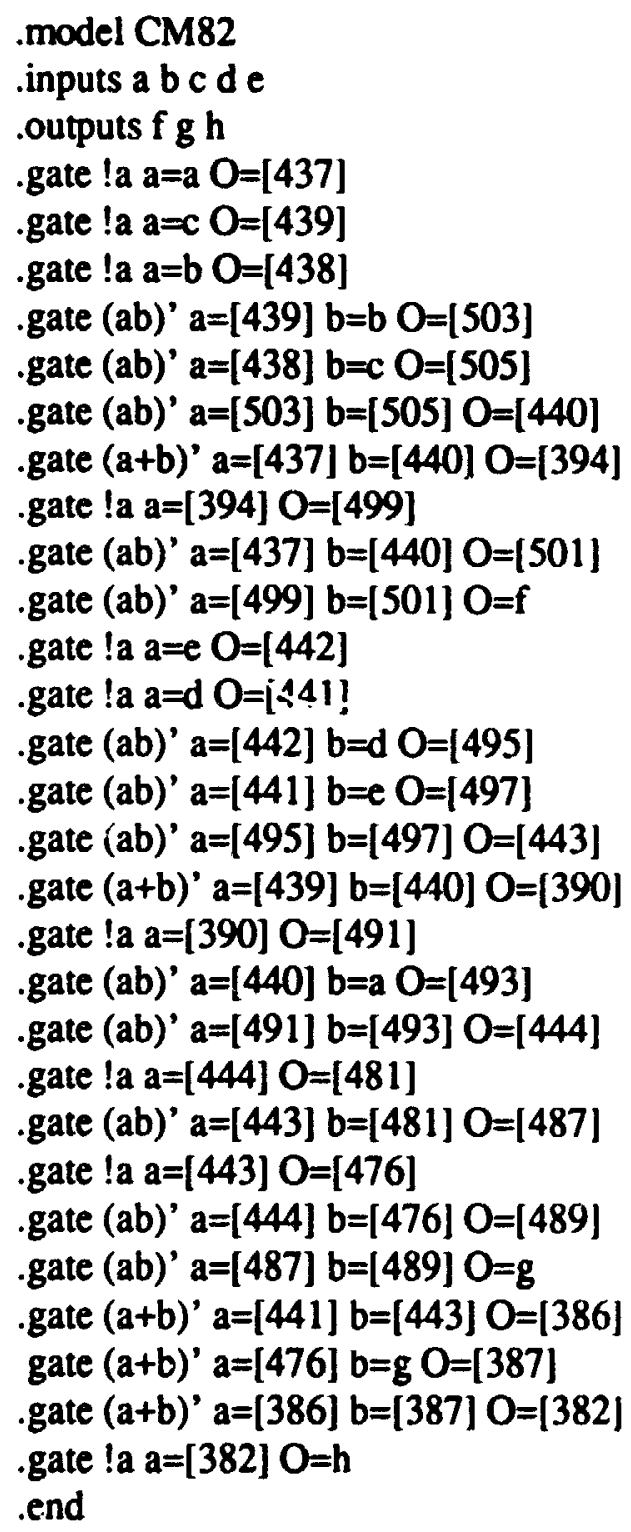


Testinp problem *4: 36 primary inputs, 16 primary outputs and 136 gates. model count

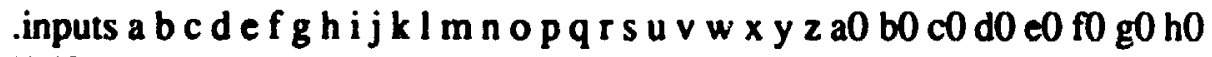
i0 j0 .outputs $\mathrm{k} 010 \mathrm{m0} \mathrm{n} 0 \mathrm{o} 0 \mathrm{p} 0 \mathrm{q} 0 \mathrm{r} 0 \mathrm{~s} 0 \mathrm{t} 0 \mathrm{u} 0 \mathrm{v} 0 \mathrm{w0} \times 0 \mathrm{y} 0 \mathrm{z} 0$ .gate !a a=s $O=[588]$ .gate !a $a=r O=[457]$ .gate ! $a=u \quad O=[458]$ .gate (ab)' $a=[457] b=[458] O=[459]$ gate (ab)' $a=r \quad b=u \quad O=[857]$ .gate (ab) $a=[459] b=[857] 0=[719]$ .gate $\mathrm{a} a \mathrm{a}=\mathrm{q} \mathrm{O}=[456]$ .gate (ab)' $a=[719] b=q O=[853]$ gate !a $a=p \quad O=[535]$ .gate (ab)' $a=[535] b=[456] O=[855]$ .gate $(a b c)^{\prime} a=[588] b=[853] c=[855] 0=k 0$ .gate $(a+b)^{\prime} a=v b=[459] 0=[78]$ .gate ! $a \mathrm{a}=[78] \mathrm{O}=[462]$ .gate (ab) $a=[459] b=v O=[851]$ .gate (ab)' $a=[462] b=[851] \mathrm{O}=[721]$ .gate (ab)' $a=[721] b=q O=[847]$ -gate ! $a=0 \quad O=[533]$ -gaie (ab)' $a=[533] b=[456] \quad O=[849]$ .gate $(a b c)^{\prime} a=[588] b=[847] c=[849] \quad O=10$ .gate ! $a=w O=[464]$ .gate (ab)' $a=[464] b=[78] O=[465]$ .gate $(a b)^{\prime} a=[462] b=w \quad O=[845]$ -gate (ab)' $a=[465] b=[845] \mathrm{O}=[723]$ .gate (ab)' $a=[723] b=q \quad O=[841]$ -gate ! $a=n \mathrm{O}=[531]$ -gate (ab)' $a=[531] b=[456] O=[843]$ -gate $(a b c)^{\prime} a=[588] b=[841] c=[843] \quad O=m 0$ -gate $(a+b)^{\prime} a=x \quad b=[465] 0=y 1$ .gate ! $a=y 10=[468]$ .gate (ab) $a=[465] b=x \quad O=[839]$

.gate (ab)' $a=[468] b=[839] 0=[725]$ gate (ab)' $a=[725] b=q O=[835]$ gate ! $a=m ~ O=[529]$ -gate (ab)' $a=[529] b=[456] 0=[837]$ 


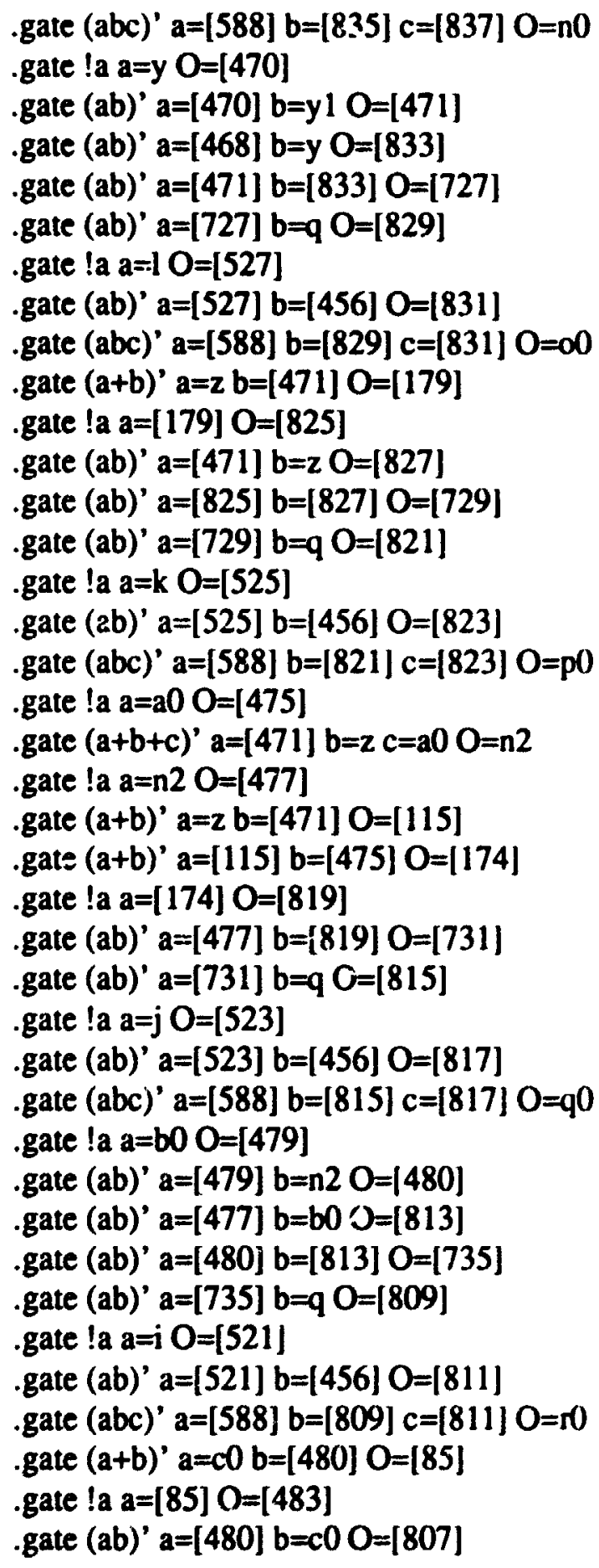




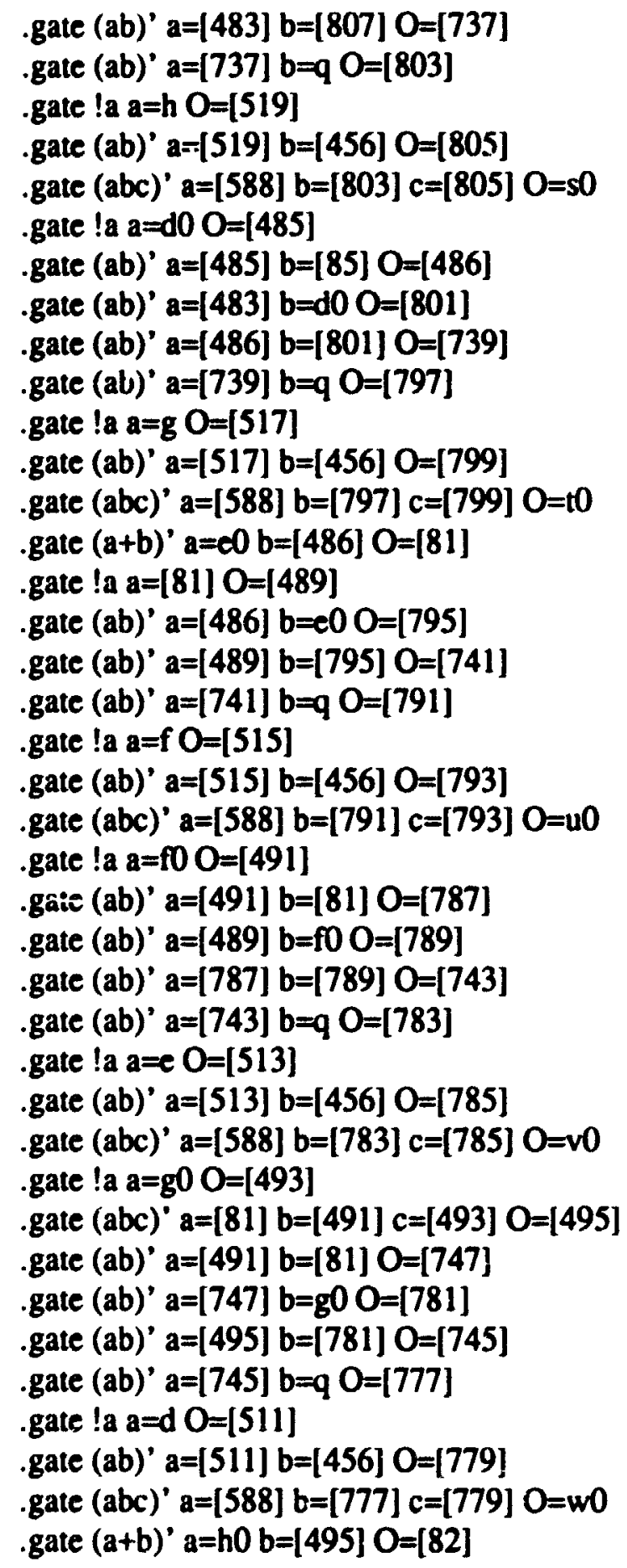




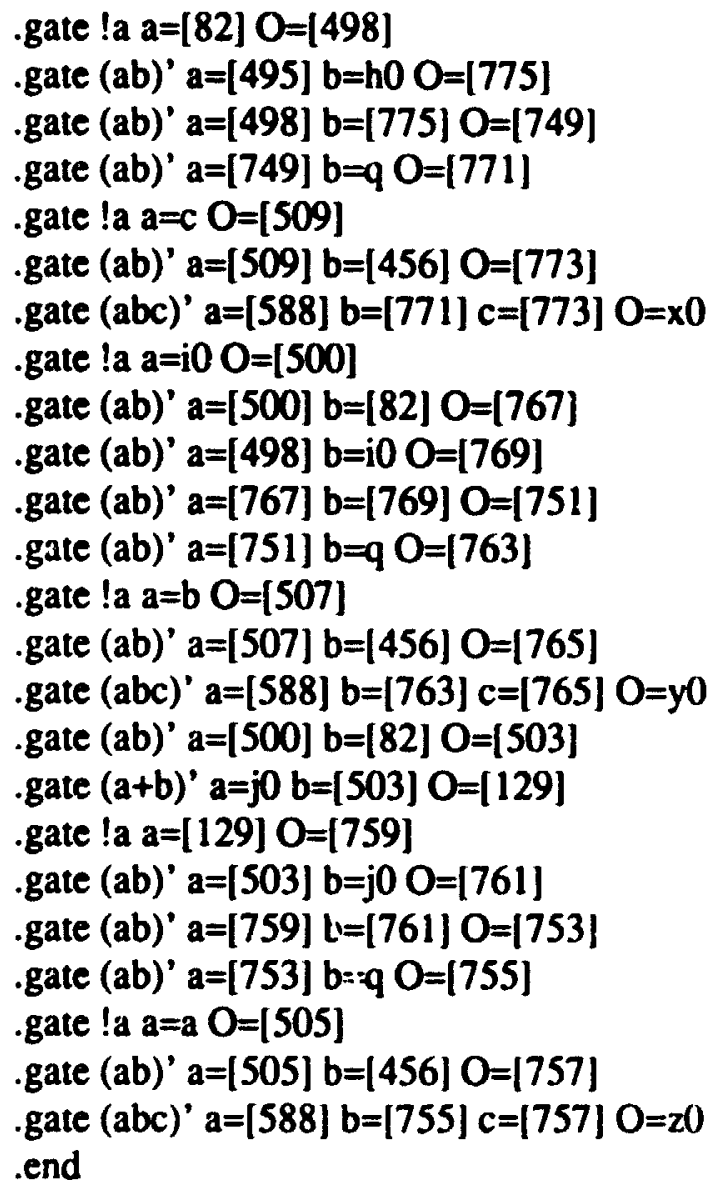


Problem Title: Example 1

\begin{tabular}{|c||c|c||c|c|}
\hline$T_{\max }$ & Area(Staircase) & Time*Area & Area(Linear) & Time*Area \\
\hline \hline 2.39 & 36.0 & 86.04 & 36.0 & 86.04 \\
\hline 2.40 & 33.0 & 79.20 & 36.0 & 86.40 \\
\hline 2.41 & 33.0 & 79.53 & 34.0 & 81.94 \\
\hline 2.42 & 33.0 & 79.86 & 34.0 & 82.28 \\
\hline 2.43 & 34.0 & 82.62 & 34.0 & 82.62 \\
\hline 2.44 & 34.0 & 82.96 & 33.0 & 80.52 \\
\hline 2.45 & 31.0 & 75.95 & 31.0 & 75.95 \\
\hline 2.46 & 31.0 & 76.26 & 31.0 & 76.26 \\
\hline 2.47 & 31.0 & 76.57 & 31.0 & 76.57 \\
\hline 2.48 & 31.0 & 76.88 & 31.0 & 76.88 \\
\hline 2.50 & 30.0 & 75.00 & 31.0 & 77.50 \\
\hline 2.51 & 30.0 & 75.30 & 31.0 & 77.81 \\
\hline 2.52 & 30.0 & 75.60 & 30.0 & 75.60 \\
\hline 2.54 & 30.0 & 76.20 & 30.0 & 76.20 \\
\hline 2.56 & 30.0 & 76.80 & 30.0 & 76.80 \\
\hline 2.58 & 28.0 & 72.24 & 30.0 & 77.40 \\
\hline 2.60 & 28.0 & 72.80 & 28.0 & 72.80 \\
\hline 2.70 & 28.0 & 75.60 & 28.0 & 75.60 \\
\hline 2.80 & 28.0 & 78.40 & 28.0 & 78.40 \\
\hline 3.00 & 28.0 & 84.00 & 28.0 & 84.00 \\
\hline
\end{tabular}

Eixed Acea Appro:ach

Area: 43.0 
Problem Title: Example 2

\begin{tabular}{|c||c|c||c|c|}
\hline$T_{\max }$ & Area (Staircase) & Time x Area & Area (Linear) & Time x Area \\
\hline \hline 2.05 & 29.0 & 59.45 & 29.0 & 59.45 \\
\hline 2.06 & 27.0 & 55.62 & 29.0 & 59.74 \\
\hline 2.07 & 26.0 & 53.82 & 26.0 & 53.82 \\
\hline 2.08 & 26.0 & 54.08 & 26.0 & 54.08 \\
\hline 2.10 & 26.0 & 54.60 & 26.0 & 54.60 \\
\hline 2.12 & 26.0 & 55.12 & 26.0 & 55.12 \\
\hline 2.14 & 26.0 & 55.64 & 26.0 & 55.64 \\
\hline 2.16 & 26.0 & 56.16 & 26.0 & 56.16 \\
\hline 2.18 & 24.0 & 52.32 & 24.0 & 52.32 \\
\hline 2.20 & 24.0 & 52.80 & 24.0 & 52.80 \\
\hline 2.25 & 24.0 & 54.00 & 24.0 & 54.00 \\
\hline 2.30 & 24.0 & 55.20 & 24.0 & 55.20 \\
\hline 2.35 & 24.0 & 56.40 & 24.0 & 56.40 \\
\hline 2.40 & 24.0 & 57.60 & 24.0 & 57.60 \\
\hline 2.42 & 22.0 & 53.24 & 24.0 & 58.08 \\
\hline 2.44 & 22.0 & 53.68 & 24.0 & 58.56 \\
\hline 2.46 & 22.0 & 54.12 & 24.0 & 59.04 \\
\hline 2.48 & 22.0 & 54.56 & 22.0 & 54.56 \\
\hline 2.50 & 22.0 & 55.00 & 22.0 & 55.00 \\
\hline
\end{tabular}

Eixed Area Approach

Area: 37.0

Time: 2.5297

Product(Time x Area): 93.5989 


\section{Problem Title: Example 3}

\begin{tabular}{|c||c|c||c|c|}
\hline$T_{\max }$ & Area (Staircase) & Time x Area & Area (Linear) & Time x Area \\
\hline \hline 4.17 & 93.0 & 387.81 & 93.0 & 387.81 \\
\hline 4.18 & 93.0 & 388.74 & 93.0 & 388.74 \\
\hline 4.19 & 91.0 & 381.29 & 93.0 & 389.67 \\
\hline 4.20 & 91.0 & 382.20 & 91.0 & 382.20 \\
\hline 4.21 & 91.0 & 383.11 & 91.0 & 383.11 \\
\hline 4.22 & 89.0 & 375.58 & 89.0 & 375.58 \\
\hline 4.25 & 89.0 & 378.25 & 89.0 & 378.25 \\
\hline 4.27 & 85.0 & 362.95 & 83.0 & 354.41 \\
\hline 4.30 & 85.0 & 365.50 & 81.0 & 348.30 \\
\hline 4.32 & $(87.0) 85.0^{2}$ & 367.20 & 81.0 & 349.92 \\
\hline 4.33 & $(85.0) 83.0$ & 359.39 & 81.0 & 350.73 \\
\hline 4.35 & 83.0 & 361.05 & 81.0 & 352.35 \\
\hline 4.37 & 81.0 & 85.0 & 353.97 & 371.45 \\
\hline 4.39 & 79.0 & 346.81 & 78.0 & 342.42 \\
\hline 4.40 & 77.0 & 338.80 & 78.0 & 343.20 \\
\hline 4.60 & 76.0 & 349.60 & 76.0 & 349.60 \\
\hline 4.80 & 76.0 & 364.80 & 74.0 & 355.20 \\
\hline 5.0 & 74.0 & 370.00 & 74.0 & 370.00 \\
\hline
\end{tabular}

a. The value within the bracket shows the sclution before the post processing step.

Eixed Area Approach

$\begin{array}{lll}\text { Area: } 104.0 & \text { Time: } 4.9307 & \text { Product(Time } \times \text { Area): } 512.79\end{array}$ 


\section{Problem Title: Example 4}

\begin{tabular}{|c||c|c||c|c|}
\hline$T_{\max }$ & Area (Staircase) & Time x Area & Area (Linear) & Time x Area \\
\hline \hline 8.90 & 389.0 & 3462.10 & 399.0 & 3551.10 \\
\hline 9.00 & 393.0 & 3537.00 & 399.0 & 3591.00 \\
\hline 9.05 & 389.0 & 3520.45 & 395.0 & 3574.75 \\
\hline 9.10 & 389.0 & 3539.90 & $* 393.0^{\mathrm{a}}$ & 3576.30 \\
\hline 9.15 & 389.0 & 3559.35 & $* 393.0$ & 3595.95 \\
\hline 9.25 & 389.0 & 3598.25 & $* 391.0$ & 3616.75 \\
\hline 9.27 & 389.0 & 3606.03 & $* 393.0$ & 3643.11 \\
\hline 9.30 & 389.0 & 3617.70 & $* 391.0$ & 3636.30 \\
\hline 9.35 & 389.0 & 3637.15 & $* 391.0$ & 3655.85 \\
\hline 9.37 & 389.0 & 3644.93 & $* 393.0$ & 3682.41 \\
\hline 9.40 & 389.0 & 3656.60 & $* 397.0$ & 3731.80 \\
\hline 9.50 & 389.0 & 3695.50 & $* 391.0$ & 3714.50 \\
\hline 9.60 & 389.0 & 3734.40 & $* 391.0$ & 3753.60 \\
\hline 9.70 & 389.0 & 3773.30 & $* 389.0$ & 3773.30 \\
\hline 9.80 & 389.0 & 3812.20 & $* 389.0$ & 3812.20 \\
\hline 9.90 & 389.0 & 3851.10 & $* 391.0$ & 3870.90 \\
\hline 10.0 & $* 389.0$ & 3890.00 & $* 391.0$ & 3910.00 \\
\hline 9.10 & 300 & & \\
\hline
\end{tabular}

a. A * before the solution means that the optimizer is terminated with the Kuhn-Tucker conditions satisfied within 1.0E-4 at the final point.

\section{Eixed Area Approach}

Area: 482.0

Time: 10.4322

Product(Time x Area): 5028.32 


\section{Appendix D. Sample Fortran subroutines codes}

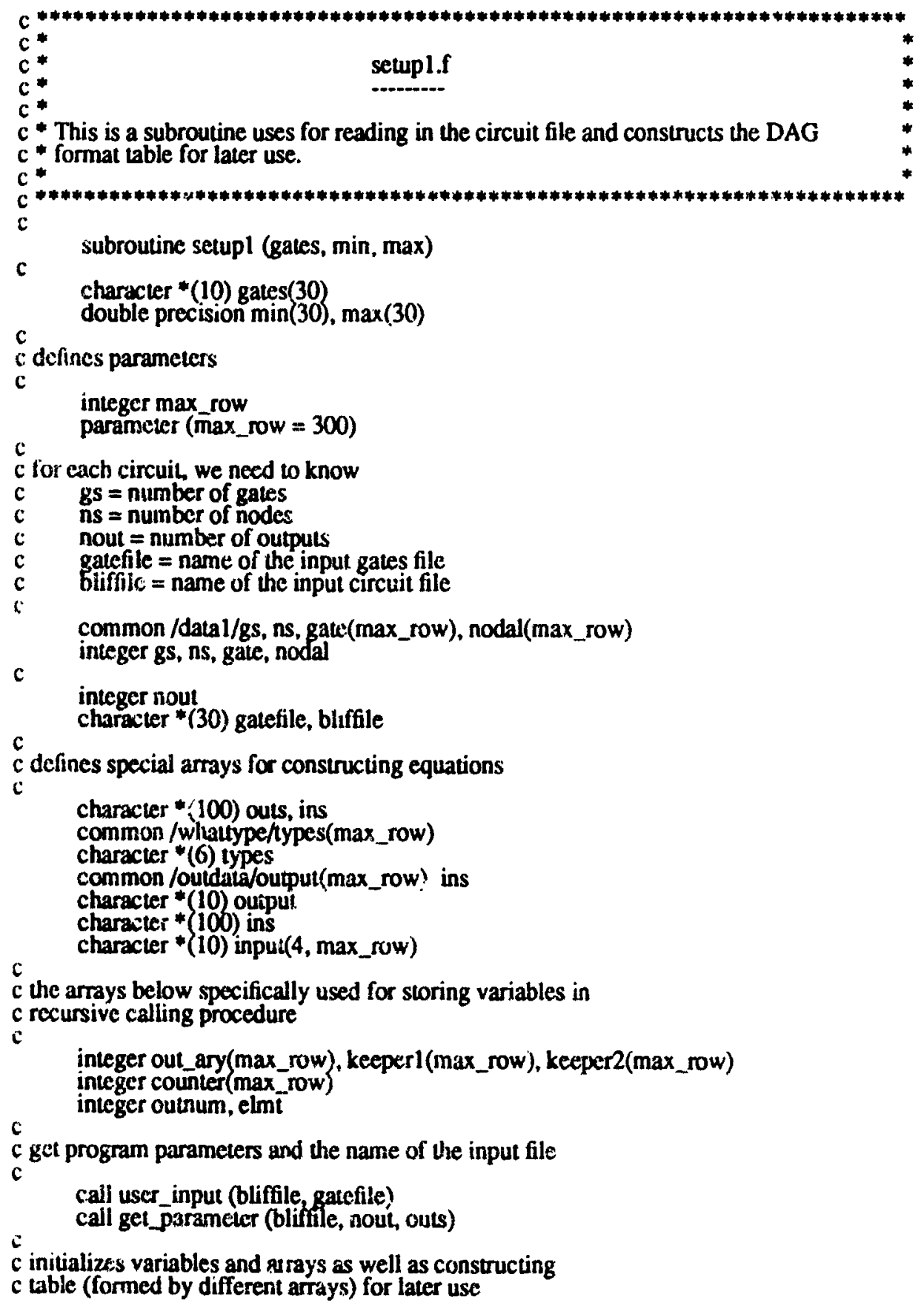




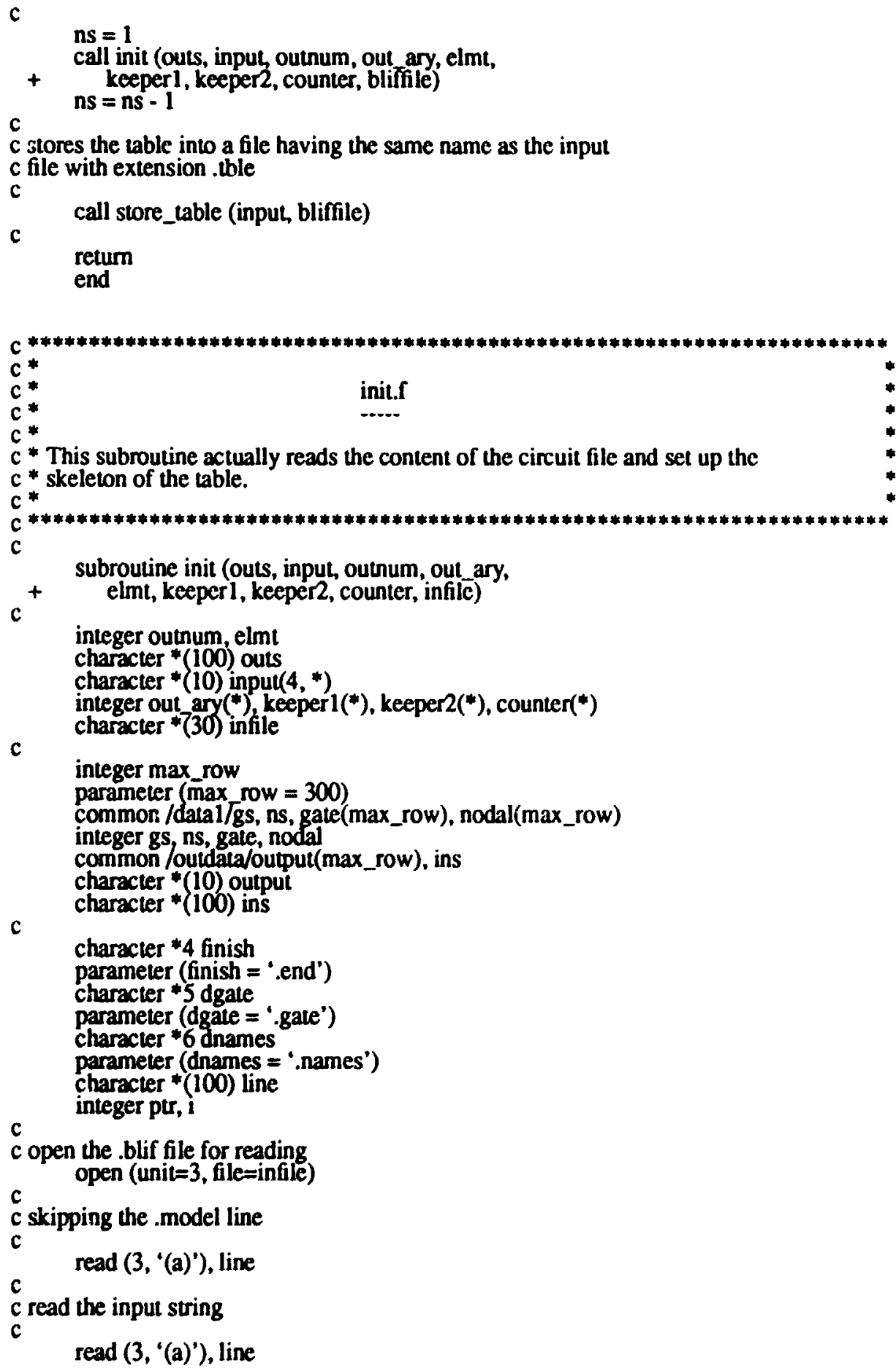




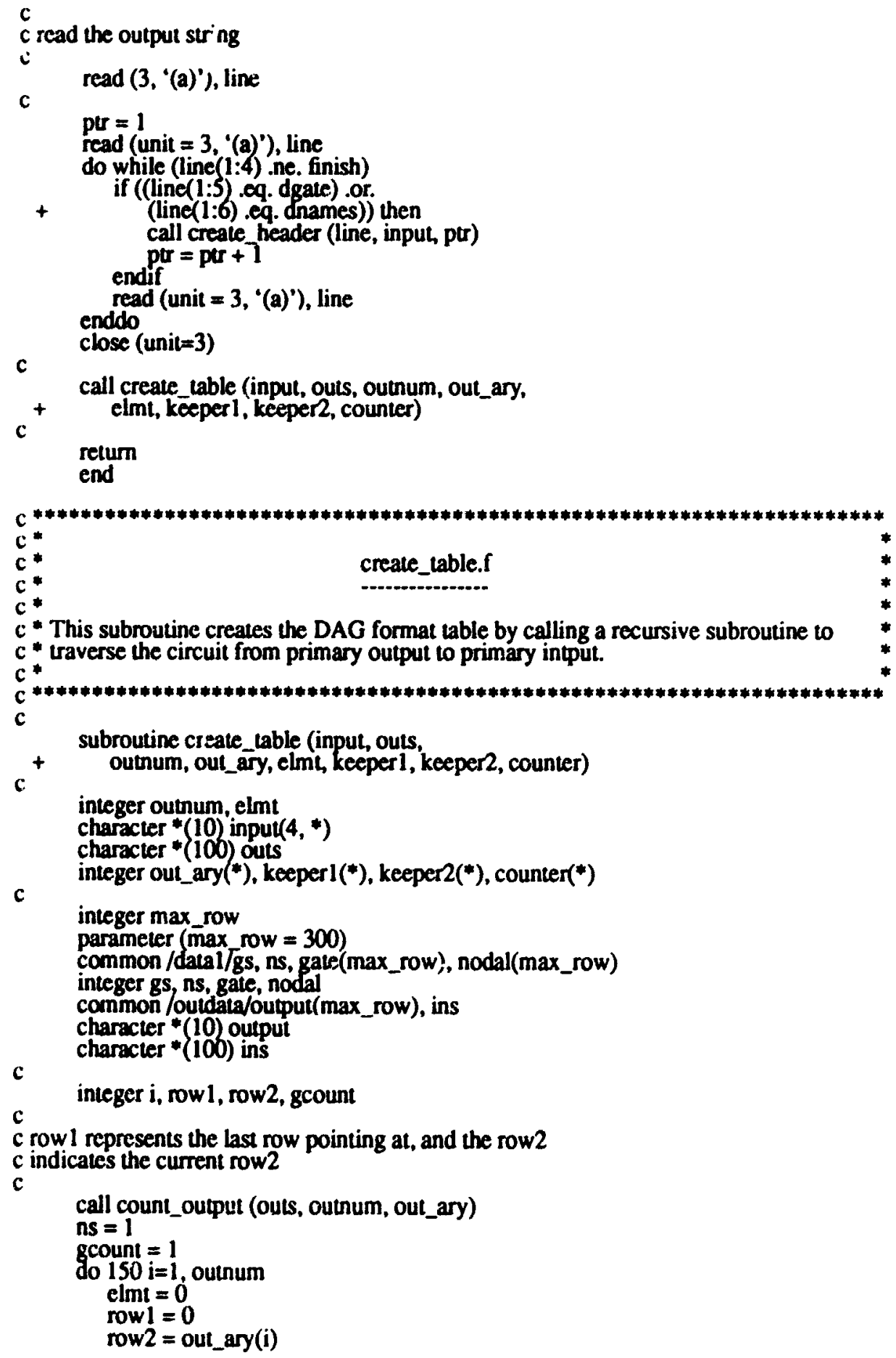


call tracing (gcount, input, outs continue row1, row2, elmt, keeper1, keeper2, counter)

return

end

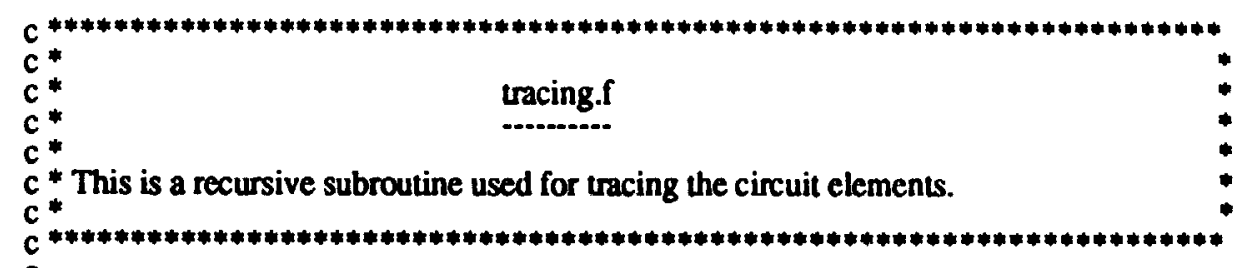

c

$+$

subroutine tracing (gcount, input,

c

outs, row 1, row2, elmt, keeperl, keeper2, counter)

integer gcount, row 1, row2, elmt

character * $(10)$ inpul $(4, *)$

character $*(100)$ outs

$c$

integer keeper $1\left(^{*}\right)$, $\operatorname{keeper} 2\left(^{*}\right)$, counter( $\left(^{*}\right)$

i jieger max_row

Farameter (max row $=300)$

common /datal/gs, ns, gate(max_row), nodal(max_row)

integer gs, ns, gate, nodal

common /outdata/output(max_row), ins

character * $(10)$ output

c

character $*(100)$ ins

character *3 pre

parameter (pre = 'in_')

character * 10 empty.

parameter (empty = " ")

integer $\mathrm{i}$, row 3 , pseudol, pseudo2

c integer get_num

if (input $(1$, row2)(1:3) ne. pre) then nodal (row2) $=$ ns

$\mathrm{ns}=\mathrm{ns}+1$

gate(row2) $=$ gcount

gcount $=$ gcount +1

$i=1$

do while (i.le. 4)

if (input(i, row2) gt. empty) then

if (not. ident (input( $i$, row2), outs)) then

row3 = get_num (input, $i$, row 2 )

if (gate(row3) .eq. 0) then

elmt $=\mathrm{elm} t+1$

keeperl (elmt) = rowl

keeper 2 elmt $=$ row2

counter (elmt) $=\mathrm{i}$

pseudol $=$ row2

pseudo2 $=$ row3

call tracing (gcount, input, outs, pseudol, pseudo2, elmi, keeper 1, keeper 2, counter)

rowl $=\operatorname{keeperl}(\mathrm{elm})$

row2 $=$ keeper 2 (elmt)

$\mathrm{i}=$ counter(elmt) 
double precision ajjtol, pivpct, ffunpr, condu, fpboun

double precision fpdeg, ppstep, redgr (29)

character *8 ramcon(27), ramvar (29), zalpha(56)

integer ncore, nnvars, nfun, maxbas, maxhes, nnobj, nnstop

integer iitlim, ilmser, ipr, ipnA, iipns, iipn6, iiper

integer iiquad, Ideriv, mmodce, inbind(27), nonbas(29), nbind

integer nnonb, inform, lvars (12), mxtabu, ideglm, iiscal

integer nchar, imemcg, ibvbl, iisclg

c character * 76 tuitle

c initialization of LSGRG's parameters

c

data ncore $/ 5000$

data blvar/18*0.0,11*1.20EO/

data buvar $/ 18 * 1 \mathrm{~EB}, 1.5 \mathrm{E} 1,3 * 9.0 \mathrm{E} 0,3 * 1.5 \mathrm{E} 1,3 * 9.0 \mathrm{E} 0,1.5 \mathrm{E} 1 /$

data blcon $/ 27 * 0.0$

data bucon $0,0,1 e 3,0,1 e 3,0,1 e 3,1 e 3,1 e 3,1 e 3$,

$+\quad$ le3, le3, le3, le3, $0,1 \mathrm{e} 3,0,0,1 \mathrm{e}, 1 \mathrm{e} 3$.

+ le3, le3, le3, le3, le3, le3, le3/

data nnvars, nfun, maxbas, maxhes $29,27,27,291$

data lvars/11, 1, 2, 3, 4, 6, 8, 11, 12, 13, 14, 17)

data nnobj 27

data nchar/56/

data defaul $/ 33 * 1.0 /$

c initial set up (should be deleted)

c

data $x x / 18 * 0.0,5.87,4.59,3.35,2.14,1.2,1.2,8.5,7.17,1.2,1.2,1.2 /$

c

double precision prex(29), newx(29), fvalue

c defines polynomials for different gates

c

integer max_cd

parameter ( $\max c d=15$ )

common /f inv/inv_cd(max_cd), a0(max_cd), al(max_cd), a2(max_cd), a3(max_cd)

double precision in $\bar{v}_{-} \mathrm{cd}, \mathrm{a}_{0}, \mathrm{al}, \mathrm{a}, \mathrm{a}$

common /f nand2/nān2_cd (max_cd), bO(max_cd), bl(max_cd), b2(max_cd), b3(max_cd)

double precision nan2 $-\overline{c d}, \mathrm{b0}, \mathrm{bi}, \mathrm{b2}, \mathrm{b3}$

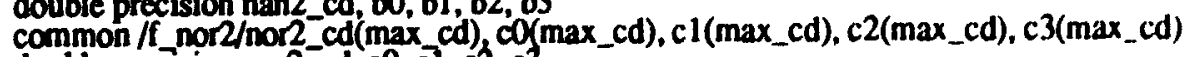

double precision nor $2 \mathrm{~cd}, \mathrm{c0}, \mathrm{cl}, \mathrm{c}^{3}, \mathrm{c}^{3}$

common /f_nand 3/nañ3_cd(max $c d$ ), do(max_cd), d1 (max_cd), d2(max_cd), d3(max_cd)

double precision nan3_cd, do, dI, d2, d3

common /f_nor3/nor3_cd(max_cd) eO(max_cd), el (max_cd), e2(max_cd), c3(max_cd)

double precision nor 3 _cd, eo, el, e2, e3

common /f nand4/nan4 cd (max_cd), fo(max_cd), f1(max_cd), 12(max_cd), 13(max_cd)

double precision nan4_cd, $\mathrm{fo}, \mathrm{fi}, \mathrm{N}, \mathrm{K}$

common /f nor4/nor4_cd(max_cd), gO(max_cd), gl(max_cd), g2(max_cd), g3(max_cd)

c double precision nor4_cd, $80, \overline{\mathrm{g}} 1, \mathrm{~g} 2, \mathrm{~g} 3$

common /pmeter/max, cload

double precision tmax, cload

common /info/nodes(max_row), delay (max_row), actual(max_row)

double precision nodes, delay, actual

common /points/pts

c integer pts

c start of the subroutine

c prompt for variables: Tmax and Cload 


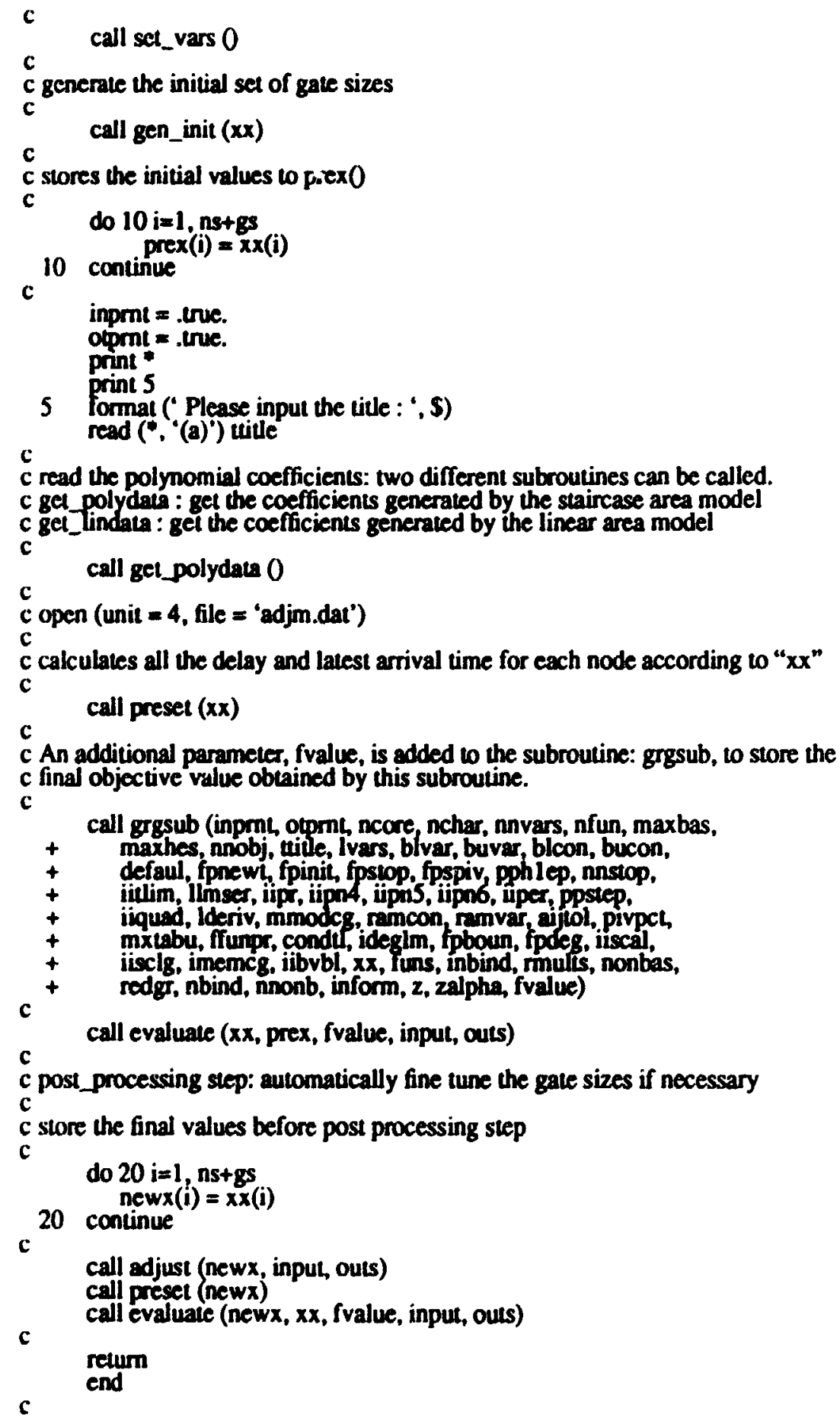




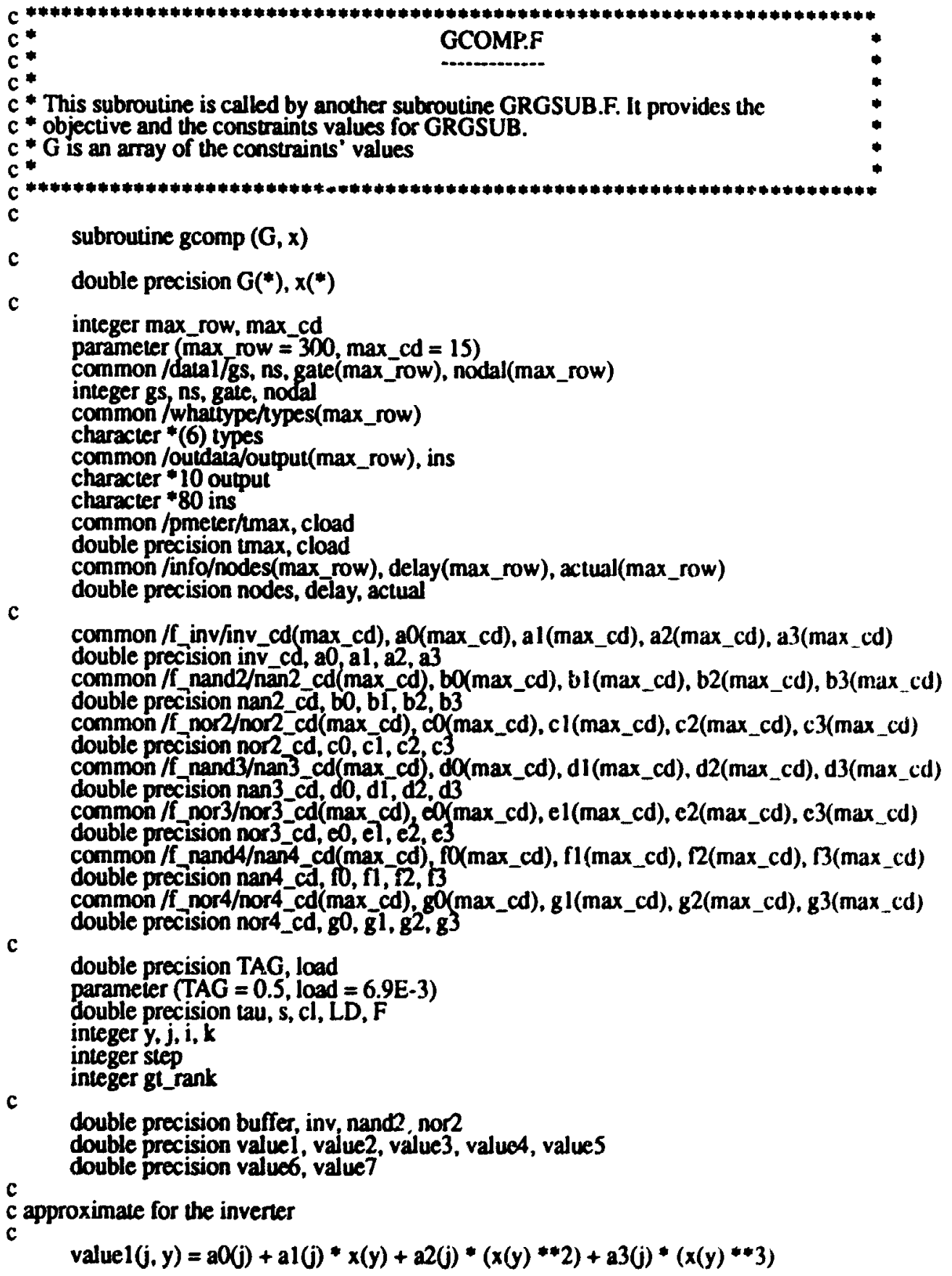




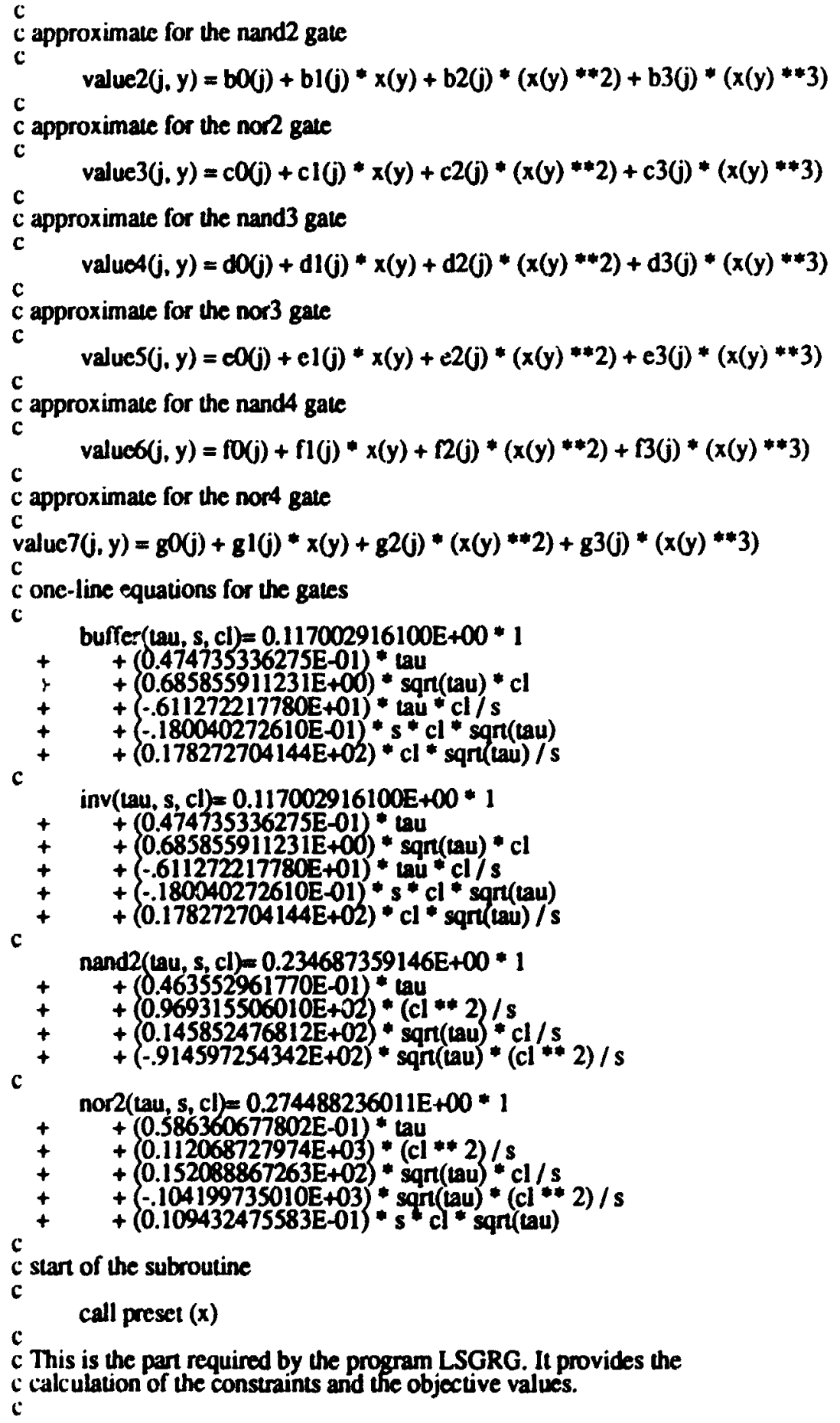




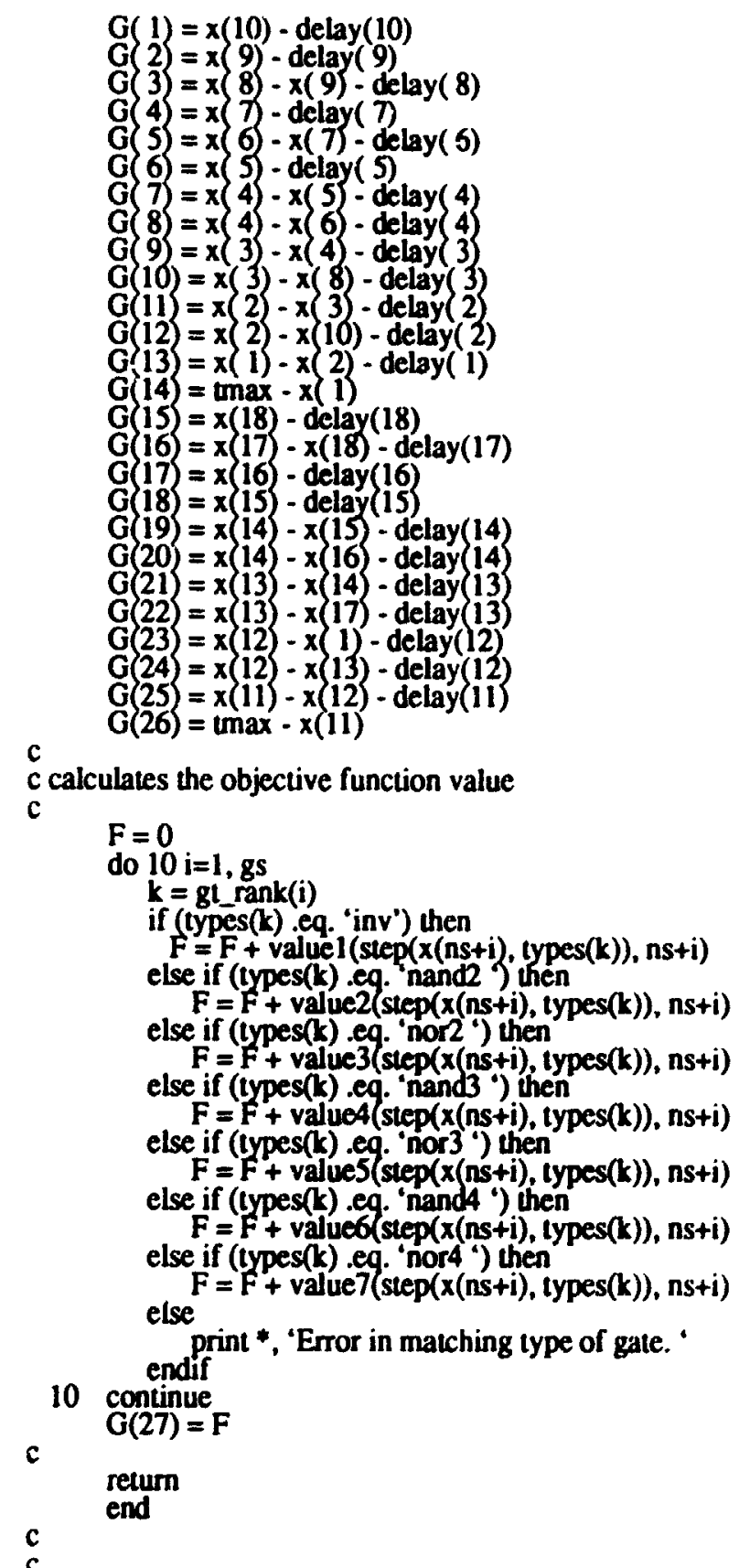

$c$ calculates the objective function value

$\mathrm{c}$$$
F=0
$$$$
\text { do } 10 \mathrm{i}=1, \mathrm{gs}
$$ 
References

\section{References}

(1 | J.M. Shyu, A. Sangiovanni-Vincentelli, J.P. Fishburn, A.E. Dunlop, "Optimization-Based Transistor Sizing", IEEE Journal of Solid-State Circuits, vol.23, no.2, April 1988, pp. 400-409.

[2] P.Penfield and J.Rubinstein, "Signal delay in RC tree networks", Proc. 2nd Caltech VLSI Conf., Mar. 1981, pp. 269-283.

[3] M.C. Chang and C.E. Chen, "PROMPT3 - A Cell-based Transistor Sizing Problem Using Heuristic and Simulated Annealing Algorithms", IEEE 1989 Custom Integrated Circuits Conf., 1989.

[4 ] K.S. Hedlund, "Aesop: A Tool for Automated Transistor Sizing", Proc. of the 24th ACMIIEEE Design Automation Conf., pp. 114-120.

[5 I D. Marple, "Transistor Size Optimization in the Tailor Layout System", Proc. of the 26th A בMIIEEE Design Automation Conf., pp. 4348.

[6 ] D. Luenberger, Linear and Nonlinear Programming, 2nd Ed. AddisonWesley, Massachusetts, 1984.

[7 ] D. Marple, M. Smulders and H. Hegen, "An efficient compactor for 45 degrees layout", Proc. of the 25th Design Automation Conf., June 1988, pp. 396-402.

[8 ] U. Hinsberger and R. Kolla, "Cell Based Performance Optimization of Combinatorial Circuits", Proc. of the EDAC 90', 1990, pp. 594-599.

[9] Mark D. Matson and Lance A. Glasser, "Macromodeling and Optimization of Digital MOS VLSI Circuits", IEEE Transactions on Computer Aided Design, vol. CAD-5, no.4 Oct. 1986, pp. 659-678.

[10] P.E. Gill, W.murray and M.H. Wright, Practical Optimization, Academic Press, London, New York, 1981. 
[11] Michel R.C.M. Berkelaar and Jochen A.G. Jees, "Gate Sizing in MOS Digital Circuits with Linear Programming", Proc. of the IEEE EDAC 90', 1990, pp. 217-221.

[12] Hau-Yung Chen and Santanu Dutta, "A Timing Model for Static CMOS Gates", Proc. of the ICCAD 89', 1989, pp. 72-75.

[13] Shen P. Lin and Malgorzata Marek-Sadowska, "An Accurate and Efticient Delay Model for CMOS Gates in Switching-Level Timing Analysis", Proc. of the IEEE ISCAS 90', 1990, pp. 856-860.

[14] An-Chang Deng, "Piecewise-Linear Timing Delay Modeling for Digital CMOS Circuits", IEEE Transactions on Circuits and Systems, vol. 35 , no. 10, Oct. 88', pp. 1330-1334.

[15] Roger C.F. Shum, "A Timing Macro Model for Performance Optimization of CMOS Logic Circuits", Master thesis in Engineering, 92', Carleton University.

[16] Lucas S.Heusler and Wolfgang Fichtner, "Transistor sizing for large combinatorial digital CMOS circuits", INTEGRATION, the VLSI journal 10 (1991), pp. 155-168.

[17] B. Hoppe, G. Neuendorf, D. Schmitt-Landsiedel and W. Specks, "Optimization of High-Speed CMOS logic circuits with Analytical Models for $\mathrm{Si}_{\iota}$ nal Delay, Chip Area and Dynamic Power Dissipation", IEEE Transactions on Computer-Aided Design, vol. 9, no. 3 , Mar 1990, pp. 236-247.

[18] F.W. Obermeier and R.H. Katz, "An Electrical Optimizer that Considers Physical Layout", Proc. of the 25th ACMIIEEE Design Automation Conf., 1988, pp. 453-459.

[19] H. Youssef, E. Shragowitz and L.C. Bening, "Critical Path Issue in VLSI Designs", Proc. of the ICCAD 89', 1989, pp. 520-523. 
[20] Abadie, J. and Crpentier, J., 1969. "Generalization of the Wolfe Reduced Gradient method to the case of nonlinear constraints", in Fletcher, R. ed., Optimization, Academic Press, New York, 37-47.

[21] P. Gray and C. Searle, Electronic Principles: Physics, Models, and Circuits, New York: Wiley, 1969.

[22] S. Smith and L. Lasdon, "Solving Large Sparse Nonlinear Programs Using GRG", ORSA Journal on Computing, Fall 1991.

[23] M. C Lefebvre, Course notes in VLSI Design, Fall 1990. 

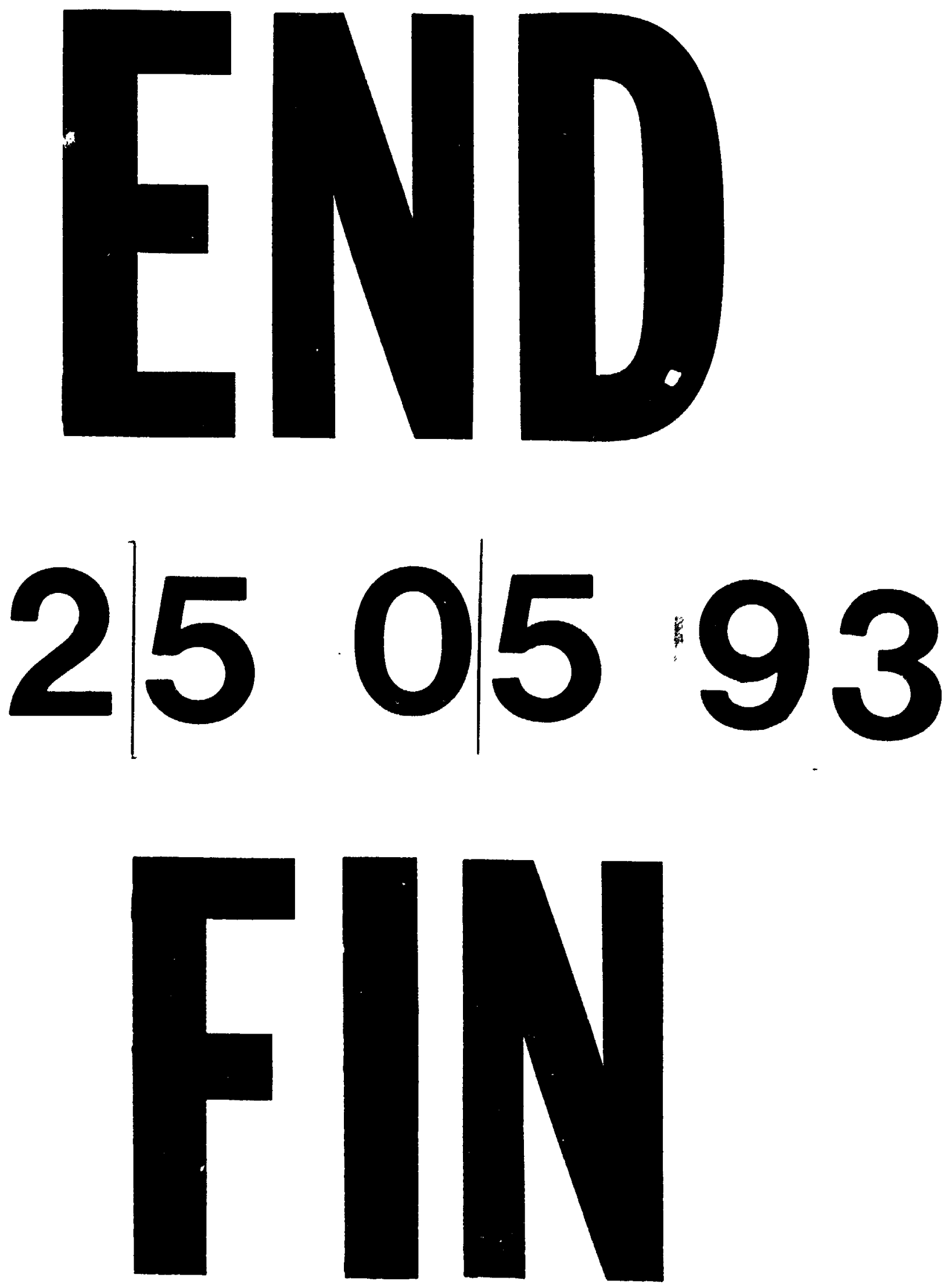